\title{
Updates and Improvements to the Satellite Drag Coefficient Response Surface Modeling Toolkit
}

\author{
Phillip Logan Sheridan \\ West Virginia University, pls0013@mix.wvu.edu
}

Follow this and additional works at: https://researchrepository.wvu.edu/etd

Part of the Astrodynamics Commons, Other Aerospace Engineering Commons, and the Space Vehicles Commons

\section{Recommended Citation}

Sheridan, Phillip Logan, "Updates and Improvements to the Satellite Drag Coefficient Response Surface Modeling Toolkit" (2021). Graduate Theses, Dissertations, and Problem Reports. 8227.

https://researchrepository.wvu.edu/etd/8227

This Thesis is protected by copyright and/or related rights. It has been brought to you by the The Research Repository @ WVU with permission from the rights-holder(s). You are free to use this Thesis in any way that is permitted by the copyright and related rights legislation that applies to your use. For other uses you must obtain permission from the rights-holder(s) directly, unless additional rights are indicated by a Creative Commons license in the record and/ or on the work itself. This Thesis has been accepted for inclusion in WVU Graduate Theses, Dissertations, and Problem Reports collection by an authorized administrator of The Research Repository @ WVU. For more information, please contact researchrepository@mail.wvu.edu. 
Updates and Improvements to the Satellite Drag Coefficient Response Surface Modeling Toolkit

Phillip Logan Sheridan

\author{
Thesis submitted \\ to the Statler College of Engineering and Mineral Resources \\ at West Virginia University \\ in partial fulfillment of the requirements for the degree of \\ Masters of Science in \\ Aerospace Engineering
}

Piyush Mehta, Ph.D., Chair

Jason Gross, Ph.D.

Andrew Rhodes, Ph.D.

Department of Mechanical and Aerospace Engineering

Morgantown, West Virginia

2021

Keywords: Satellite, Drag Coefficient, Gas Surface Interactions, Response Surface Modeling

Copyright 2021 Phillip Logan Sheridan 


\begin{abstract}
Updates and Improvements to the Satellite Drag Coefficient Response Surface Modeling Toolkit
\end{abstract}

\title{
Phillip Logan Sheridan
}

For satellites in Low Earth Orbit, the drag coefficient is a major area of uncertainty. Researchers at the Los Alamos National Laboratory have created a Response Surface Modeling (RSM) toolkit to provide the community with a resource for simulating and modeling satellite drag coefficients in Free Molecular Flow. The toolkit combines the high fidelity of numerical simulation techniques with the speed of regression modeling. Specifically, it uses a training sample of drag coefficients simulated with the Test Particle Monte Carlo method with the robust Gaussian Process Regression approach. The RSM toolkit is the prime process to become a toolkit of other expanded capabilities. In this work, the capabilities are specifically expanded to include uncertainty quantification, utilizing Python, to create a free and open source software, and adapting the toolkit for automatic development of regression models of satellites with rotating components. The new RSM toolkit has been developed to have user friendly installation along with extensive documentation of operation. 


\section{ACKNOWLEDGMENTS}

I would like to thank my family for all their support through my academic career. I would also like to thank my advisor and committee chair, Dr. Piyush M. Mehta. His advice and teachings as well as all his patience and support have lead me to a successful graduate education. Furthermore, I would like to thank my cohort members and colleagues for all their help as well as my committee members for all their teachings and guidance.

A very notable thanks to Dr. Guillermo Avendo-Franco, with whom I would not have been able to mold my research into a reputable software. His teachings and support have helped my engineering capabilities go above and beyond. Thanks to Dr. Smriti Paul as well. His assistance with this software was invaluable and his patience was unyielding. I would also like to thank Dr. Andrew C. Walker of Los Alamos National Laboratory. His research and creation of the TPMC code was the basis for my research. Furthermore, his assistance in the development of this new software was pinnacle.

Thanks to the West Virginia University super-computing systems, which are funded in part by the National Science Foundation EPSCoR Research Infrastructure Improvement Cooperative Agreement \#1003907, National Science Foundation Major Research Instrumentation Program (MRI) Award \#1726534, the state of West Virginia (WVEPSCoR via the Higher Education Policy Commission) and West Virginia University. 


\section{CONTENTS}

1. Introduction 1

1.1. Motivation . . . . . . . . . . . . . . . . 1

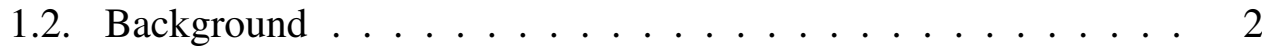

1.2.1. Satellite Drag Theory . . . . . . . . . . . . . . . 2

1.2.2. Satellite Drag Coefficient. . . . . . . . . . . . . . . 2

1.2.3. Gas-Surface Interactions . . . . . . . . . . . . . . 3

1.2.4. Total Drag Coefficient . . . . . . . . . . . . . . . 7

1.2.5. Computational Analysis Methods . . . . . . . . . . 9

2. Los Alamos National Laboratory RSM Toolkit 13

2.1. Stereolithography(STL) Files . . . . . . . . . . . . . . . . 14

2.2. Overview . . . . . . . . . . . . . . . . 15

2.3. Automated RSM . . . . . . . . . . . . . . . . 17

2.3.1. LANL Regression Model . . . . . . . . . . . . . . . 20

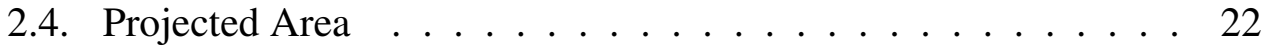

$2.5 . \quad$ Model Evaluation . . . . . . . . . . . . . . . . . . . . . . . . 24

2.6. Limitations of LANL RSM Toolkit . . . . . . . . . . . . . . 25

3. West Virginia University's RSM Software Package 26

3.1. Extension to Complex Geometries . . . . . . . . . . . . . . 28

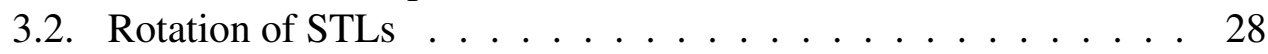

3.3. Check Water-Tightness . . . . . . . . . . . . . . . 31

3.4. WVU Regression Model $\ldots \ldots \ldots$. . . . . . . . . . . 32

3.4.1. Uncertainty . . . . . . . . . . . . . . . . . 33

3.5. Model Evaluation . . . . . . . . . . . . . . . . . . . . . 34

3.6. Compilation/Installation of Code . . . . . . . . . . . . 34

3.6.1. Modules Required . . . . . . . . . . . . . . . . . 35

3.6.2. Compilation . . . . . . . . . . . . . . 36

3.7. Navigation. . . . . . . . . . . . . . . . . . . . . . . . . . . 38

3.7.1. Overview . . . . . . . . . . . . . . . . . . 38

$3.7 .2 . \quad$ Inputs $\ldots \ldots \ldots \ldots$. . . . . . . . . . . . . 39

3.7.3. Outputs . . . . . . . . . . . . . . . . . . . . 40 40

3.7.4. RSM_TPMC . . . . . . . . . . . . . . . 41

3.8. Simulation Execution . . . . . . . . . . . . . . . . . 42

3.8.1. Simulation Inputs . . . . . . . . . . . . . . . . . 42

3.8.2. STL_Files . . . . . . . . . . . . . . . . 45

3.8.3. STL_Rotation_Inputs. . . . . . . . . . . . . . 45

3.9. RSM Code Execution . . . . . . . . . . . . . . . . . . . 47

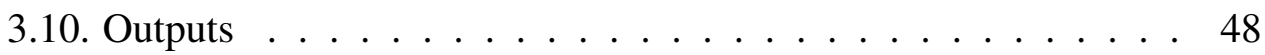

3.10.1. TPMC Simulation Outputs . . . . . . . . . . . . . . 48

3.10.2. Regression Model $\ldots \ldots \ldots$. . . . . . . . . . . . 49 
3.10.3. Model Evaluation Outputs . . . . . . . . . . . . 50

3.10.4. Latin Hypercube Sampling . . . . . . . . . . . . 50

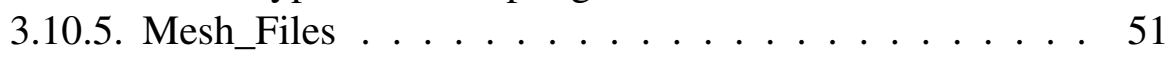

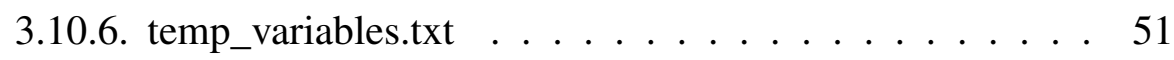

3.11. Model Evaluation . . . . . . . . . . . . . . 52

3.11.1. Model Evaluation Inputs . . . . . . . . . . . . . . . 52

3.11.2. Execution of Model Evaluation . . . . . . . . . 56

3.11.3. Outputs of Model Evaluation . . . . . . . . . . 56

4. Results 56

4.1. Check Water-Tightness . . . . . . . . . . . . . . 56

4.2. Comparison of Closed Form Solutions . . . . . . . . . . . . . 58

4.3. Gaussian Process Regression . . . . . . . . . . . . . . . . . . 64

4.3.1. GPR Prediction Results . . . . . . . . . . . . . . 64

4.3.2. Model Calibration .............. . . 69

4.3.3. Computational Analysis Comparison . . . . . . . . . 71

4.3.4. Run Time Comparison . . . . . . . . . . . . . . . 74

4.4. Documentation .................... . . 75

5. Conclusion/Future Work 75

5.1. Conclusion . . . . . . . . . . . . . 76

5.2. Future Work . . . . . . . . . . . . . . 76

\begin{tabular}{ll}
\hline Appendices & 78
\end{tabular}

A. Example of WVU RSM Toolkit Operation with Rotation 78

A.1. Creating an STL File . . . . . . . . . . . . . . . 79

A.2. Example Inputs . . . . . . . . . . . . . . . . . . . . . . . . . . . . . . . 82

A.3. Example Execution . . . . . . . . . . . . . . . . . 84

A.4. Example Outputs . . . . . . . . . . . . . . . . . . 85

A.5. Example Model Evaluation . . . . . . . . . . . . . . . 86

B. Code Resources 88

B.1. Code . . . . . . . . . . . . . . . . . 88

B.2. Developers ...................... 88 


\section{LIST OF FIGURES}

1.1. Reflections of different Gas-Surface Interactions . . . . . . . . . 4

1.2. Surface coverage of atomic oxygen using a mass spectrometer . 5

1.3. Classification of Flow Regimes based on Knudsen number [2] . 10

2.1. Example STL File, Slice of a Cube . . . . . . . . . . . . . 15

2.2. Flowchart of LANL RSM toolkit Operation . . . . . . . . . . . 16

2.3. Top level Directory of LANL RSM Toolkit . . . . . . . . . . . 17

2.4. Automated_RSM folder of LANL RSM Toolkit . . . . . . . . . 18

2.5. tpm.inp input file for tpm.c and Automated_RSM . . . . . . . . 20

2.6. RSM_Area folder of LANL RSM Toolkit . . . . . . . . . . . 23

2.7. RSM_Cd folder of LANL RSM Toolkit . . . . . . . . . . . . . 24

2.8. RSM.inp input file for RSM.c. . . . . . . . . . . . . . . . 25

3.1. Flowchart of WVU RSM Toolkit Operation . . . . . . . . . . 27

3.2. Top Level Directory of WVU RSM Toolkit . . . . . . . . . . . 38

3.3. Inputs Directory of WVU RSM Toolkit . . . . . . . . . . . 39

3.4. Outputs Directory of WVU RSM Toolkit. . . . . . . . . . . . . 40

3.5. RSM_TPMC Directory of WVU RSM Toolkit. . . . . . . . . . 41

3.6. Example of the Simulation.json file. . . . . . . . . . . . . 43

3.7. Correlation between STL_Rotation_Input Files . . . . . . . . . 46

3.8. Example of the parent.txt file . . . . . . . . . . . . 46

3.9. Example of the hinge_points1.txt file. . . . . . . . . . . . . . . . . . . . . . 47

3.10. Example of the hinge_points2.txt file . . . . . . . . . . . . 47

3.11. Visualization of Model Performance . . . . . . . . . . . . . . . 49

3.12. Example of the Model_Evaluation_Inputs.json file . . . . . . 53

3.13. Code from model_evaluation_script.py, calculation of the fractional surface coverage of adsorbed oxygen with different ADS models. ..................... 54

$4.1 . \quad$ CWT Results . . . . . . . . . . . . . . . . . . 57

4.2. Closed Form Solution of a Cube with Varying Velocity . . . . . 59

4.3. Closed Form Solution of a Cube with Varying Atmospheric Temperature .................... 60

4.4. Closed Form Solution of a Sphere with Varying Velocity . . . . 61

4.5. Closed Form Solution of a Sphere with Varying Atmospheric

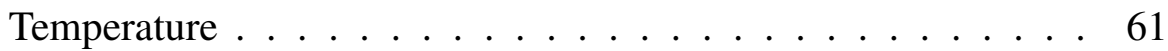

4.6. Closed Form Solution of a Sphere with Varying Atmospheric Temperature ..................... 62

4.7. Closed Form Solution of a Cylinder with Varying Velocity . . . 63

4.8. Closed Form Solution of a Cylinder with Varying Atmospheric Temperature ................. 63

4.9. Closed Form Solution of a Cylinder with Varying Atmospheric Temperature ..................... 64

4.10. GPR Results . . . . . . . . . . . . . . . . . . . . 65 
4.10. GPR Results . . . . . . . . . . . . . . . . . . 66

4.10. GPR Results . . . . . . . . . . . . . . . . . . . . . 67

4.11. Satellite with rotating solar panels . . . . . . . . . . . . . 67

4.12. GPR Results . . . . . . . . . . . . . . . . . . . . . . . . . 68

4.12. GPR Results . . . . . . . . . . . . . . . . . . . . . . . . . . 69

4.13. Consistency Plots for Sphere and Champ for Drag Coefficient Prediction Using Gaussian Process Regression for the Test Dataset 71

4.14. CHAMP Satellite . . . . . . . . . . . . 72

4.15. Comparison of GPR versus known values . . . . . . . . . . 73

A.1. Structure and Mesh of example CubeSat with Solar Panels . . . 78

A.2. Body Component of Example CubeSat . . . . . . . . . . . . . 79

A.3. Left Solar Panel Component of Example CubeSat . . . . . . . . 80

A.4. Right Solar Panel Component of Example CubeSat . . . . . . . 80

A.5. Mesh for Body Component of Example CubeSat, GMSH . . . . 81

A.6. Simulation Input for Example . . . . . . . . . . . . . . . . . . . 82

A.7. Example Rotation Inputs . . . . . . . . . . . . . . . . . 83

A.8. Simulation Input for Example . . . . . . . . . . . . . . . 83

A.9. Rotation Direction for Example. . . . . . . . . . . . . . . . . . 84

A.10.RSM Logo and Initial Output . . . . . . . . . . . . . . . . . . 85

A.11.Thirty Files for the Example Regression Model . . . . . . . . . 85

A.12.Example Model_Evaluation.json file . . . . . . . . . . . 86

A.13.Example CSV file . . . . . . . . . . . . . . . 86

A.14.Example Results . . . . . . . . . . . . . . . 87 


\section{ACRONYMS}

ASCII American Standard Code For Information Interchange. 13

ASSIST Astrodynamics, Space Science, and Space Technology. 32

CAD Computer-Aided Design. 14

CFD Computational Fluid Dynamics. 11

CLL Cergani-Lampis-Lord. 7

DAC DSMC Analysis Code. 11

DRIA Diffuse Reflection with Incomplete Accommodation. 7

DSMC Direct Simulation Monte Carlo. 10

FCC Federal Communications Commission. 1

FMF Free Molecular Flow. 9

GPR Gaussian Process Regression. 13

GSI Gas-Surface Interaction. 3

GSL GNU Scientific Library. 35

IEEE Institute of Electrical and Electronics Engineers. 1

LANL Los Alamos National Laboratory. 13

LEO Low Earth Orbit. 1

LHS Latin Hypercube Sampling. 18

MPI Message Passing Interface. 11

RSM Response Surface Modeling. 5

STL Stereolithography. 13, 14

TPMC Test Particle Monte Carlo. 10

WVU West Virginia University. 13 


\section{INTRODUCTION}

\subsection{Motivation}

Expeditious growth in space related technologies have caused an immense increase in demand for satellites in both the private and public sector. According to Institute of Electrical and Electronics Engineers (IEEE), the Federal Communications Commission (FCC) has approved SpaceX to launch a total of nearly 12,800 satellites for its Starlink constellation. Already to date, SpaceX has launched 1,300 of these satellites [35]. Constellations for internet and other consumer services are becoming more and more popular. Other constellations like OneWeb, Telesat, and Project Kuiper are also planning to send satellites en masse to orbit. Between these four companies, 46,100 satellites are planning to be launched in the next decade [4]. With so many satellites in orbit, it is imperative that operators have a firm understanding of where their assets will be at any given time. Furthermore, NASA states that as of 2021 there are more than 27,000 pieces of orbital debris, or "space junk," that are being tracked by the Department of Defense's global Space Surveillance Network [27]. Most of these satellites and debris are going to, or already do, reside in Low Earth Orbit (LEO). In LEO, drag is the dominant source of orbit position error resulting from inaccurate drag coefficient and density modeling. Improved modeling will ultimately help to avoid satellite-satellite and satellite-debris collisions which can cause millions of dollars in damage and the creation of more space debris. The work presented here is targeted at improved modeling of satellite drag coefficient. 


\subsection{BACKGROUND}

\subsubsection{SATEllite Drag THEORY}

The theoretical model for calculating satellite drag is given by

$$
\vec{a}_{d r a g}=\frac{1}{2} \rho \frac{\left(C_{D} A\right)}{m v_{r e l}^{2}} \frac{\vec{v}_{r e l}}{\left|\vec{v}_{r e l}\right|}
$$

where $\vec{a}_{d r a g}$ is the acceleration due to drag, $\rho$ is the atmospheric density, $C_{D}$ is the drag coefficient, $A$ is the projected area of the satellite as seen by the flow, $m$ is the mass of the satellite, and $\vec{v}_{r e l}$ is the velocity of the satellite with respect to the corotating atmosphere. Error in calculating the acceleration due to drag comes from two main sources: density and drag coefficient. An important group of variables also worth noting is $C_{D} \frac{A}{m}$. Together, these variables make up the Ballistic Coefficient. The Ballistic Coefficient wraps the uncertainty of the satellite specific variables into a singular variable of uncertainty.

\subsubsection{Satellite DRAG CoefFicient}

In the context of orbital dynamics, the drag coefficient $\left(C_{D}\right)$ is considered in three distinct ways: a physical $C_{D}$, a fitted $C_{D}$, and a fixed $C_{D}$ [25]. A fixed $C_{D}$ is a constant value. The fitted $C_{D}$ is estimated as part of an orbit determination process. It is specific to the atmospheric model used and includes the limitations of the atmospheric model and also frequently absorbs other force model errors. Physical $C_{D}$ is determined by the exchange of energy and momentum of the freestream atmospheric molecules with the spacecraft surface [33]. Throughout this work, the term $C_{D}$ will refer to a physical $C_{D}$. 


\subsubsection{GAS-SURFACE INTERACTIONS}

Gas-Surface Interaction (GSI) models the exchange of energy and momentum between atmospheric molecules and the surface of an object. The energy exchange is represented by adsorption modeling while the momentum exchange is represented in reflection modeling. Three accommodation coefficients are used to define a GSI model; the normal and tangential momentum accommodation coefficients, $\sigma_{n}$ and $\sigma_{t}$, and the energy accommodation coefficient $\alpha$. The energy accommodation coefficient is defined by the following.

$$
\alpha=\frac{E_{i}-E_{r}}{E_{i}-E_{s}}
$$

$E_{i}$ is the kinetic energy of an incident molecule, $E_{r}$ is the kinetic energy of a reflected molecule, and $E_{s}$ is the energy that a molecule would have after being re-emitted in thermal equilibrium with the surface. The other two coefficients define how the particle is reflected from the surface. If the tangential and normal momentum coefficients are equal to one, then it is said that the deflection is diffuse. If both coefficients are equal to zero then it is said the deflection is specular. Any other values of $\sigma_{n}$ and $\sigma_{t}$ are said to be quasi-specular [37]. Figure 1.1 is an illustration of the different reflections. 

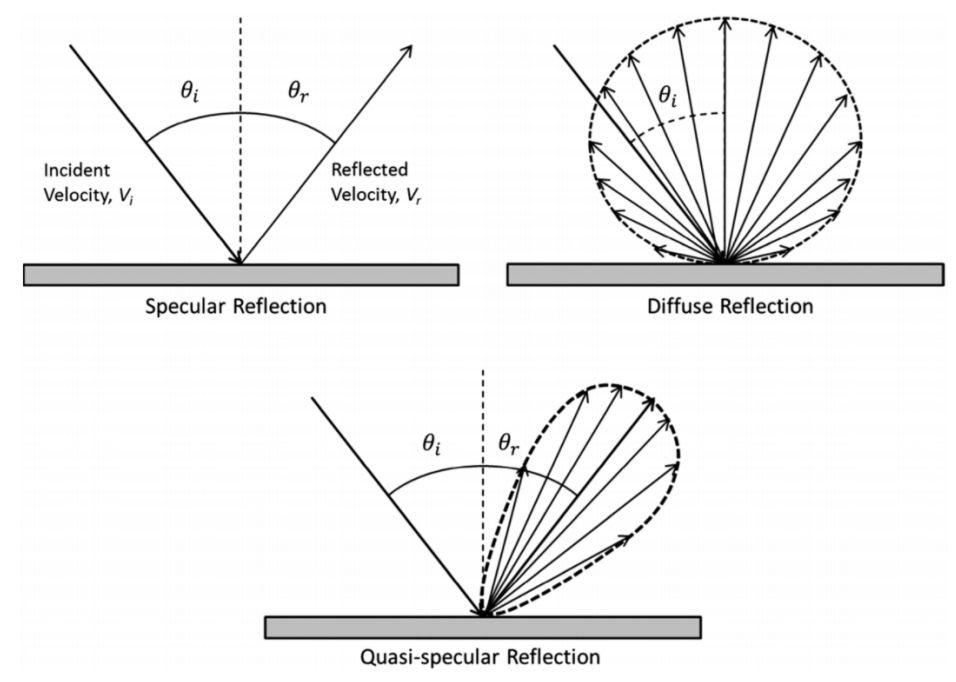

Figure 1.1: Reflections of different Gas-Surface Interactions [37]

Together, these define the GSI modeling. Several GSI models have been developed to simulate these types of Gas-Surface Interactions including Maxwell's Model, Cergani-Lampis-Lord, and Diffuse Reflection with Incomplete Accommodation.

1.2.3.1. AdSORPTION MODELS Laboratory experiments began in the early 1900's to measure the reflection of molecules from surfaces. But, since the 1960's, satellite experiments have been performed using pressure gauges and mass spectrometers to reveal that satellite surfaces are covered by adsorbed gases which can vary given different altitudes [26]. The following figure shows the data from the mass spectrometer on board NASA's OGO-6 satellite. 


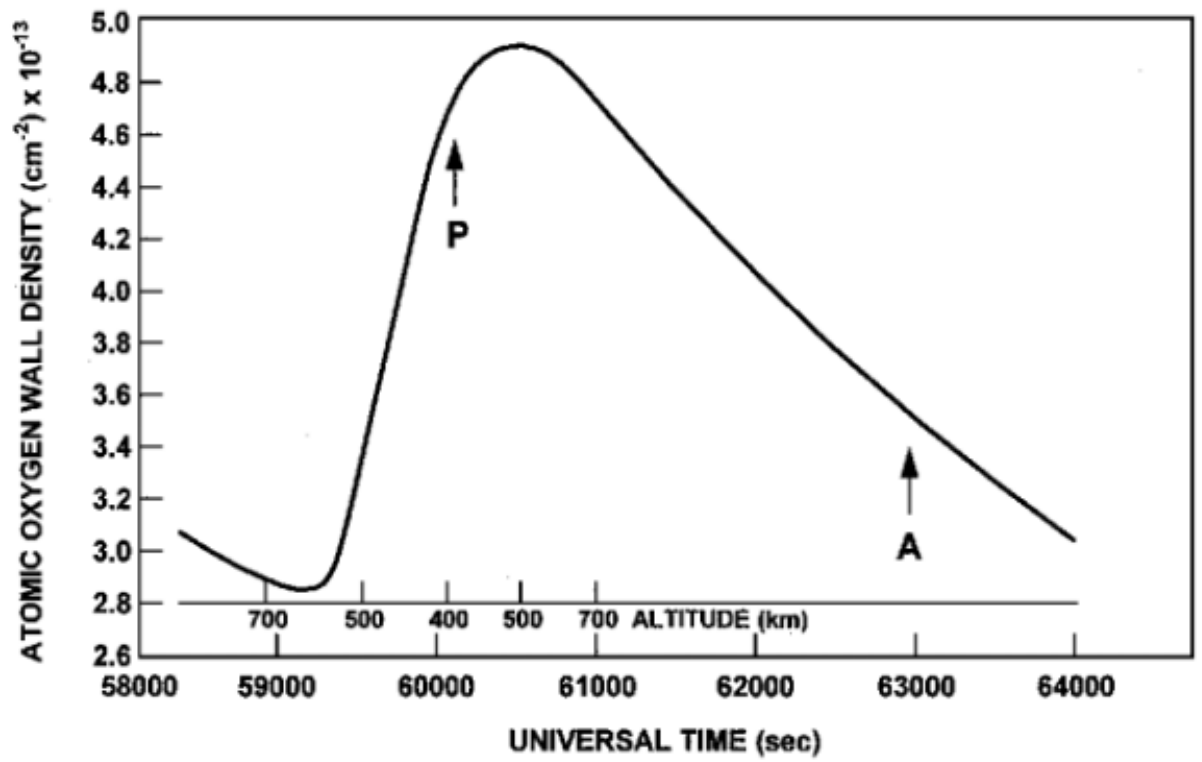

Figure 1.2: Surface coverage of atomic oxygen using a mass spectrometer [24]

The surface coverage of oxygen is much higher at perigee $(\mathrm{P})$ than at apogee (A). This shows that adsorption occurs at lower altitudes and desorption occurs at higher altitudes [26]. This realization has led to the necessity of creating different adsorption models of oxygen.

An adsorption isotherm is an empirical representation of the phenomenon governing the retention, release, or mobility of a substance from a fluid medium to a solid at a constant temperature. To date, there have been a total of fifteen isotherms developed: Langmuir, Freundlich, Brunauer-Emmett-Teller, Redlich-Peterson, Dubinin-Radushkevich, Temkin, Toth, Koble-Corrigan, Sips, Khan, Hill, Flory-Huggins, MacMillan-Teller, Frenkel-Halsey-Hill, and Radke-Prausnitz isotherm [7]. Two of the most notable are the Freundlich and Langmuir isotherms, both are used in the development of the Response Surface Modeling (RSM) software outlined in this work.

The Freundlich isotherm is the first adsorption isotherm created in 1906 by 
Herbert Freundlich [8]. It is a robust empirical model that can be applied to multi-layer adsorption, with non-uniform distribution of adsorption heat and affinities over a heterogeneous surface [7]. The following equation is the empirical model of the Freundlich isotherm.

$$
\frac{x}{m}=k P^{\frac{1}{n}}
$$

$x$ is the mass of adsorbate adsorbed, $m$ is the mass of the adsorbent, $P$ is the pressure of adsorbate, and $k$ and $n$ are empirical constants for each adsorbent-adsorbate pair at a given temperature

The Langmuir Isotherm was created by Irving Langmuir in 1916 [20]. In its formulation is an empirical model that assumes mono-layer adsorption, with adsorption that can only occur at a finite number of definite localized sites, that are identical and equivalent, with no lateral interaction and steric hindrance between the adsorbed molecules, even on adjacent sites [7]. The Langmuir isotherm equation is defined as the following.

$$
\theta=\frac{K P_{o}}{1+K P_{o}}
$$

$\theta$ is the fractional surface coverage, $K$ is the Langmuir adsorbate constant, and $P_{O}$ is the partial pressure of atomic oxygen.

\subsubsection{MAXWELL's MODEL Maxwell's model assumes that across the dis-} tribution of reflected particles, those that are specularly reflected are decomposed into a fraction, $\epsilon$, where the remainder are assumed to be diffusely reflected. Molecular beam experiments [15-18] have shown that particles are not reflected according to Maxwell's model but are instead reflected from a surface 
in a quasi-specular manner.

\subsubsection{CERGANI-LAMPIS-LORD Cergani-Lampis-Lord (CLL) is a GSI that} treats the normal and tangential momentum coefficients independently. It was originally derived by Cergani and Lampis in 1971 by using scattering kernels that describe the relationship between the incident and reflected velocity distribution functions when particles interact with a solid surface [3]. In 1989 R.G. Lord extended the CL model to the cases of diffuse reflection with incomplete accommodation and internal energy accommodation [21], thus creating the Cergani-Lampis-Lord GSI model.

\subsubsection{Diffuse Reflection InComplete Accommodation In the Dif-} fuse Reflection with Incomplete Accommodation (DRIA) GSI model, particles are always reflected with a diffuse angular distribution, based on Knudsen's cosine law [19], but may exchange energy with the surface depending on the value of the energy accommodation coefficient. When interacting with a contaminated surface, molecules adsorb to the layer of atomic oxygen becoming fully accommodated and are reflected with a diffuse distribution [38].

\subsubsection{Total DRAG COEFFICIENT}

A self-consistent quasi-specular drag coefficient model requires relating the effective energy accommodation back to the atmospheric properties [37]. Such a model was developed by Walker et al. in 2014 [37]. Pilinski et al. showed that the variation of the energy accommodation coefficient assuming diffuse reflection is well matched by a Langmuir isotherm dependent on the partial pressure of atomic oxygen, $P_{O}$ [29]. Later work by Pilinski et al. [28, 30] was the first to 
quantitatively test the validity of the Langmuir isotherm model for LEO satellites. The model developed by Walker et al. builds on the work of Pilinski et al. [29] but uses a modified approach with a different scattering kernel and fitting technique. The Walker et al. approach fits a Langmuir isotherm, not to the effective energy accommodation coefficient, but instead to the fraction of the surface covered by atomic oxygen, $\theta$. Modeling the mixed reflection properties of surfaces with impurities such as adsorbed atomic oxygen through $\theta$ was previously suggested by Goodman [12]. The Langmuir isotherm can only model monolayer adsorption; however, $P_{O}$ at the altitudes of interest for LEO satellites is well below the threshold where only monolayer adsorption can occur. Many other adsorption models exist, but the Langmuir isotherm has been used extensively to model the adsorption of atomic oxygen to satellite surfaces [14, 23, 29]. Thus the total drag coefficient can be found using the following equation.

$$
C_{D, T}=(1-\theta) C_{D, s}+\theta C_{D, a d s}
$$

$C_{D, T}$ is the total drag coefficient of the satellite, $C_{D, s}$ is the surface drag coefficient based on a clean satellite surface, and $C_{D, a d s}$ is the drag coefficient of a the surface completely covered by an adsorbate, atomic oxygen in this case. The linear relation between $C_{D}$ and $\theta$ assumes that the adsorbed atomic oxygen uniformly covers the satellite surface.

$C_{D, a d s}$ is computed using the diffuse reflection with complete accommodation, $\alpha=1 . C_{D, s}$ is computed based on Goodman's empirical model [11] for the energy accommodation coefficient.

$$
\alpha_{s}=\frac{3.0 \mu}{\left(1+\mu^{2}\right)}
$$


$\alpha_{s}$ is the energy accommodation coefficient for the gas particles of the clean satellite surface, and $\mu$ is the ratio of the average mass of the atmospheric gases to the particles that compose the satellite surface. The particles that compose the satellite surface mass are dependent on the material and finish of the satellite surface.

\subsubsection{Computational Analysis Methods}

1.2.5.1. FLOW REGIMES In order to determine the appropriate computational method for modeling in a flow, the Knudsen number is used. This nondimensional parameter defines the flow regime the analysis is occurring in. It is defined by the following equation where $\lambda$ is the mean free path and $\mathrm{L}$ is a characteristic length of the object.

$$
K n=\frac{\lambda}{L}
$$

The Free Molecular Flow (FMF) regime is recognized as a flow with a Knudsen number greater than $\sim 10$, typically occurring at $\sim 150 \mathrm{~km}$ altitude and above. This regime is where the interest in modeling satellites takes place. This type of flow is considered to occur in ultra-high vacuum conditions where intermolecular collisions are considered to be nonexistent and GSIs are the dominant phenomena. 


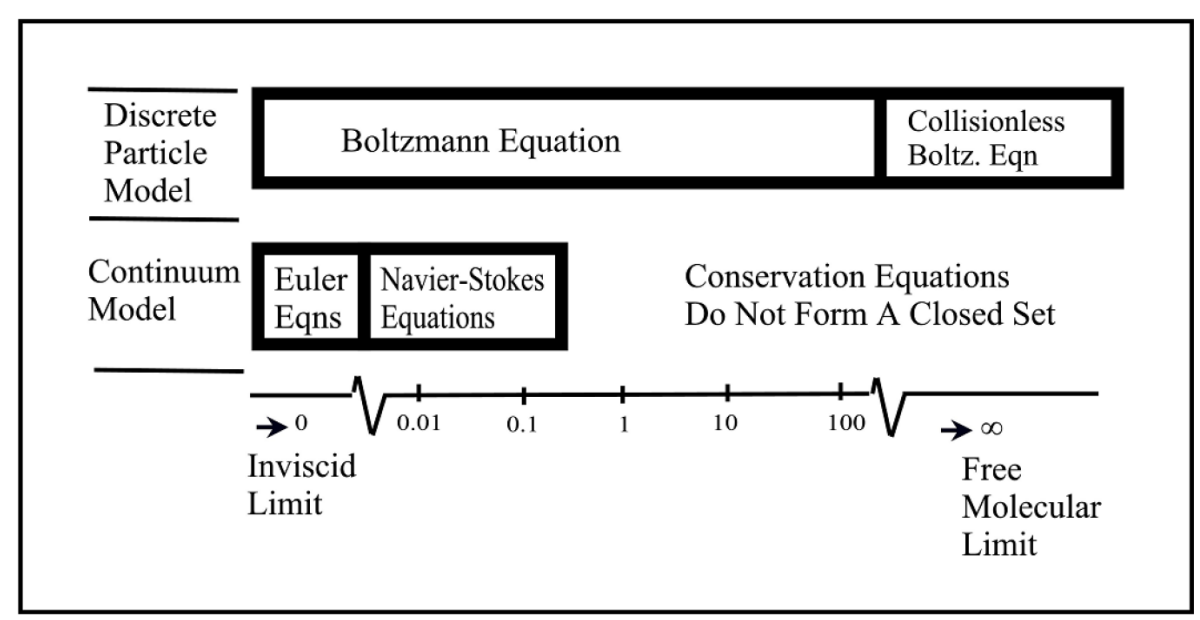

Figure 1.3: Classification of Flow Regimes based on Knudsen number [2]

Currently, there are several computational analysis methods for modeling the drag on satellites. First, there are closed-form solutions of $C_{D}$ for simple geometries (e.g. cube, cylinder, sphere, etc.) in the FMF regime; however, most satellites do not have simple geometries. The most common computational tools used for computing $C_{D}$ in FMF are the Test Particle Monte Carlo (TPMC) method and the Direct Simulation Monte Carlo (DSMC) method. The Monte Carlo technique is a popular method and has been used in the past for various studies to obtain normalized aerodynamic force coefficients. A fundamental difference between the two is that DSMC simulates particle collisions and TPMC does not.

1.2.5.2. DSMC DSMC was developed and applied by Graeme Bird in 1963 [1]. DSMC is widely used for its high fidelity modeling of flow in transitional flow regimes where the assumptions of FMF no longer hold true. The ability to simulate particle collisions is a valuable tool, but it can cause simulations to be computationally expensive.

The panel method is used for DSMC simulations. With this method, the struc- 
ture of the satellite is made into facets. Each facet is an area on the object where properties are defined and calculated. The fidelity of the model is dependent on the mesh, similarly to most CFD software.

DSMC operates by inserting molecules into a flow field. To do this, the code either performs initial creation of molecules at the first time step, a surface flux from the boundaries, or uses the reservoir method. In the reservoir method, the DSMC simulations are extended to an external region adjacent to the DSMC domain. Particles with the desired velocity distribution are generated in the reservoir at every time step and those that enter the DSMC domain are accepted while the remaining particles are rejected. In DSMC simulations, like other atmospheric CFD, the computational domain is part of a larger flow environment. Because the computational domain is part of the larger flow environment, the boundary conditions are often set to be freestream conditions where molecules are allowed to leave and enter the computational domain and the number of simulated molecules varies with time. Every simulated molecule in DSMC represents a number of molecules of real gas. This number acts as the statistical weight of a simulated molecule as well.

NASA has created the DSMC Analysis Code (DAC) to study rarefied gas dynamics pertaining to problems such as atmospheric re-entry. This code is an export controlled item that is only accessible to U.S. persons. DAC has the ability to automatically adapt the collision grid to resolve the local mean free path of a flow field. DAC also utilizes a pre-processor which specifies the time step and statistical weight for representative molecules given the gas conditions such as number density and freestream velocity. Furthermore, DAC offers the ability to perform computations in parallel using MPI. DAC is capable of simulating multiple GSIs including Maxwell's Model, CLL, and DRIA. These features 
make DAC a powerful tool; however, it is more computationally expensive than TPMC and therefore not ideal for FMF where inner-molecular collisions are negligible.

1.2.5.3. TPMC Test Particle Monte Carlo is a Monte Carlo simulation technique first proposed by D. H. Davis circa 1961 [5]. TPMC is an excellent modeling tool as it is computationally inexpensive and as high fidelity as other known models in the free molecular flow regime. A user can model the satellite with pronounced fidelity, often with an error less than $1.0 \%$ [22].

TPMC works similarly to DSMC where molecules, or "test particles" in this case, are represented by a number of molecules of real gas. These test particles are sequentially fired into the computational domain. The molecules enter the domain with probabilistically determined velocities. The velocity is composed of a constant freestream bulk velocity and a thermal velocity that is also probabilistically determined. The test particles do not undergo intermolecular collisions; however, TPMC is capable of simulating multiple deflections off the satellites surface. This is applicable to complex and concave satellite geometries as well as for areas where flow shadowing may occur on the satellite.

1.2.5.4. Surrogate Modeling The computational analysis methods used to simulate drag coefficients are computationally expensive. One simulation with a certain attitude and certain configuration can take a significant amount of time to complete. The user may need multiple instances of $C_{D}$ in order to propagate the orbit of their satellite too. The solution to this issue is to use a simulation technique along with an interpolation technique. By running multiple simulations it is possible to interpolate what the possible drag coefficients 
for all necessary configurations are. The Gaussian Process Regression (GPR) model is a great tool to accomplish this. GPR is a generic supervised learning method designed to solve regression and probabilistic classification problems [9].

Los Alamos National Laboratory (LANL) created the RSM toolkit that uses this technique in order to make the RSM a viable means of calculating drag coefficient [22]. The suite is capable of taking user inputs and implementing multiple GSI models. The LANL RSM toolkit uses MPI to parallelize simulations, making it very efficient and ideal for analysis with high performance computing clusters. With these abilities and resources, the code is capable of analyzing the most complex of satellite geometries in the FMF regime. The code can be found at the following GitHub link: https://github.com/ AndrewCWalker/rsm_tool_suite

\section{Los Alamos National LABORATORY RSM TOOLKIT}

The original Response Surface Model toolkit was developed at the Los Alamos National Laboratory to provide the community with a resource for simulating and modeling satellite drag coefficient in FMF. The code was used as the basis for the updated RSM software package created at West Virginia University (WVU). An important item to note is that this toolkit is dependent on the use of ASCII STL files. These files are a readable text file that contain all the information for the object being evaluated. 


\subsection{Stereolithography(STL) Files}

The Stereolithography (STL) file is the focal point of the simulation process for this software. Typically STL files come in two formats: ASCII and binary. For this software it is imperative that the ASCII format is used as binary is not supported. ASCII STL files use a very intuitive and very specific formatting. The premise behind the formatting of STL files is that an object can be made up of a series of triangular facets. These triangular facets make up a mesh of the object. In order to create the body and the mesh for the object to the users desired specifications, a Computer-Aided Design (CAD) software and/or a meshing software is needed.

Once the object has been created, the ASCII STL file takes on a specific format as shown in Figure 2.1. Each facet is defined by three vertices in the $\mathrm{x}$ $\mathrm{y}-\mathrm{z}$ Cartesian coordinate frame and a vector normal to the facet. The keywords "outer loop", "endloop", and "endfacet" define where the facet definition begins and ends. The keywords "solid" and "endsolid" define where the file begins and ends. Located next to "solid", is the name of the object. This name is irrelevant and can be changed without having any effect on the code. 


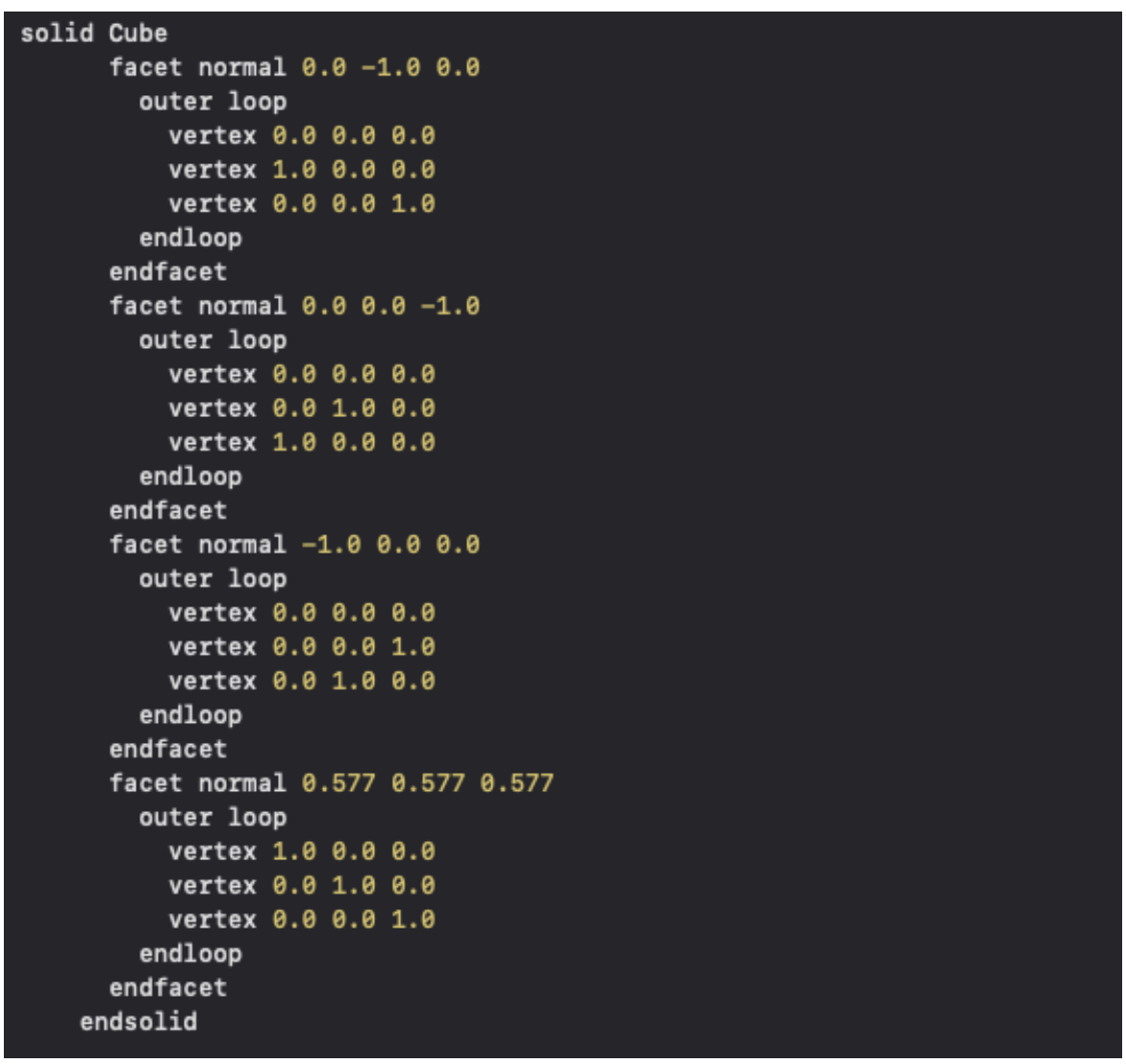

Figure 2.1: Example STL File, Slice of a Cube

\subsection{OVERVIEW}

The overview of the LANL RSM toolkit is illustrated in Figure 2.2. In order to operate the RSM, there are two user inputs that need to be configured before operation; an input script that defines the simulations and a user input for model evaluation. 


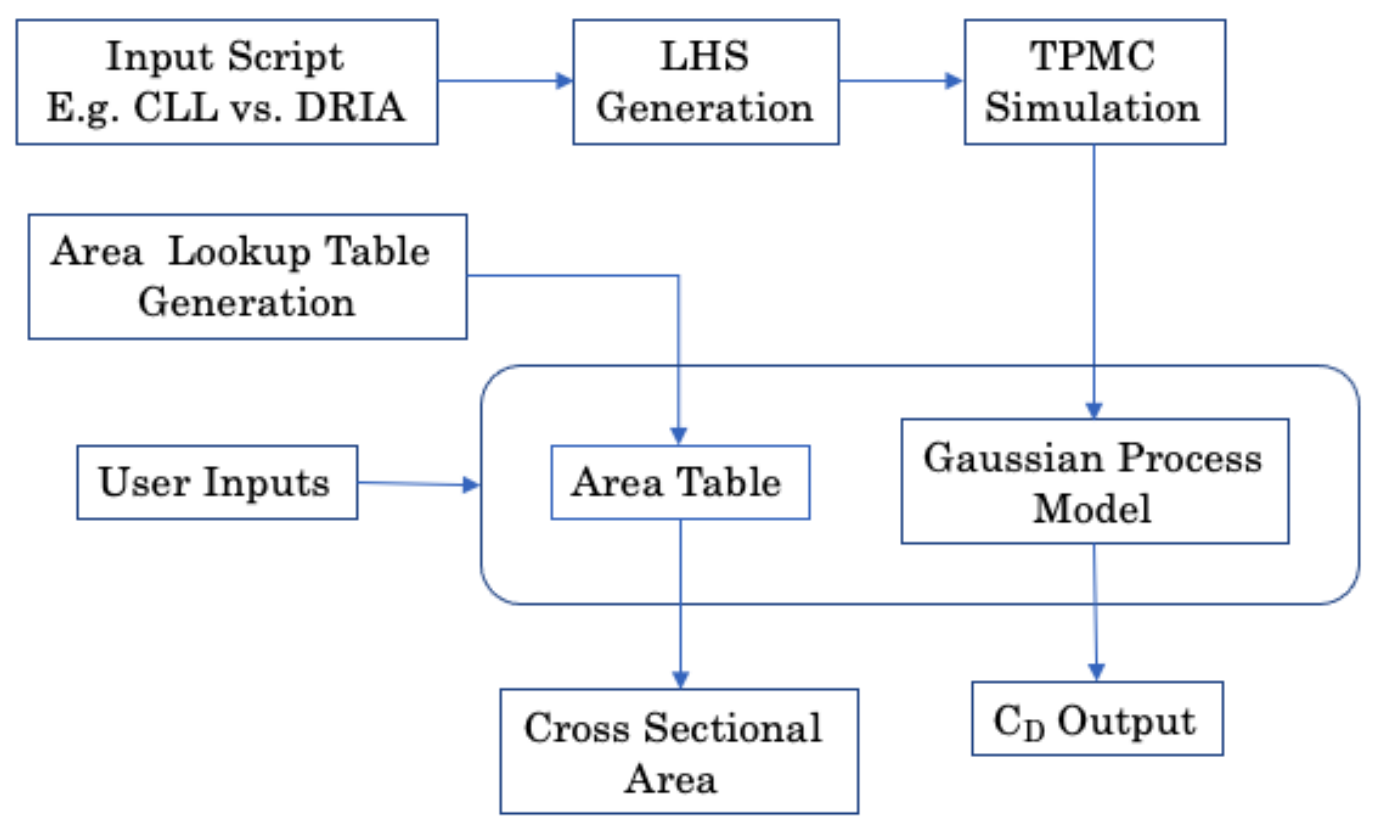

Figure 2.2: Flowchart of LANL RSM toolkit Operation

The LANL toolkit utilizes three essential codes that allow the user to perform simulations to create a regression model, calculate projected area of the object, and perform evaluation of the model with user inputs. The toolkit also contains several layers of directories. The top level directory, as shown in Figure 2.3, contains the three folders making up the three main codes utilized in the toolkit. For directory illustrations in this work, folders are filled blue and individual scripts are filled green. 


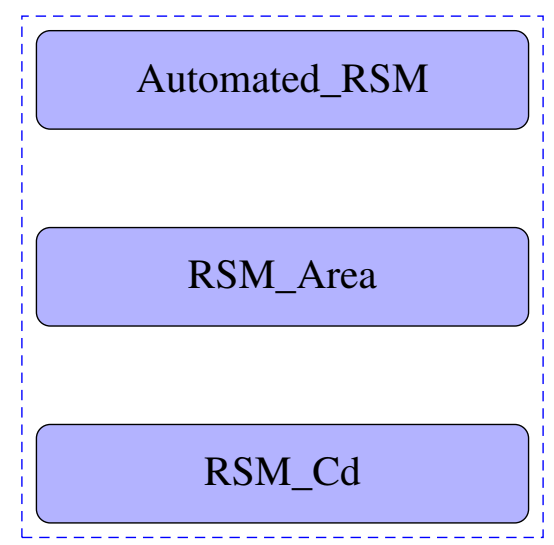

Figure 2.3: Top level Directory of LANL RSM Toolkit

\subsection{Automated RSM}

This folder includes a Python execution code that allows the user to perform simulations using TPMC. The TPMC code is written in C programming language. A regression model for multiple species of gas is created after the simulations are complete. The code in this section of the toolkit is capable of running the simulations in parallel with multiple processors via MPI. 


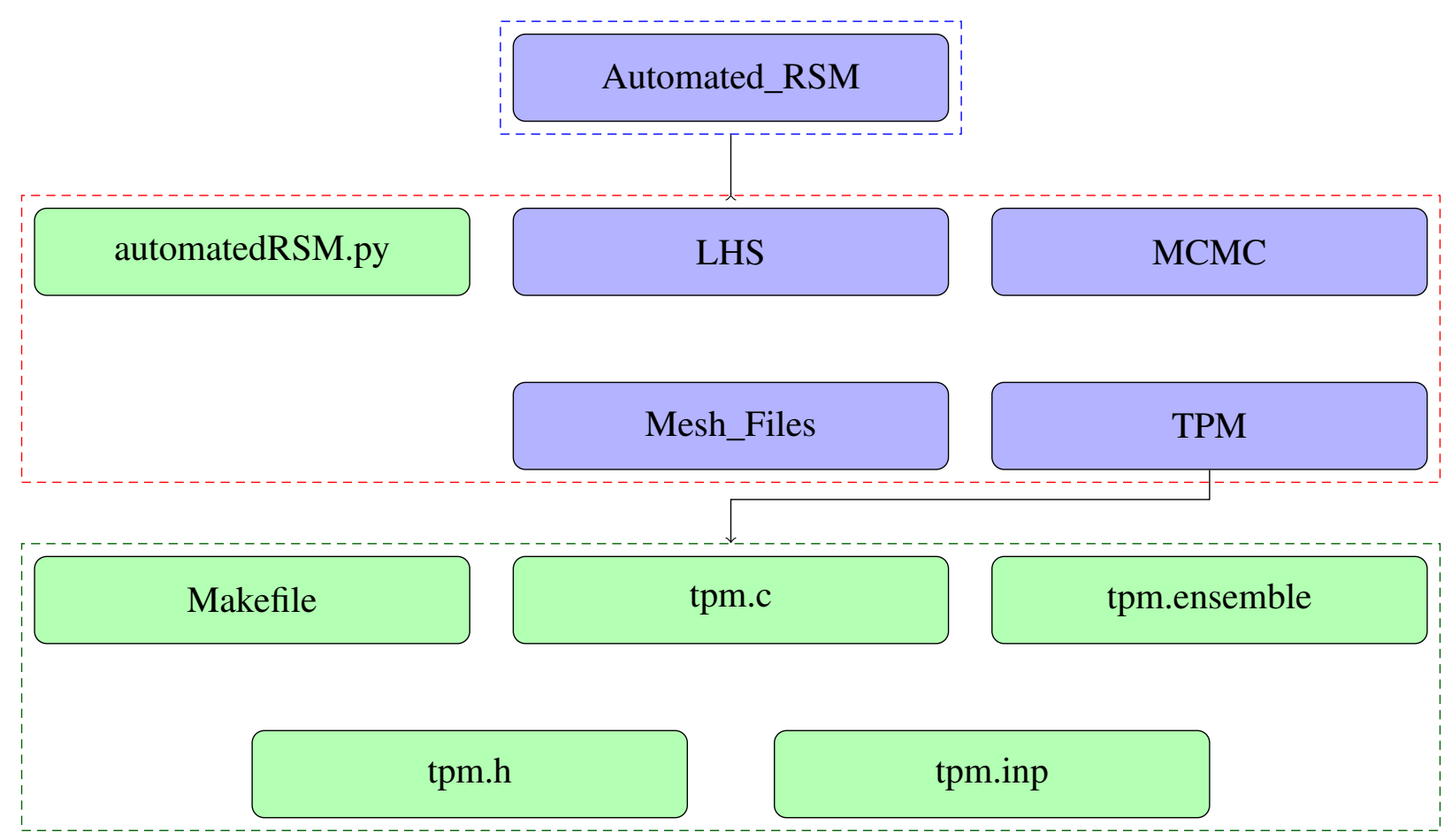

Figure 2.4: Automated_RSM folder of LANL RSM Toolkit

The automatedRSM.py is the script that executes the multiple codes in this folder. $L H S$ contains the Python scripts in order to execute Latin Hypercube Sampling (LHS) of the inputs for the TPMC simulations. MCMC contains the assorted Matlab® scripts to create the Gaussian Process Regression model. Mesh_Files is where STL files are placed. Finally, TPM contains the C code for the TPMC simulations and other related files including the tpm.inp file. This file is the input for the simulations and is read by the automatedRSM.py for the LHS.

The TPM folder includes multiple files the user must interact with. The tpm.c file is the code where the simulations of TPMC are done. This code is called twelve times from the Automated_RSM.py; six times, once for each species of gas, for the training data and then six times for the test data both of which are 
used to develop the GPR model.

The tpm.ensemble file is a text file also created by the Automated_RSM.py. Automated_RSM.py executes the LHS functions and then saves the values to this text file. Each line of tpm.ensemble is read by the tpm.c as the inputs for that particular simulation.

The Makefile and the tpm.h are both used to compile tpm.c. In order to perform compilation a user must have the appropriate $\mathrm{C}$ compilers installed on their computer or node and then execute $\$$ make. tpm.h contains the header information for the $\mathrm{C}$ code. In other words, this is where the functions are defined for the $\mathrm{C}$ code.

Lastly, tpm.inp is the text file that is used for inputs in both the Automated_RSM and tpm.c code. This file, as seen in Figure 2.5, contains the maximum and minimum values for the LHS, the filename/directory of the STL, and several other variables that are passed to tpm.c through this file. Automated_RSM needs to pass the species mole fraction, GSI model, and number of ensemble points for the TPMC simulation. As stated previously, tpm.c is executed six times for each species mole fraction at $100 \%$. Automated_RSM opens and rewrites this file to tell tpm.c the value of species mole fraction for the simulation being performed. 


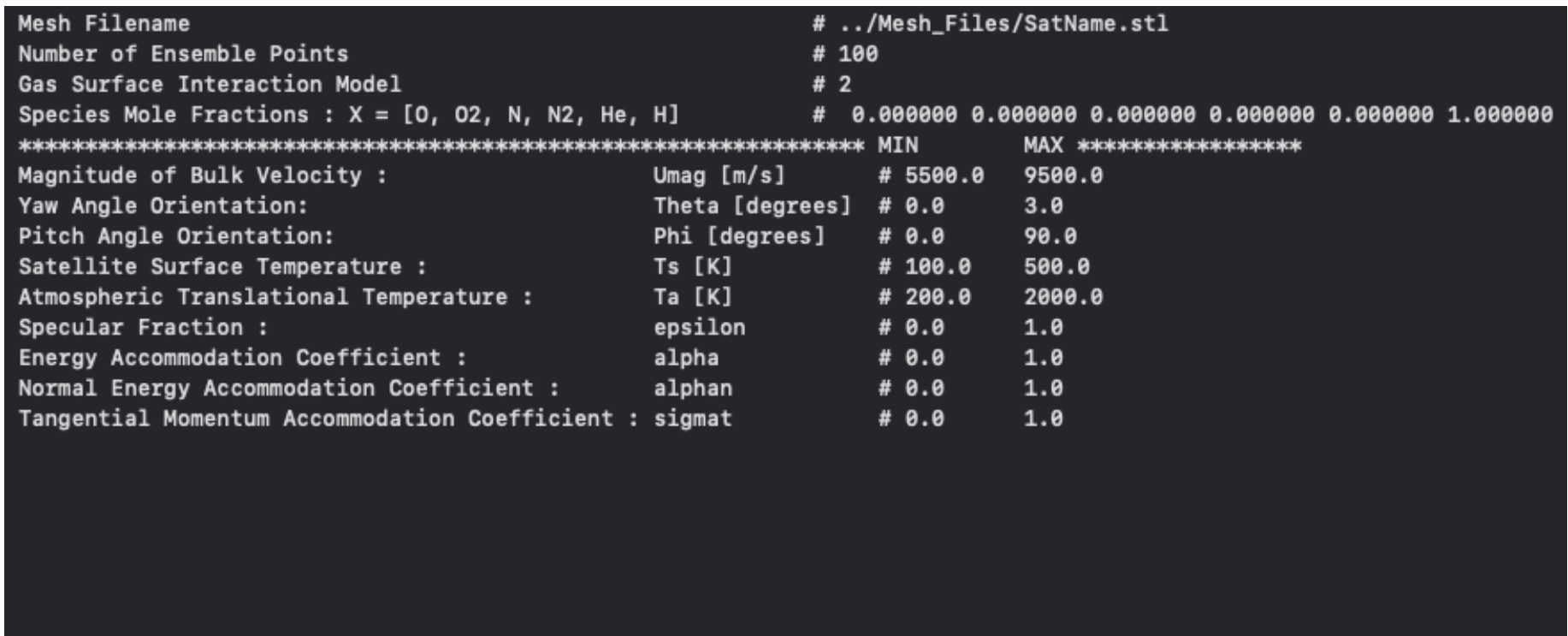

Figure 2.5: tpm.inp input file for tpm.c and Automated_RSM

Once the code finishes execution, all of the relevant model information is saved to a data file that will be used during the model evaluation.

\subsubsection{LANL REGRESSION MODEL}

As stated, within the Automated RSM the regression model is also created. The regression model uses a GPR model created in MATLAB® of which was developed in-house at LANL. The regression model models drag coefficients based on multiple inputs including velocity, temperature, mole fraction, etc.

The GPR was developed under several assumptions. First, the data is assumed to have extremely low variance. This allows the RSM to be able to nearly interpolate the training data. Secondly, it is assumed that the surface is continuous and fairly smooth. Finally, the GPR should quickly evaluate the fitted model at new input settings. LANL therefore developed their GPR based on one which was used to build approximations for complex computer simulators [32]. 
2.3.1.1. Formulation of the LANL REgression Model Formulation of the GPR assumes the results from the simulations have been normalized to have mean zero and variance one. A zero-mean Gaussian process model says that results, $\vec{y}$, have a multivariate Gaussian distribution with a particular structure for the covariance matrix that is a function of $X$ [22]. $X$ is some set of inputs given as an $m \times p$ matrix with rows given by $x_{i}$ of which have been normalized. The correlation given as a function of inputs is given by Equation 2.1

$$
R_{i, j}=\prod_{k=1}^{p} \theta_{k}^{4\left(x_{i, k}-x_{j, k}\right)^{2}}
$$

where $x_{i}$ and $x_{j}$ are the inputs and $\theta$ is the spatial correlation parameter. As the distance between these inputs goes to zero, $R_{i, j}$ goes to unity and the difference between values $y_{i}$ and $y_{j}$ at these inputs also goes to zero. Thus, this correlation structure ensures that a draw of $\vec{y}$ for the set of inputs will be a set of points on a continuous surface [22].

The simulations performed are a done with a Monte Carlo technique, therefore there is some noise around what is assumed to be a smooth underlying function. Thus, LANL slightly altered the covariance function to provide better results. The function used is shown in Equation 2.2.

$$
y \sim N\left\{\overrightarrow{0}, \sum(X)=\frac{1}{\lambda_{0}} R(X)+\frac{1}{\lambda_{\epsilon}} I\right\}
$$

where, $\lambda_{0}$ is the marginal precision, $\frac{1}{\lambda_{\epsilon}}$ is the variance, and $I$ is the identity matrix. Prediction can then be done based on the properties of a multivariate Gaussian distribution. The joint distribution of a set, where $\left(y_{i}, x_{i}\right)$ are the observed set and $\left(y_{i}^{*}, x_{i}^{*}\right)$ are the new points, with $x_{i}^{*}$ known and $y_{i}^{*}$ unknown, is 
given by Equation 2.3 .

$$
\left(\begin{array}{c}
\vec{y}^{*} \\
\vec{y}
\end{array}\right) \sim N\left\{\overrightarrow{0},\left[\begin{array}{cc}
\sum\left(X^{*}\right) & \sum\left(X^{*}, X\right) \\
\sum\left(X^{*}, X\right)^{\prime} & \sum(X)
\end{array}\right]\right\}
$$

The prediction of $\vec{y}^{*}$ is then determined given the conditional distribution of $\vec{y}^{*} \mid \vec{y}$ which is Gaussian, $\vec{y}^{*} \mid \vec{y} \sim N\{\mu, \Psi\}$. Where $\mu$ and $\Psi$ are the following:

$$
\begin{gathered}
\mu=\sum\left(X^{*}, X\right) \sum(X)^{-1} \vec{y} \\
\Psi=\sum\left(X^{*}\right)-\sum\left(X^{*}, X\right) \sum(X)^{-1} \sum\left(X^{*}, X\right)^{\prime}
\end{gathered}
$$

\subsection{Projected Area}

The LANL RSM toolkit uses geometry of the object to calculate the projected area of a given yaw and pitch, written in $\mathrm{C}$ programming language. A range of yaw and pitch is given with a certain resolution of each that ultimately creates a lookup table of areas. This table can be bi-linearly interpolated to obtain a projected area that is needed to find the ballistic coefficient of the object. 


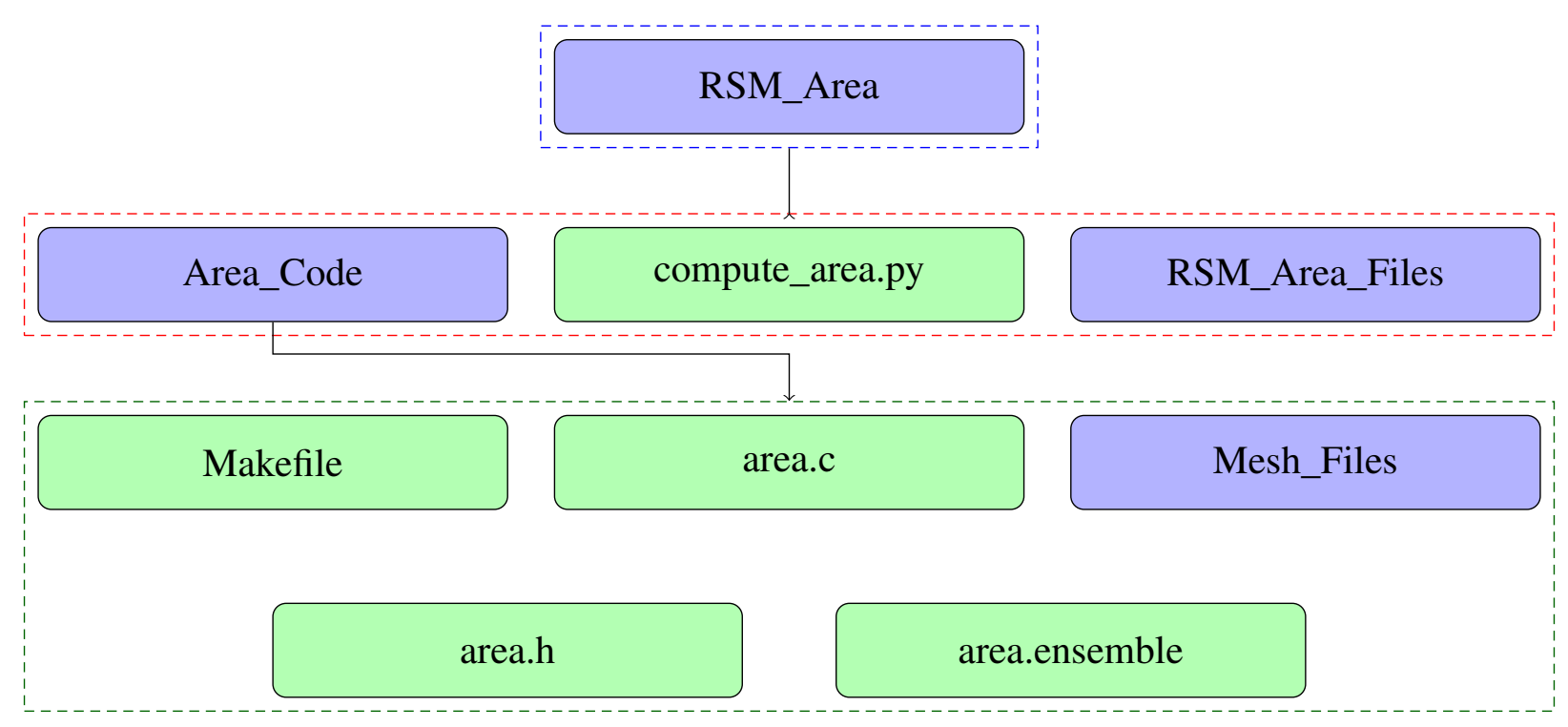

Figure 2.6: RSM_Area folder of LANL RSM Toolkit

The executable script for this part of the toolkit is compute_area.py. It passes hardcoded inputs to the area.c code, located in the Area_Code folder, that are declared directly within the compute_area.py script. RSM_Area_Files is an empty folder where the user can place data files generated by the code.

Area_Code is the folder that contains the source code to calculate the projected areas for the lookup table. The STL file used in the TPMC simulations must be placed in the Mesh_Files folder. Then, with the appropriate $\mathrm{C}$ compilers installed, area.c needs to be compiled by executing $\$$ make. The area.c code also associates with the header file area.h to declare the functions within the $\mathrm{C}$ code. The area.ensemble is a text file where the area information form area.c is saved. Once the code is finished running a data file is output containing the lookup table for mode evaluation. 


\subsection{Model Evaluation}

The final section of code is used to evaluate the model created from the Automated RSM. This code was written in the $\mathrm{C}$ programming language as well. The user can input the desired precise values for evaluation into the $R S M$.inp file. Execution returns a single, accurate, drag coefficient as well as ballistic coefficient. This code evaluates the multi-variate input with a Langmuir or Freundlich adsorption model taking into account fractional surface coverage of adsorbed oxygen.

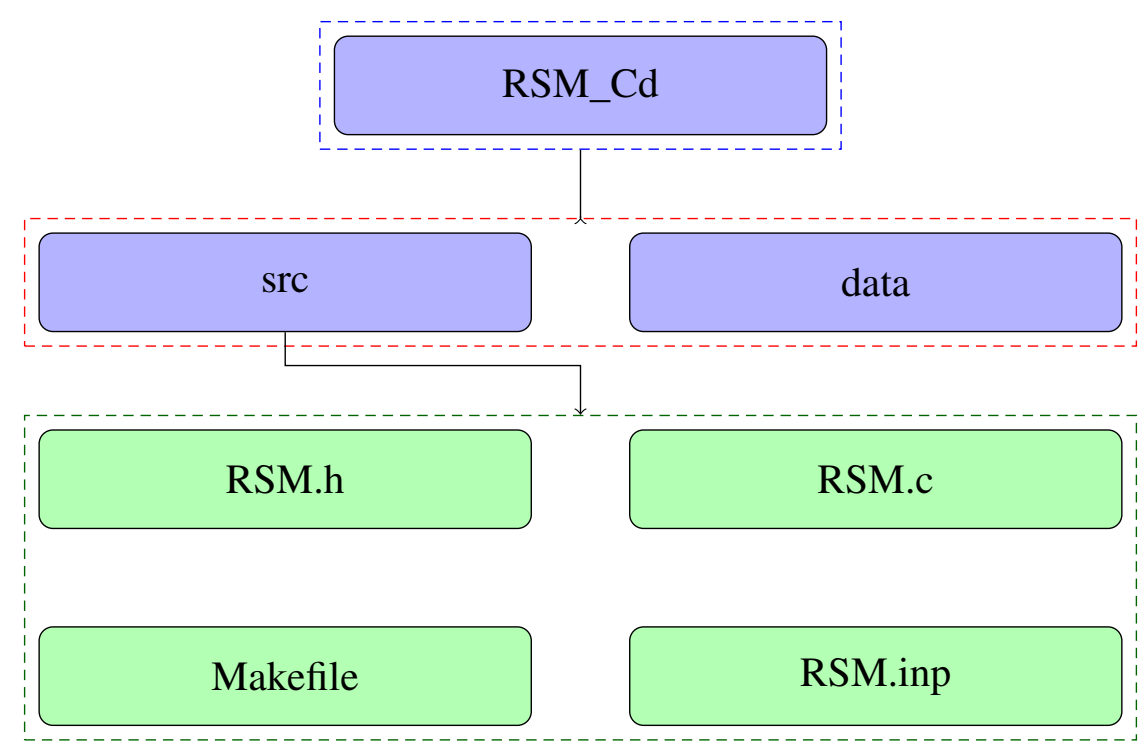

Figure 2.7: RSM_Cd folder of LANL RSM Toolkit

data is the folder where the user must place the Automated_RSM and RSM_Area data files to be read by the RSM.c code. $\operatorname{src}$ contains the relevant source code for analyzing the MATLAB® GPR model and calculating the drag coefficient. This code must also be compiled with the $\$$ make command. Figure 2.8 is a example of the RSM.inp file. This input file is line dependent and must keep a strict formatting. 


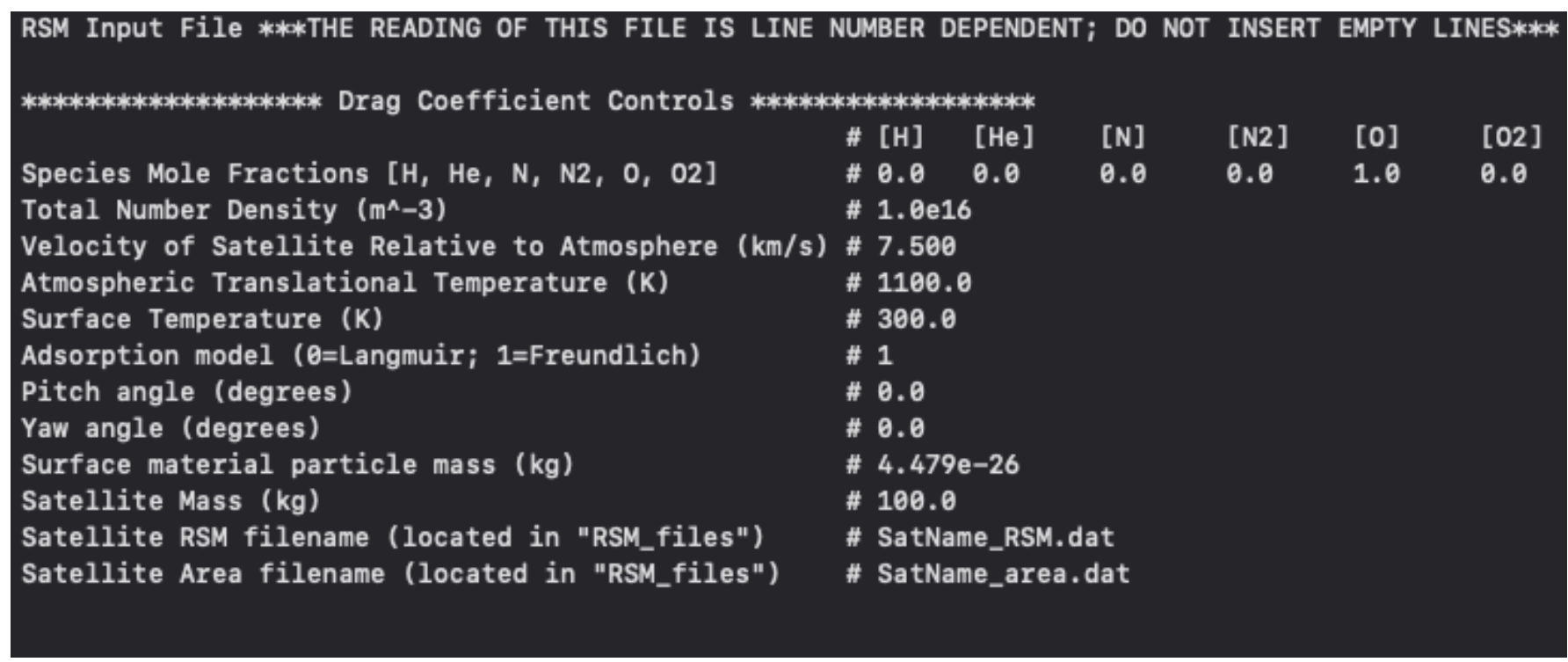

Figure 2.8: RSM.inp input file for RSM.c

\subsection{LIMITATIONS OF LANL RSM TOOLKIT}

There are certain limitations to the LANL RSM toolkit. For instance, one issue that arose during testing of the code was that the RSM was giving false information, or in some cases crashing. TPMC simulates particles colliding with the surface of an object. Gaps or holes in the object can cause particles to become trapped and give false readings as they bounce inside the object. When this occurs it is said the mesh is non-watertight. This issue does not reside withing the tpm.c code, but arises from the STL file generation. In order to make sure the user does not run into this issue, a function that checks the water-tightness of the mesh can be developed.

While it is possible to model satellites with rotating components, it can not be done automatically within the LANL RSM. The user must create a new satellite for each individual simulation. To help model this better, a rotation algorithm can be implemented that will allow the user to predict the drag at all desired 
configurations of the satellite.

The LANL RSM toolkit also has the limitation of using MATLAB® code. This code can be computationally and monetarily expensive. In this work, the code has been made to an open-source software that hopefully will allow the RSM to become a community standard that all agencies across the globe can use to more accurately model the drag coefficient of their satellites.

The toolkit was also developed for LANL's personal needs and does not give much consideration to the end user. For instance, the suite has multiple places to compile code and execute. Ideally, the user should only have to install the suite one time with everything compiled and ready for execution. There should also only be execution of a single code for simulation and model creation. Then once the model is created there should be another code for model evaluation along with projected area calculation. A lot of the inputs and definitions are hardcoded into the multiple scripts as well. This makes the user have to parse through the code to find definitions and variable declarations. Documentation is also lacking from the toolkit. While, there are "README" files included with the suite, they do not divulge detailed operation of all the codes. Implementation of these features would turn the suite into software package that a new user could pick up and start using with relative ease.

\section{WEST VIRGINIA UNIVERSITY'S RSM}

\section{SOFTWARE PACKAGE}

Once operational, the LANL RSM suite was developed into a software package with extended capabilities into complex satellite geometries with rotating com- 
ponents. The software has also been developed around providing a new user with an easily navigable environment. Furthermore, the Gaussian Process Regression model is now created via Python's Scikit-learn module. This is beneficial as it is less computationally expensive, more efficient, and open source. This new suite allows the user to not only execute and automatically develop RSM models, but also to execute individual scripts independently such as: projected area calculations, STL rotation and generation, model creation, and model evaluation. The new flowchart for operation of the WVU RSM toolkit is shown in Figure 3.1. Comparing this flow chart to the one in Figure 2.2, there are a couple notable differences in operation. Rotation has been added to the toolkit and the area script from the LANL RSM has been removed.

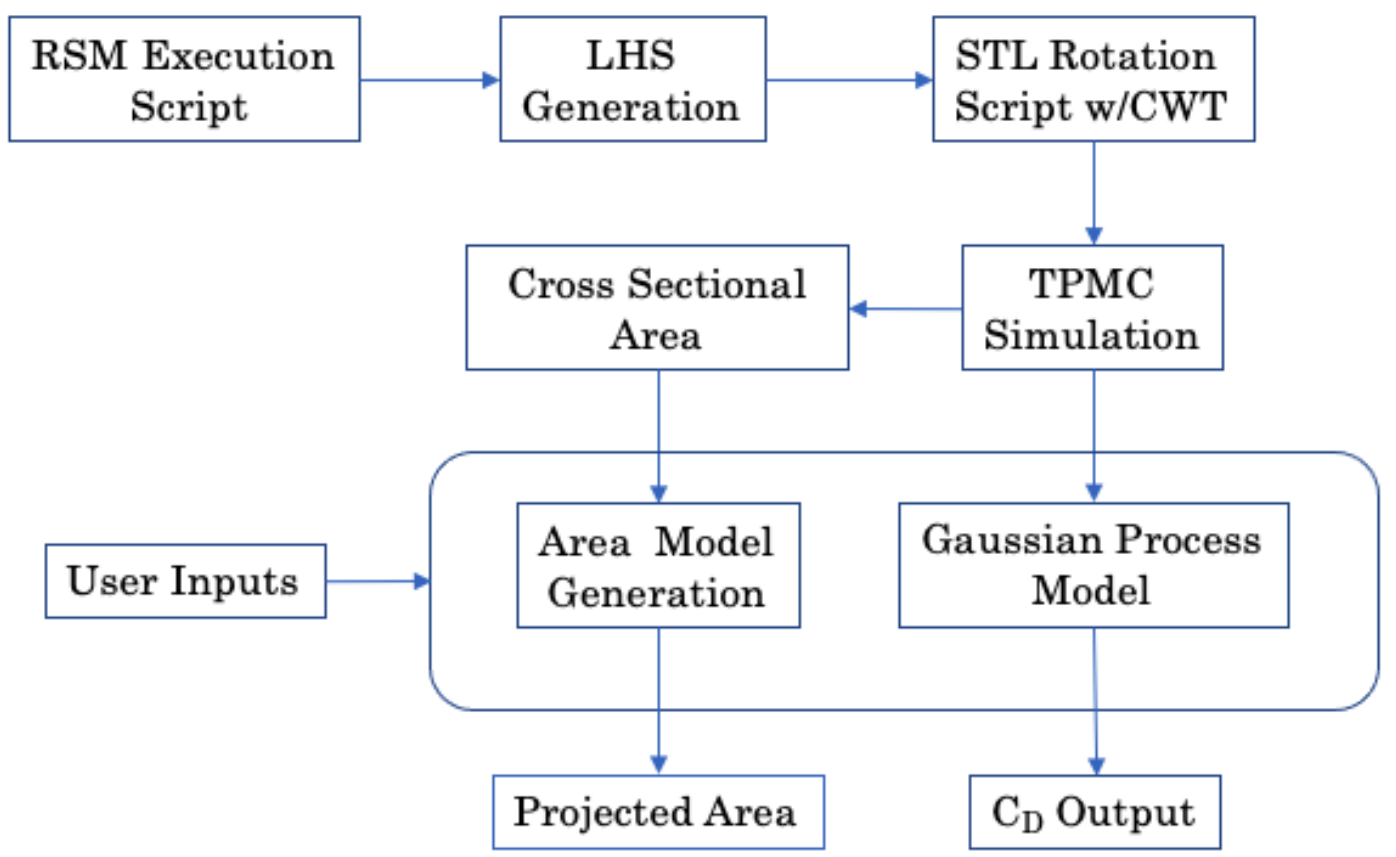

Figure 3.1: Flowchart of WVU RSM Toolkit Operation 


\subsection{EXTENSION TO COMPLEX GeOMETRIES}

The largest addition to the RSM suite is the ability to evaluate complex geometries. Complex geometries refer to objects with rotating components such as, but not limited to, solar panels. Allowing for rotating components to be a part of the regression model creates an n-dimensional number of inputs. This proves to be a challenge with a lot of the original LANL code. To overcome this, new file types were introduced to the suite such as JSON and HDF5 files. The readability of these, along with metadata attributes, make them ideal for information exchange within the software package.

The implementation of component rotation requires an understanding of how ASCII STL files are structured. The user must also have a strong understanding of the size and structure of their object as well as spatial awareness of each component. This is because components of the object are rotated individually and then combined to make a whole structure.

The code implements a rotation algorithm and is performed over the number of samples created during the LHS generation. The algorithm was further extended to combine all of the components to form the whole structure of the object as well. This process creates a new STL that is used for a single simulation. If there are multiple simulations being performed, that many STL files will be created. For instance, an n-number of ensemble points will generate an n-number of STL files for simulation in the RSM code.

\subsection{ROTATION OF STLS}

Often, satellites are not fixed bodies. They can have components that rotate such as solar panels, dishes, or antennas. The ability to rotate STL files allows 
for the creation of a regression model that can give a user the ability to look up drag coefficients without the need to recreate an object every time they need to simulate a different configuration. A rotation algorithm was created for this software that can rotate components of a satellite and output new STL files for either TPMC simulation or for the users own personal use.

In order to rotate an STL file, an algorithm that was created by James A. Tancred at the Air Force Research Laboratory is used [36]. Originally the code was developed in MATLAB ${ }^{\circledR}$ and the algorithm cleverly uses several text files as inputs. The input files are comprised of a "parent" and "child" file which call and assign names of the components being rotated, respectively. A "deflection" text file is also used to define the deflections for each component. The user may input multiple instances in this file to create multiple STL files as well. Two "hinge point" files are used to define the line about which components are rotated. The first file defines the point at which a line begins and the second defines the point at which it ends. These two points create a vector that the rotation occurs about. The positive direction of rotation then follows the righthand-rule. The code also has the ability to display the new and old STL files showing where the rotation occurs.

To actually perform the rotation, each facet needs to be rotated using direction cosine matrices. First, the hinge line vector needs to be defined with respect the "world origin", a point for reference in the code. The user has the option to define different world origins; however, the natural choice is to use $(0,0,0)$. From there, three direction cosines can be calculated by the angles given in Equation 3.1. These angles are measured by the vector, $\vec{h}$, between the hinge line and the world origin 


$$
\begin{aligned}
& \alpha=\frac{180}{\pi} \cos ^{-1} \frac{h_{x}}{|\vec{h}|} \\
& \beta=\frac{180}{\pi} \cos ^{-1} \frac{h_{y}}{|\vec{h}|} \\
& \gamma=\frac{180}{\pi} \cos ^{-1} \frac{h_{z}}{|\vec{h}|}
\end{aligned}
$$

The rotation angles may now be calculated with the following equations:

$$
\begin{gathered}
\theta_{1}=\frac{180}{\pi} \cos ^{-1}\left(\frac{\cos ^{2}(\alpha)+\sin ^{2}(\gamma)-\cos ^{2}(\beta)}{2 \cos (\alpha) \sin (\gamma)}\right) \\
\theta_{2}=90-\gamma \\
\theta_{H}=\theta_{\text {deflection }}
\end{gathered}
$$

These three angles can then be used to create the appropriate Eulerian rotation matrices.

$$
\begin{aligned}
& R_{1}=\left[\begin{array}{ccc}
\cos \left(\theta_{1}\right) & \sin \left(\theta_{1}\right) & 0 \\
-\sin \left(\theta_{1}\right) & \cos \left(\theta_{1}\right) & 0 \\
0 & 0 & 1
\end{array}\right] \\
& R_{2}=\left[\begin{array}{ccc}
\cos \left(\theta_{2}\right) & 0 & \sin \left(\theta_{2}\right) \\
0 & 1 & 0 \\
-\sin \left(\theta_{2}\right) & 0 & \cos \left(\theta_{2}\right)
\end{array}\right] \\
& R_{3}=\left[\begin{array}{ccc}
1 & 0 & \\
0 & \cos \left(\theta_{H}\right) & \sin \left(\theta_{H}\right) \\
0 & -\sin \left(\theta_{H}\right) & \cos \left(\theta_{H}\right)
\end{array}\right]
\end{aligned}
$$

The vertices of each facet can now be rotated with Equation 3.8 . 


$$
\mathbf{X}=R_{1}^{-1} R_{2}^{-1} R_{3} R_{2} R_{1}\left(\mathbf{X}_{\mathbf{b}}-\mathbf{\Delta}\right)+\Delta
$$

$\mathrm{X}$ is the new set of vertices for the facet, $R_{n}$ is the matrix representing the elemental rotations about the respective axes $\mathrm{x}, \mathrm{y}$, or $\mathrm{z}, \mathbf{X}_{\mathbf{b}}$ is an array of the original vertex points, and $\Delta$ is the distance between the second hinge point and the world origin. Once the vertices have been rotated, the normal vectors of the facets also need to be rotated. To do this, the same approach and equations are used as the vertices.

After this is accomplished the new STL component can be printed in the same aforementioned formatting. Multiple components can also be combined to create larger structures that can be used for simulations. To make sure there are no errors with this method, a "Check Water-Tightness" function was created and is performed on each STL file.

\subsection{Check Water-Tightness}

Another addition to the package is the ability to check water-tightness of the object. Also sometimes referred to as manifold surfaces, the surfaces of the object can not have any gaps or holes in them. Breaks in the structure can cause particles to become trapped and bounce around inside the object giving false data. To overcome this, a basic function was created to make sure each side of a triangular facet is shared by exactly two triangles. If a mesh is not water-tight, then the program will stop execution with an error message. 


\subsection{WVU REGRESSION MODEL}

The regression model creation was done using a Gaussian Process Regression model via Python's Sci-Kit Learn module. An important benefit of doing the GPR modeling in Python is that it provides uncertainty bounds as well. These bounds are a good indicator of the performance of the GPR. For the GPR to function, the prior covariance function needs to be specified. This is done so by the use of a predefined kernel. Kernels determine the shape of the prior and posterior of the GPR. According to the Sci-Kit Learn documentation, "they encode the assumptions on the function being learned by defining the 'similarity' of two data points combined with the assumption that similar data points should have similar target values" [9]. Python's Sci-Kit Learn module contains all the necessary functions to accomplish the creation of the GPR. Through much trial and error, the WVU ASSIST lab has determined that the most appropriate kernel for modeling drag coefficients in FMF is the Matern kernel. The Matern kernel is a stationary kernel and a generalization of the Radial Based Function (RBF) kernel [9]. The kernel is given by the following function, defined in Equation 3.9 .

$$
k\left(x_{i}, x_{j}\right)=\frac{1}{\Gamma(\nu) 2^{\nu-1}}\left(\frac{\sqrt{2 \nu}}{l} d\left(x_{i}, x_{j}\right)\right)^{\nu} K_{\nu}\left(\frac{\sqrt{2 \nu}}{l} d\left(x_{i}, x_{j}\right)\right)
$$

where $l$ is a length scale parameter greater than zero, $d\left(x_{i}, x_{j}\right)$ is the Euclidean distance, $K_{\nu}()$ is a modified Bessel function, and $\Gamma(\nu)$ is the gamma function. The parameter $\nu$ controls the smoothness of the function. In this work $\nu$ is set to 2.5, a value that is common for twice differentiable functions. As $\nu$ approaches infinity, the Matern kernel converges to the RBF kernel. When $\nu=\frac{1}{2}$, the 
Matern kernel becomes identical to the absolute exponential kernel [9].

\subsubsection{UNCERTAINTY}

Scikit-learn library has a functionality named "GaussianProcessRegressor," which can do Gaussian Process Regression and provide an estimate of both the mean and the uncertainty, also known as standard deviation, of the prediction. If too few ensemble points are used in the training process, i.e., if the ensemble points are sparsely distributed, then the size of the predicted uncertainty is large. As such, the configuration and the number of ensemble points need to be carefully designed.

The prediction of the posterior is found with Bayes' theorem by conditioning on the prior and the observations. The predictive posterior distribution corresponds to the test inputs, $\mathbf{X}_{*}$, for the observation dataset $\left\{\mathbf{X}_{o}, \mathbf{F}_{o}\right\}$, and is given by Equation 3.10 [31]

$$
\begin{gathered}
p\left(\mathbf{F}_{*} \mid \mathbf{X}_{*}, \mathbf{X}_{o}, \mathbf{F}_{o}\right)=\mathcal{N}\left(\mathbf{F}_{*} \mid \mu_{*}, \boldsymbol{\Sigma}_{*}\right) \\
\mu_{*}=\mathbf{K}_{*}^{T}\left(\mathbf{K}_{o}+n_{l}^{2} \mathbf{I}\right)^{-1} \mathbf{F}_{o} \\
\boldsymbol{\Sigma}_{*}=\mathbf{K}_{* *}-\mathbf{K}_{*}^{T}\left(\mathbf{K}_{o}+n_{l}^{2} \mathbf{I}\right)^{-1} \mathbf{K}_{*}
\end{gathered}
$$

where $\mathbf{K}_{*}=\mathbf{K}\left(\mathbf{X}_{o}, \mathbf{X}_{*}\right), \mathbf{K}_{o}=\mathbf{K}\left(\mathbf{X}_{o}, \mathbf{X}_{o}\right)$, and $\mathbf{K}_{* *}=\mathbf{K}\left(\mathbf{X}_{*}, \mathbf{X}_{*}\right)$. Hyperparameters $l$ and $n_{l}$ are found by maximizing the log marginal likelihood of the training data. The value $\mathbf{I}$ is representative of the identity matrix and the value of $\Sigma_{*}$ is the measure of the uncertainty of the model. 


\subsection{Model Evaluation}

The model evaluation code uses an emulator function that reads the files generated from the regression model creation code. These files provide the adsorbate $C_{D}$ and the clean surface $C_{D}$. Using the aforementioned total drag coefficient approach, the code provides the user with the final drag coefficient. The same evaluation code also outputs the projected area and the uncertainty of the model.

\subsection{Compilation/Installation of Code}

A significant difference between the LANL RSM suite and this software package is the build of the package. To elaborate, the suite is now a software package in the sense that compilation and installation of the package is backed by a series of scripts that evaluate the users environment to make sure execution is successful. This is still a high level package that is not recommended for desktop environments. Instead, it is recommended to be used with high performance clusters utilizing multiple nodes and processors; however, with the correct modules the user is very much able to run this software on a desktop environment. The package is also capable of giving the developers debugging information in case any issues arise.

The software package can be obtained by a GitHub repository link given in Appendix B. Once downloaded, the package needs to be set up. This is a one time procedure the user must preform to compile the appropriate TPMC code. It should be noted that whichever modules the user uses to compile the code must also be used to execute the code as well. 


\subsubsection{MOdules REQUiRED}

In order to compile, the following module types need to be accessible and loaded on to a desktop, cluster, and/or node. Also, the Python modules need to be backed with numpy, pandas, h5py, scipy, and sklearn. All other modules used are a part of the Python standard libraries. Another requirement is that GSL, used for random number generation, and MPI, used for parallelism, be included with the $\mathrm{C}$ compilers. If any of these are not present, they can be downloaded from the internet or uploaded by a cluster specialist. The main compilers needed are:

1. C code compiler, with MPI implementation

2. Python 3.x interperter

3. HDF5 library for $\mathrm{C}$

Examples of the appropriate packages can be found under the load_sources.sh script found in the $R S M_{-} T P M C$ folder. This script can also be modified and used for the user's own purposes. Currently, as downloaded, the script can be called with the following command and output the following modules:

\$ source load_sources.sh intel19

\$ module list

lang/gcc/9.3.0 lang/python/intelpython3_2019.5 I

lang/intel/2019 libs/hdf5/1.12.0_intel19

These are the modules and directories used on the WVU cluster. The user should load in modules with respect to their cluster or machine. 


\subsubsection{COMPILATION}

There is only one code that needs to be compiled before use and that is the TPMC code. The filename for this code is called tpm.c and can be found under the RSM_TPMC/tpm/src working directory. In order to compile, the user needs to follow the following instructions. Any errors that may arise during compilation are noted by the autotools build system.

1. Navigate so the current working directory is ./RSM_TPMC/tpm/.

2. Create a folder for compilation, this is not mandatory, but it is a good practice to separate the compiled code from the sources.

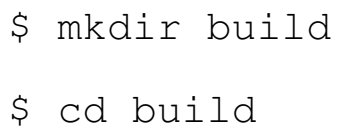

3. Execute the configure with appropriate flags. mpiicc is the compiler wrapper over Intel compilers and mpicc is the Intel MPI wrapper over GCC compilers. The code needs HDF5 and GSL as well, for example, using Intel compilers:

$\$ C C=m p i i c c . . /$ configure

The configure will figure out the right values for HDF5 and GSL libraries, otherwise you can also explicitly insert them like this:

\$ CC=mpiicC LIBS="-lhdf5 -lm-lgsl -lgslcblas"। ../configure 
4. Execute make to build the code

\$ make

5. After compilation the executable is located at: build/src with the name "tpm"

You can use this binary directly or you can install it. For installing the code you can decide a good place for installation with:

\$ CC=mpiicC LIBS="-lhdf5 -lm -lgsl -lgslcblas" ।

../configure --prefix=\$HOME/.local

and after make, install the code with:

\$ make install

Otherwise, this executable code can be used to run the package. For the rest of this work the binary file is used directly and can be found in the directory: RSM_TPMC/tpm/build/src/tpm.

All of the Python scripts should be executable upon downloading from the repository. If for some reason the scripts are not, $\$$ chmod $+x$ needs to be performed on the following scripts to make them executable. If all are executable, the user is ready to begin utilizing the software.

- model_evaluation_script.py 
- projected_area.py

- regression_model.py

- rotate_stl.py

- rsm_run_script.py

\subsection{NAVIGATION}

The software contains multiple directories that user must know in order to traverse the RSM. The diagram in Figure 3.2 illustrates the top level directory of the software package.

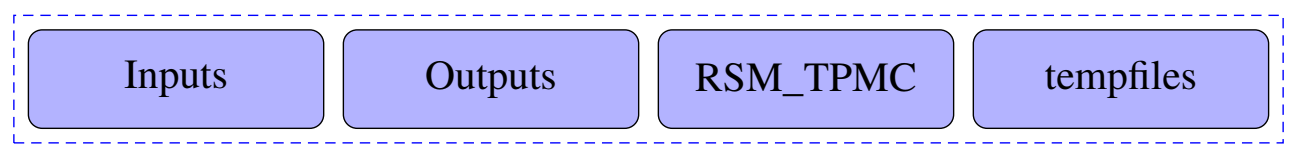

Figure 3.2: Top Level Directory of WVU RSM Toolkit

\subsubsection{OVERVIEW}

In the top level directory there are four folders. Inputs, Outputs, RSM_TPMC, and tempfiles. The Inputs folder is where the user will change values as they see fit and is the only place where the user has to change anything in the software. The Outputs folder is where everything of importance is output by the various codes. RSM_TPMC is where all the code is contained. Finally, tempfiles is a folder where several temporary files are kept. The user never needs to change anything within here. For informational purposes though, the items contained within the tempfiles folder are variables that are passed from Python scripts to the tpm code, the STL files created by the rotation Python script, and the ensemble information created by the LHS. 


\subsubsection{INPUTS}

How to use the Inputs folder is shown under the example execution section in the results of this work. This is an illustration and explanation of the structure of the Inputs folder. The Inputs folder contains the items shown in Figure 3.3 .

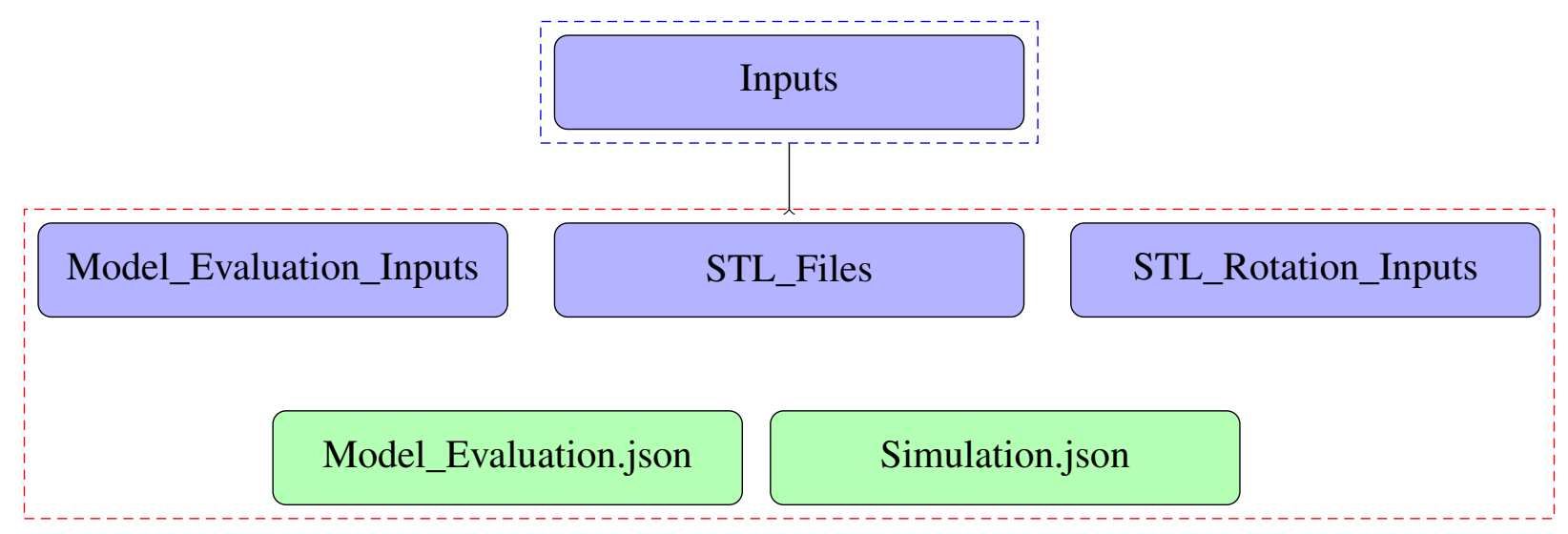

Figure 3.3: Inputs Directory of WVU RSM Toolkit

The Model_Evaluation_Inputs folder contains two items necessary to produce the drag coefficient results. The first item is the Model_Input_Data.csv file, which is used to calculate the drag coefficient. The second is the Regression_Models folder. This folder is where the user places the regression models generated from the simulation scripts.

The STL_Files folder is where all STL file types go. Whether individual components or a single structure object, this is where the user will place their STLs. It is worth reiterating that this is not where the rotation script outputs STLs. Since that script outputs one STL per simulation, they are stored and deleted in the tempfiles folder.

STL_Rotation_Inputs consists of three files that are necessary to define how rotations are performed. 
The Model_Evaluation.json and Simulation.json files define the different values and ranges used to create and evaluate the model. Their structure is explained later in this work.

\subsubsection{Outputs}

The Outputs folder is where all pertinent information from the scripts is output.

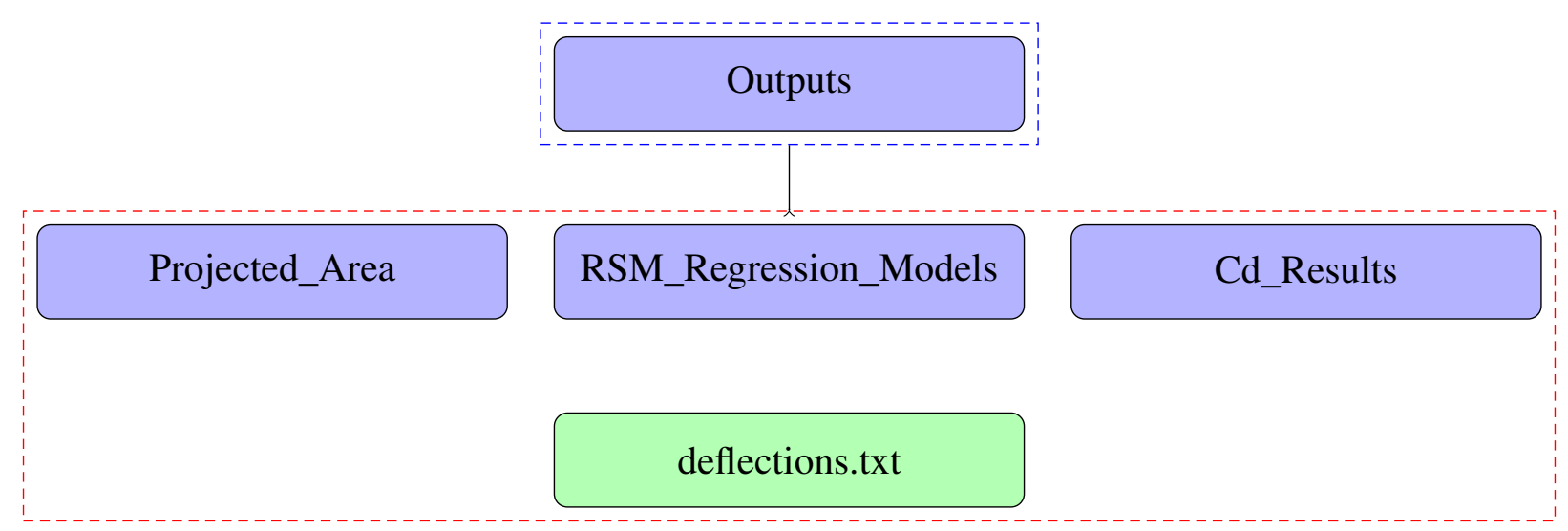

Figure 3.4: Outputs Directory of WVU RSM Toolkit

Projected_Area contains the file produced by the tpm code with the projected area of each simulation. This file is called Aout.dat. The user does not need to do anything with this file, but the projected_area.py code does use it to create a model for projected area calculation. The final projected area results from model_evaluation_script.py are also output to this folder as a text file.

RSM_Regression_Models is where the regression models from regression_models.py are output. After each run, thirty files defining the model are added to this folder.

Cd_Results is where the drag coefficients from model_evaluation_script.py are output as a text file. 
The deflection.txt file is created from the Latin Hypercube Sampling. During the LHS sampling, the deflections of each component for each ensemble point is created and saved to this text file. This is for reference purposes only. The actual deflection.txt is not used by any other scripts.

\subsubsection{RSM_TPMC}

RSM_TPMC is the section of the software package that contains all of the code. There are many parts of $R S M \_T P M C$ that are not useful to the user and only the relevant directories are discussed in this section. A diagram of the folder is shown below in Figure 3.5. There are multiple sub-directories that contain the code being used.

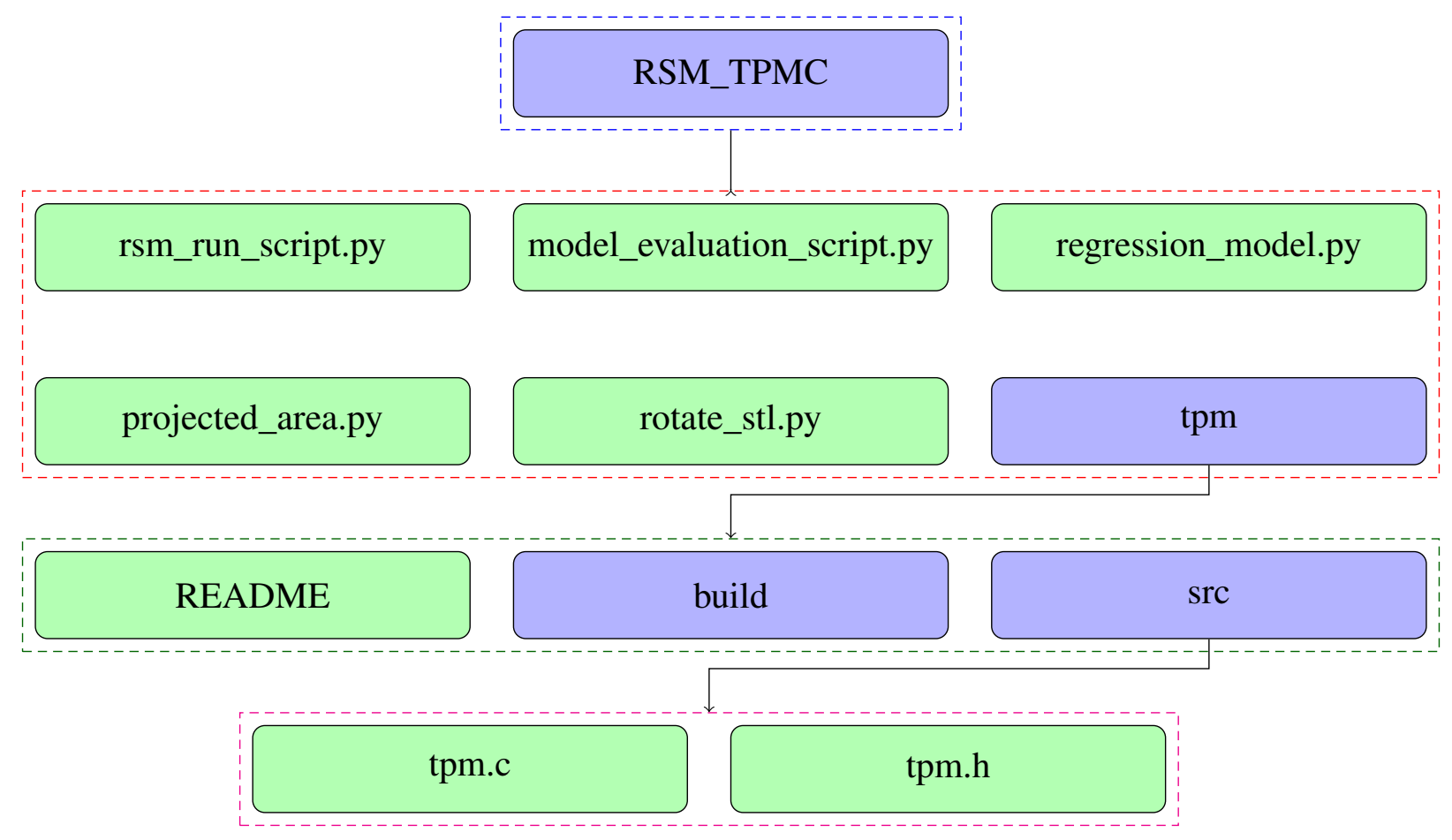

Figure 3.5: RSM_TPMC Directory of WVU RSM Toolkit

The main scripts that the user will call are the rsm_run_script.py and the 
model_evaluation_script.py. Both of these are executables that the user will have to execute in order to create the model and then evaluate it. The regression_model.py and rotate_stl.py run automatically from the rsm_run_script.py. These can also be ran independently if the user wishes to just rotate an STL or create a regression model.

The tpm folder is the folder that contains the TPMC code. In this folder, the three important items are the README, build, and src. The README file has the aforementioned steps to compile the code. build is the folder created by the user during installation where the executable TPMC code is kept. src contains the tpm.c code and its header file, tpm.h. This is the simulation code that is called by the rsm_run_script.py

\subsection{Simulation ExeCUTiON}

The setup, execution, and evaluation of the code to return drag coefficients and projected area is a multi-step process. Several inputs are required and the process changes slightly with rotation versus no rotation. The execution of the software also requires specific commands to ensure there are no directory conflicts within the various codes.

\subsubsection{Simulation InPUTS}

The software requires multiple user defined inputs to operate, all found in the Inputs folder. In order to define the inputs used for the simulations, the user needs to change the Simulaion.json file. This file is a JSON file to provide the user with flexibility in inputting the relevant information. For instance, line order and values don't matter. The only thing that matters is the format of the file 
and that the option/variable names be kept the same. Figure 3.6 is an example of this file.

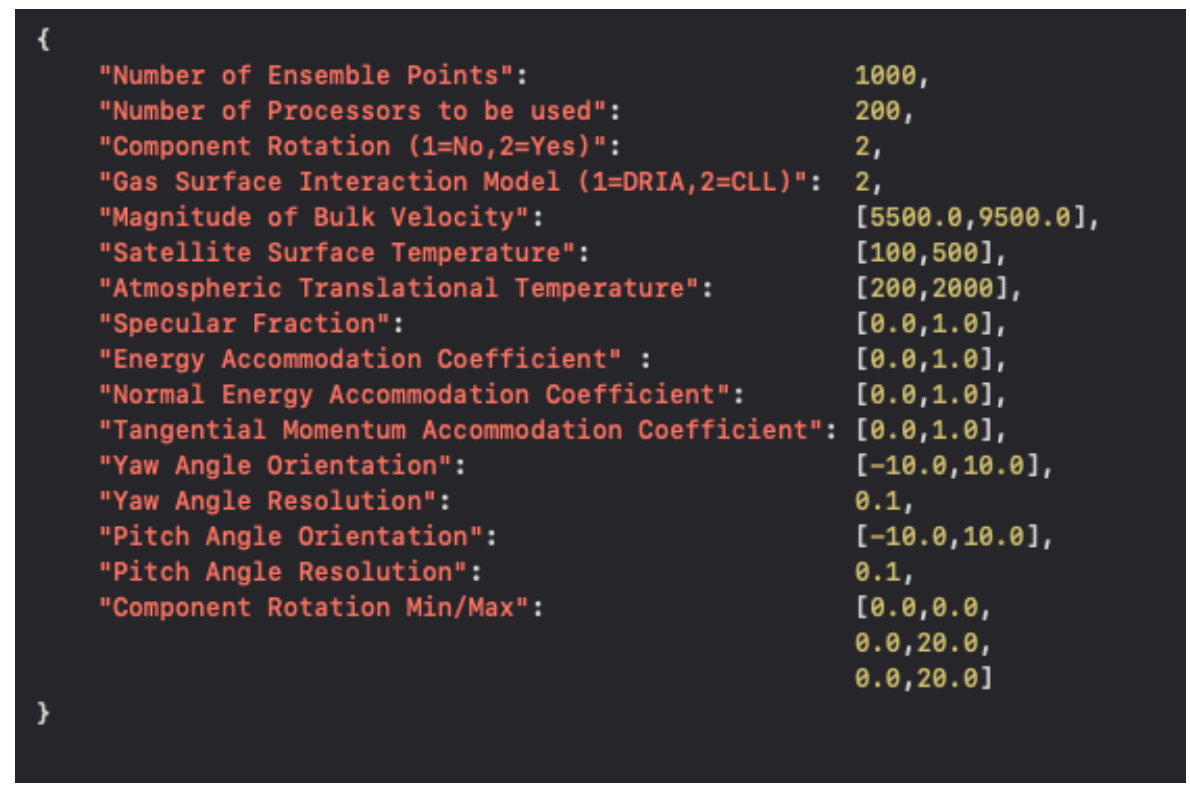

Figure 3.6: Example of the Simulation.json file

A description of the inputs are as follows:

1. Object Name: This is the name that will be given for the regression model. If not performing rotation, the filename of the STL must be used.

2. Number of Ensemble Points: This is how many simulations will be performed by TPMC to create the regression model.

3. Number of Processors to be used: processors used to run simulation in parallel.

4. Component Rotation: A flag to tell the software if the user wishes to have components of the satellite rotate.

5. Gas Surface Interaction Model: Flag for user to define GSI model. 
6. Magnitude of Bulk Velocity: Min/Max value of Velocity sampling; in meters per second.

7. Satellite Surface Temperature: Min/Max value of Surface Temperature sampling; in Kelvin.

8. Atmospheric Translational Temperature: Min/Max value of Atmospheric Temperature sampling; in Kelvin.

9. Specular Fraction: Min/Max value of Specular Fraction; unitless.

10. Energy Accommodation Coefficient: Min/Max value of EAC; unitless.

11. Normal Energy Accommodation Coefficient: Min/Max value of normal EAC; unitless.

12. Tangential Momentum Accommodation Coefficient: Min/Max value of momentum accommodation coefficient; unitless.

13. Yaw Angle Orientation: Min/Max value of yaw sampling; in degrees.

14. Pitch Angle Orientation: Min/Max value of pitch sampling; in degrees.

15. Component Rotation Min/Max: An array for the Min/Max value of each component being rotated. Each row is a component that corresponds to the order of parent.txt; in degrees.

Formatting of the JSON file is very important. The file requires opening and closing brackets and commas after every value except the last one. The "Min/Max" inputs need to be in brackets and the component rotation array has commas after each number except the last value. This array was put in this form for aesthetic reasons. The number of rows should match the number of components. If the user desires to not rotate a certain component, zeros for the maximum and minimum value should be used. To reiterate, if the user is not performing a com- 
ponent rotation, the filename of the mesh for the "Object Name" input should be used. For example:"Object Name": "Satellite.stl" would be the appropriate input for an object not using component rotation.

\subsubsection{STL_FILES}

This folder is where the user should place their STL files, components and whole structures alike.

\subsubsection{STL_ROTATION_INPUTS}

There are three important files in this folder; hinge_points1.txt, hinge_points2.txt, and parents.txt. Each of these is a text file that needs to be altered with the evaluation of new structures and components. The user needs a high level of understanding of the structure of their object for this, as the rotation of each component is determined by a user defined hinge line, and the individual components are combined to form a singe structure for simulation.

To start, the parents.txt file is how the user defines the component STL file being used. The order of the files is very important here. The order the files are listed in this text file dictates the order of the hinge point definitions as well as the order of the "Min/Max" of the component rotation definitions in the Simulation.json file.

The hinge point files define the line of rotation for each component. The files are structured using an $x-y-z$ Cartesian coordinate system where each row is a point corresponding to the component in the same row of parents.txt. Each of the two files define a point in space that corresponds to the other file. For instance, hinge_points1.txt's first row corresponds to hinge_points2.txt's first 
row. Theses two points draw a vector about which the component rotates. The line follows the right hand rule. Going from point 1 to point 2 , if the user were to point their right thumb in the direction of this vector and curl their fingers, that curl would denote the positive direction of rotation for that component. Correlation and examples of the files are shown in Figure 3.7

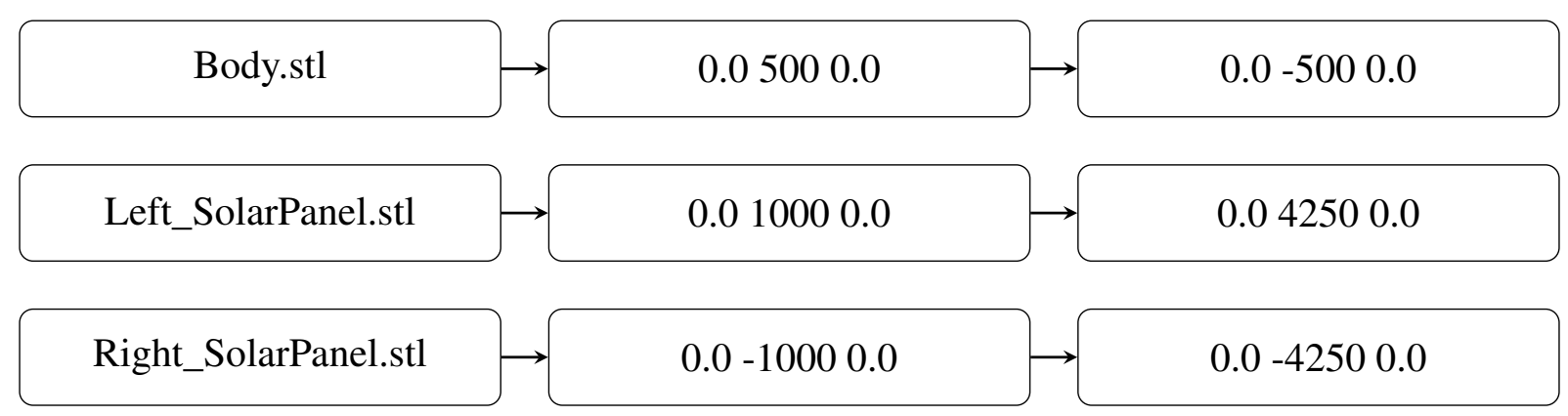

Figure 3.7: Correlation between STL_Rotation_Input Files
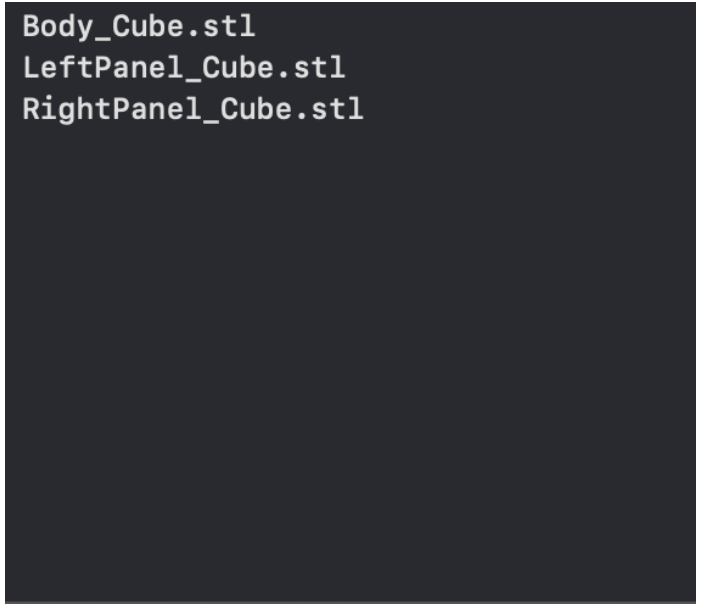

Figure 3.8: Example of the parent.txt file 


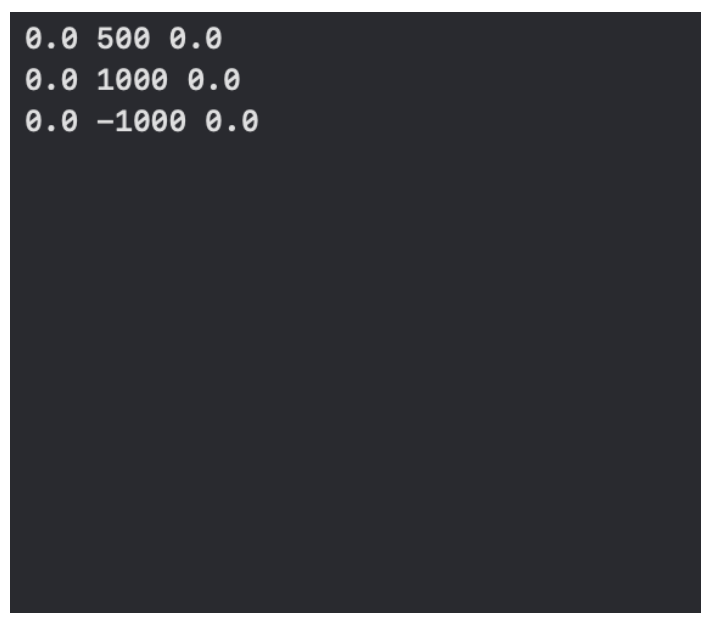

Figure 3.9: Example of the hinge_points1.txt file

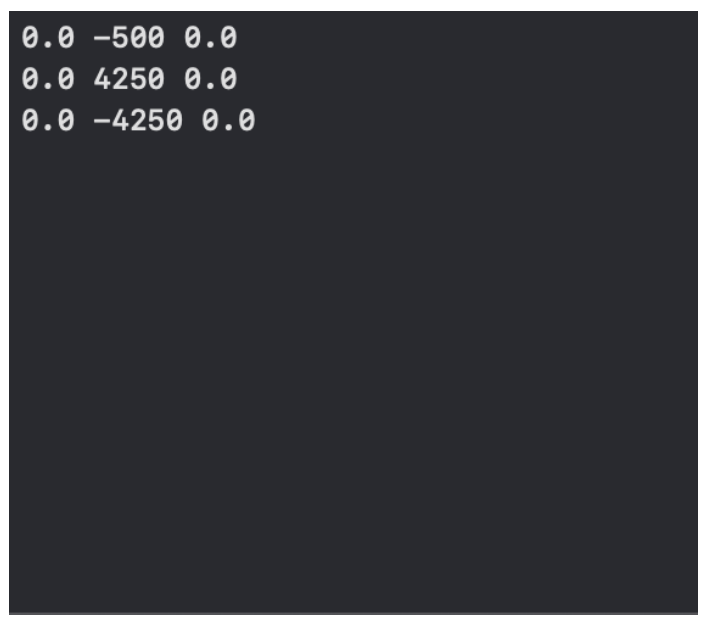

Figure 3.10: Example of the hinge_points2.txt file

\subsection{RSM CODE EXECUTION}

Executing the code is a relatively simple process with few steps. Once the user has loaded their modules and defined their inputs, all that remains is to execute the software. Code execution must be done from the top level directory to ensure there are no issues with file navigation within the code. To execute, the user inputs the following line: 
\$./RSM_TPMC/rsm_run_script.py - -tpm I

RSM_TPMC/tpm/build/src/tpm

A condition with this execution line is that the path of the tpm code needs to be defined. The path to the compiled code is stated after the --tpm flag because the user places the compiled code in their desired and personally defined path. Once the code begins the user will see the Response Surface Model logo along with several print statements. Depending on the mesh of the STL file, the number of simulations being performed, and the number of processors being used, the completion of simulations can take anywhere from a matter of hours to several days. A very complex satellite with a fine mesh can even take several weeks to complete.

\subsection{OUTPuts}

There are two main locations where information is output in the RSM: Outputs and tempfiles. The information and data from the TPMC simulations, as well as the regression model creation, are stored in the Outputs folder for the users convenience. The outputs that the user does not need to interact with are placed in the tempfiles folder.

\subsubsection{TPMC Simulation OUTPUTS}

The tpm.c code creates several outputs. First, it creates training and test data for the regression model. With six species of gas, there will be six training and six test files. These twelve files are placed in the following directory: /Outputs/RSM_Regression_Models/data The test and training data reflect the variables 
created from Latin Hypercube Sampling for each simulation as well as the calculated drag coefficient.

Furthermore, the tpm.c code outputs the projected area of each simulation. The area is saved to the Aout.dat file located in the ./Outputs/Projected_area directory. This file is then used by the area lookup code to calculate the projected area given by the user inputs.

\subsubsection{REGRESSION MODEL}

The regression model code uses the training and test data files to create and test the model. Verification of successful model creation is shown through several plots placed in the following directory: /Outputs/RSM_Regression_Models/Plots_Output folder. Figure 3.11 is an example of the $\mathrm{O} 2$ model created for a simple cube.

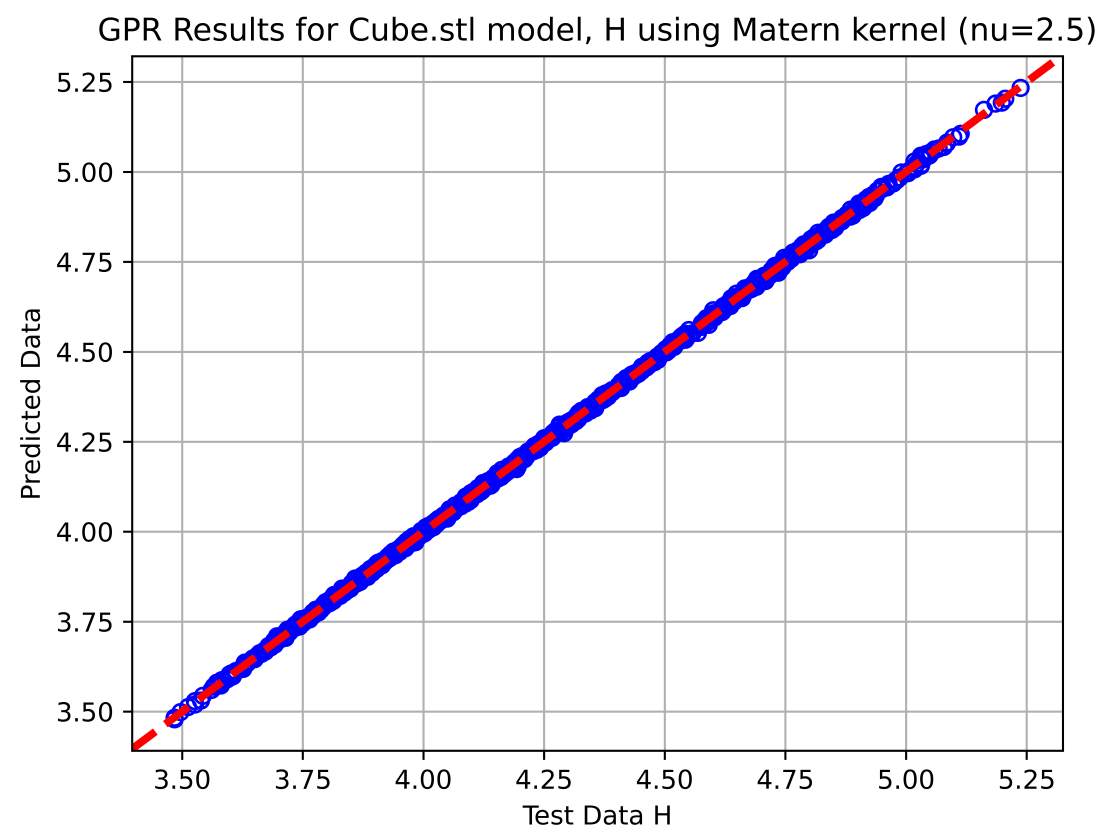

Figure 3.11: Visualization of Model Performance 
The regression model has a lot of outputs that the user will need to interact with as well. Once the model is created the appropriate outputs are placed in the /Outputs/RSM_Regression_Models folder. There are thirty files created from this process in total, each sharing the object name used in the Simulation.json file. Each species requires two files for mean and standard deviation, as well as a Gaussian Process Matern model. The only interaction the user must perform with these files is to move them to the ./Inputs/RSM_Regression_Models folder in order for the model evaluation script to use the correct regression model.

\subsubsection{Model Evaluation Outputs}

After the user executes the model evaluation code, there are only two outputs: the drag coefficient and the projected area. These are named according to the Model_Evaluation_Inputs.json file. Drag coefficient is placed in the

Cd_Results folder and projected area is placed in the Projected_Area folder. These are the final results for the user. They can be visualized through plotting and/or used for orbit propagation as the user sees fit.

\subsubsection{LATIN HyPERCUBE SAMPLING}

Latin Hypercube Sampling is used to make randomized data points for the TPMC simulation. In the tempfiles folder, two files are created; tpm.ensemble and tpm.ensemble.h5. These files are the same but the HDF5 file is used particularly for the metadata included in the file type. The HDF5 is not visible without using the $\$$ h5dump command or an HDF5 viewer. The user can easily view the tpm.ensemble file with any common text editor or reader though. If the user wishes to see the the deflections of each component further they may reference 
the deflections.txt file in the Outputs folder. This file contains similar information as the ensemble files; however this structure is more intuitive. The order of this file also correlates to the order of the mesh file created during rotation. For instance, if the first mesh file created was Satellite0.stl and the first line of deflections.txt was "10 20 30", opening and physically viewing the STL would show the first component rotated 10 degrees, the second rotated 20 degrees, and the third rotated 30 degrees.

\subsubsection{MESH_FILES}

As the rotation script creates STL files for the tpm.c code, they are placed in the Mesh_Files folder. The folder is not automatically cleaned after the software is finished. For now, the files are not deleted so the user can view the STLs if they wish to do so. Since a multitude of STLs are created for a large number of simulations, the user should clean this folder periodically. The user also does not need to interact with these files. Each one is checked for water-tightness as it is created. If there are any issues, the code will automatically exit and notify the user of the error.

\subsubsection{TEMP_VARIABLES.TXT}

temp_variables.txt is used to essentially pass variables from the Python code to $\mathrm{C}$ code. The variables that are passed are the following: Object Name, Gas Surface Interaction flag, Species Mole Fraction, and the Component Rotation flag. The user does not need to interact with these and changing them manually will have no effect on how the software operates. 


\subsection{Model Evaluation}

Evaluating the model is the pinnacle of the user obtaining results. The user has two action items to perform before being able to do so. First, there are the three input items to execute the evaluation. Once these are set, the user needs to execute the code similarly to the simulation execution. Secondly, the two outputs generated from the RSM simulation code need to be placed in the Inputs folder.

\subsubsection{Model Evaluation InPUts}

Under the Inputs folder, there are three inputs that need to be set before execution: the JSON file, the CSV file and the regression models. The regression model is a simple setup. The user must take the regression model created in the simulation execution steps and move them to the following directory: ./Inputs/Model_Evaluation_Inputs/Regression_Models

Model_Evaluation.json is similar to the Simulation.json file. The structure requires the same JSON formatting as before; however, there are no arrays in this file. This is worth noting as the user should be wary that the last value has no comma after it. Figure 3.12 is an example of the file. 


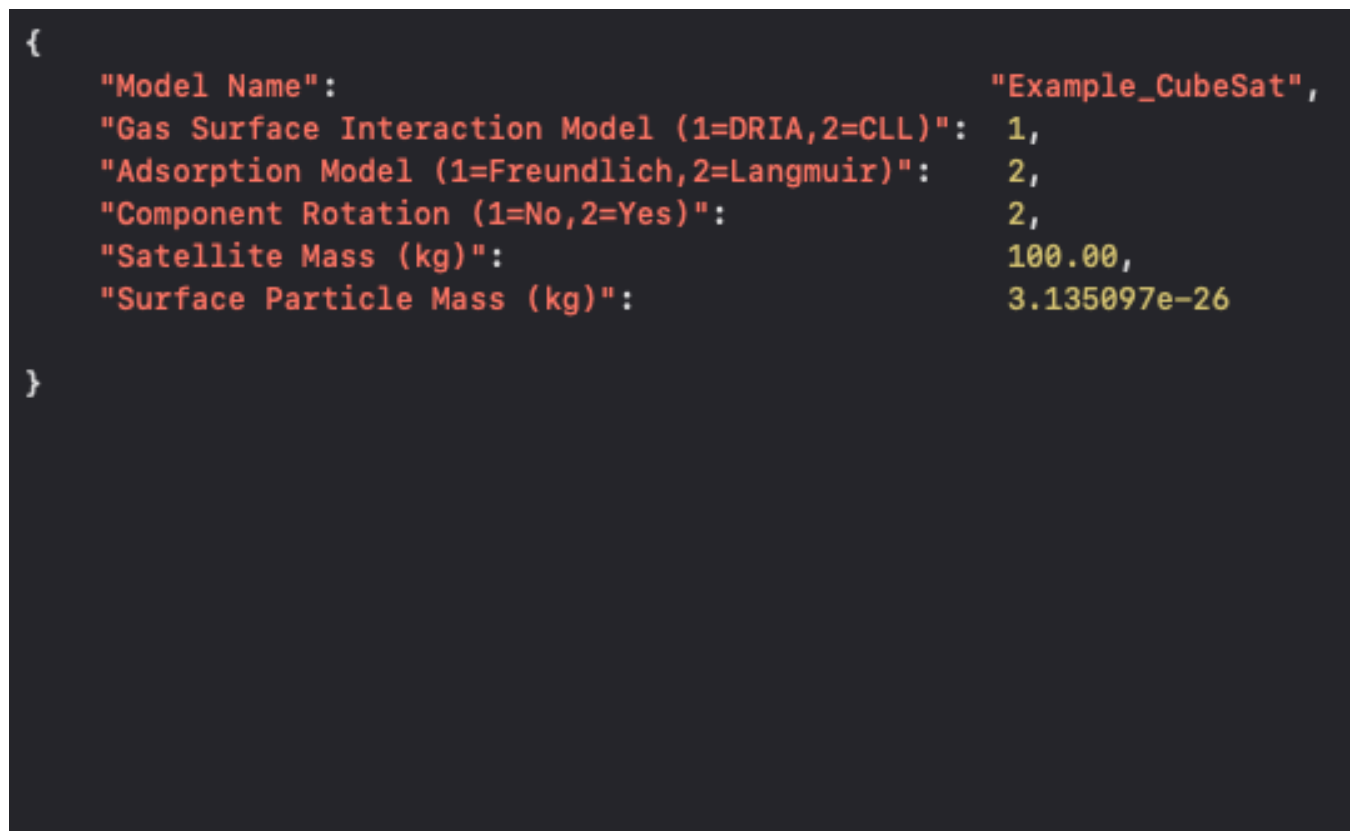

Figure 3.12: Example of the Model_Evaluation_Inputs.json file

The first input of this file is the "Model Name". This is not a name the user is assigning to anything. This is the model name that precedes all of the regression model files. For example, if the user had regression models name Fun_wTPMC_GP_reg_H_matern2.pkl, Fun_wTPMC_x_train_H_mean.pkl, Fun_wTPMC_y_train_O2_mean.pkl, etc. then "Model Name": "Fun_wTPMC" would be the appropriate input. If the user performed simulation without rotation, then the caveat of using the .stl extension carries over to this input file. For example, the user could have an input such as "Model Name": "Satellite.stl".

The next input is the GSI model. This must correlate with the GSI used when the simulation was preformed. If these do not match then the program will throw an error. Similarly, the Component Rotation input here must also match what was used for the model. These two inputs are important because they determine the number of variables as well as the number of dimensions used, respectively.

The adsorption model input is set at the users discretion. Using either of these 
determines how the fractional surface coverage of adsorbed oxygen is perceived. A snippet of the code that calculates the fractional surface coverage is shown in Figure 3.13

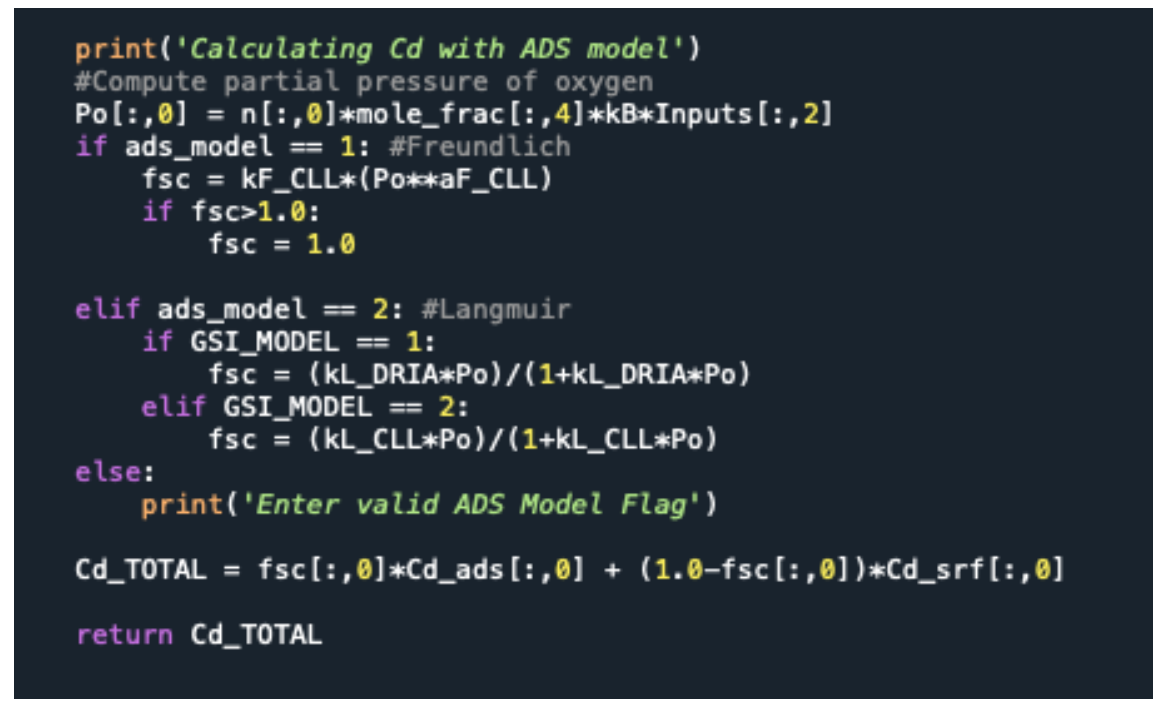

Figure 3.13: Code from model_evaluation_script.py, calculation of the fractional surface coverage of adsorbed oxygen with different ADS models.

The last input is the satellite surface particle mass. The surface particle mass is used for the calculation of the energy accommodation coefficient for clean surfaces based on Goodman's (1966) empirical formula. This is a value that can vary from satellite to satellite and is dependent of the satellite's material and finishing.

Model_Input_Data.csv is where the user may input as many data points as they desire to find the drag coefficient at any given instance. A CSV file was chosen to allow the flexibility of inputting a single instance, multiple instances, or time series data as well as the ability to allow for n-dimensions from the component rotation. Each column of the file is an input and denoted in the header of the file. The inputs are as follows: 
- Velocity $(\mathrm{m} / \mathrm{s})$

- Surface Temperature (K)

- Atmospheric Temperature (K)

- Yaw (radians)

- Pitch (radians)

- Number Density ( $\# / m^{3}$, the number of particles per meter cubed)

- Mole Fraction (H)

- Mole Fraction (He)

- Mole Fraction (N)

- Mole Fraction (N2)

- Mole Fraction (O)

- Mole Fraction (O2)

- Component Rotation... (degrees)

Each row of the file is considered to be one instance of evaluation. The CSV file requires that each of these columns remain in this particular order also. The component rotation flag that is used in the Model_Evaluation_Inputs.json tells the code if the columns after "Mole Fraction $(\mathrm{O} 2)$ " are relevant or not. If the flag is turned off then these columns will not be read. It is important that the number of "Component Rotation..." columns matches the number of components being used in the simulations. All of the inputs prior to the component rotation inputs are used to calculate the drag coefficient of the satellite. The component rotation inputs themselves are relevant to find the projected area of the satellite. 


\subsubsection{Execution of Model Evaluation}

Executing the model evaluation script is similar to the RSM run script. A difference is that the evaluation does not require any $\mathrm{C}$ code. Therefore, a simple Python execution is necessary. Similar to other code executions, the call must be made from the top level directory.

\section{\$./RSM_TPMC/model_evaluation_script.py}

\subsubsection{Outputs of Model Evaluation}

There are two outputs to the model evaluation: the drag coefficient and the projected area of the satellite. Each of them are placed in the Outputs folder in their respective folders; $C d \_$Results and Projected_Area. The output files are text files with the output printed as a column of data in the same order as the CSV file used at input.

\section{Results}

\subsection{Check Water-Tightness}

The check water-tightness function created was successful in preventing erroneous STL files from being tested. The function was able to find very minute discrepancies in multiple STL files during testing. An example of the the function in operation is shown in Figure 4.1 


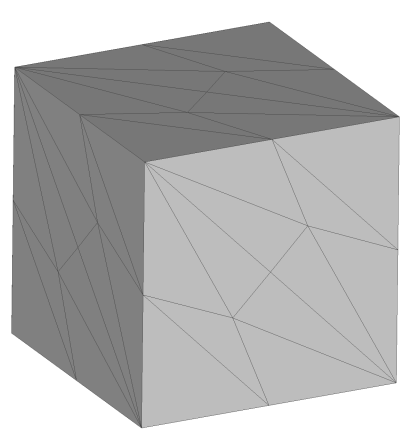

(a) Whole Cube

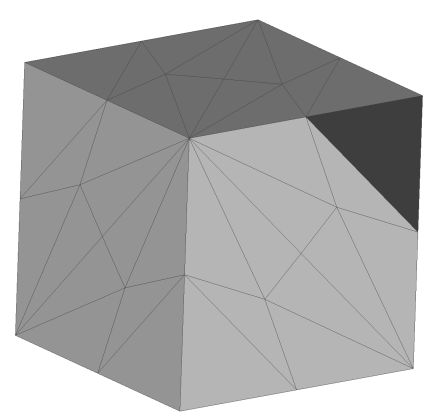

(c) Broken Cube

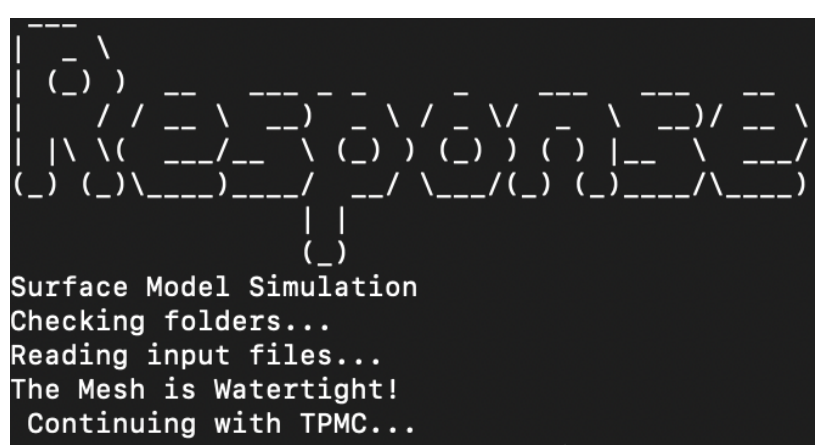

(b) CWT Results - Pass

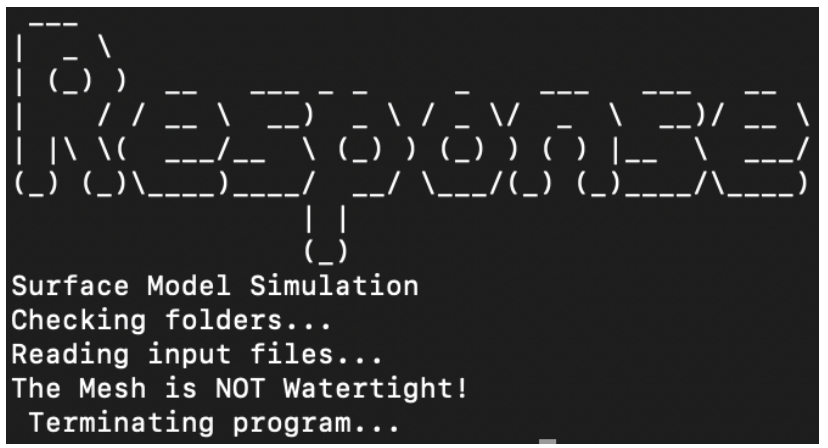

(d) CWT Results - Fail

Figure 4.1: CWT Results for a Whole Structure and a Broken Structure

Figure 4.1 shows a cube with a single facet removed. With the facet removed, the program terminates and gives the appropriate pass-fail message. In the case of rotation being performed, with new STL files being automatically generated, each new STL file is also ran through the CWT function. The function itself is very efficient and only adds additional milliseconds for each CWT run. The CWT for rotation also gives the user information about which configuration of the STL passes/fails. An example of the CWT with rotation is given underneath the "Example of the WVU RSM Toolkit Operation" section of this work in 
Appendix A.

\subsection{Comparison of Closed Form Solutions}

Several closed form solutions for simple shapes exist that may serve as a baseline for the performance of the RSM. The three shapes evaluated are the cube, sphere, and cylinder. Each is a function of the molecular speed ratio given by Equation 4.2. The molecular speed ratio is the ratio of the freestream velocity, $U$, to the relative gas velocity, $v_{m p}$. The relative gas velocity is more precisely defined as the most probable speed of a Maxwellian velocity distribution at the local translational temperature of the atmosphere and is defined by Equation 4.1 [37]. The velocity distribution is comprised of the Boltzmann Constant, $k_{b}$, atmospheric temperature, $T_{\infty}$, and the mass of one atom of oxygen in kilograms, $m$.

$$
\begin{gathered}
v_{m p}=\sqrt{\frac{2 k_{b} T_{\infty}}{m}} \\
s=\frac{U}{v_{m p}}
\end{gathered}
$$

To start, the closed form solution of a cube can be given by Equation 4.3 .

$$
C_{D, c u b e}=\frac{2}{\sqrt{\pi} s} e^{-s^{2}}+\left(2+\frac{1}{s^{2}}\right) \operatorname{erf}(s)+\frac{\sqrt{\pi}}{s} \sqrt{\frac{T_{s}}{T_{\infty}}}+\frac{4}{\sqrt{\pi} s}
$$

Once the closed form solution has been created, it is possible to compare the LANL RSM and WVU RSM results with the solution. Comparison of the closed 
form solution, the LANL RSM, and the WVU RSM, are shown in Figure 4.2 and Figure 4.3 . Figure 4.2 s results are obtained with a variation of freestream velocity from $5500 \mathrm{~m} / \mathrm{s}$ to $9500 \mathrm{~m} / \mathrm{s}$, a satellite surface temperature of $300 \mathrm{~K}$, an atmospheric temperature of $1100 \mathrm{~K}$, and complete accommodation. In Figure 4.3, the velocity was kept at $5500 \mathrm{~m} / \mathrm{s}$, with the surface temperature at $300 \mathrm{~K}$, and complete accommodation. The LANL RSM and WVU RSM toolkits have high fidelity outputs in both cases. Both regression models are also proven to find solutions that are reasonably close to the closed form solution. Furthermore, between the two models, the WVU RSM model has shown that it can provide the same level of accuracy as the LANL RSM regression model.

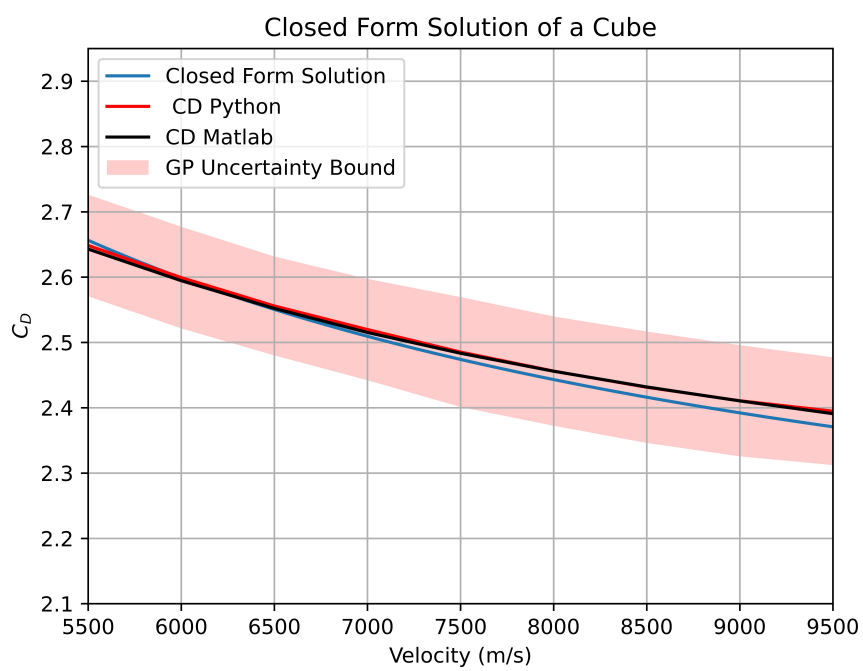

Figure 4.2: Closed Form Solution of a Cube with Varying Velocity 


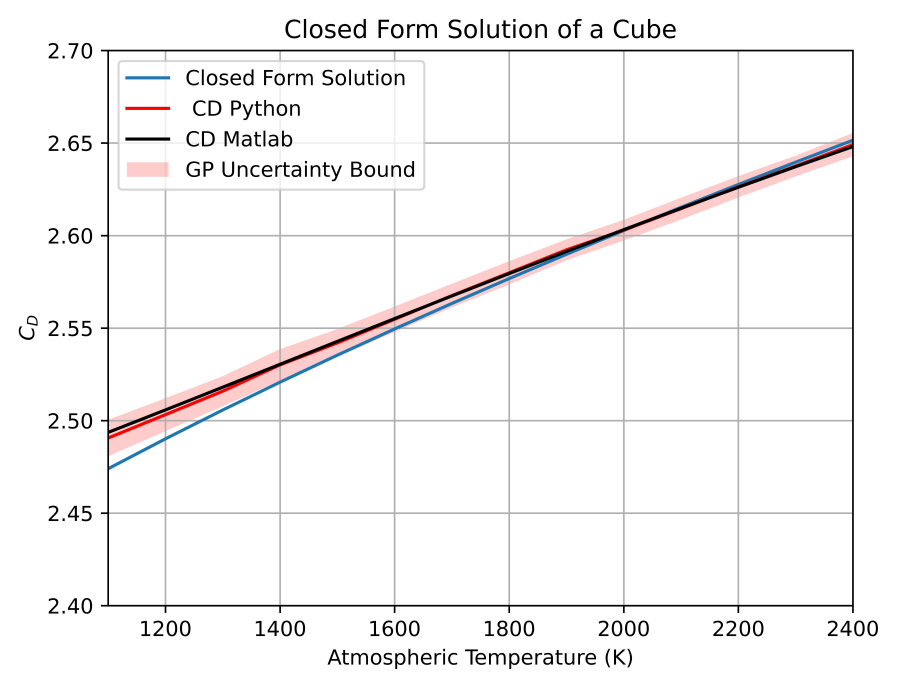

Figure 4.3: Closed Form Solution of a Cube with Varying Atmospheric Temperature

Continuing with further comparison, the closed from solution of a sphere is given by Equation 4.4;

$$
\begin{aligned}
& C_{D, \text { sphere }}=\frac{2-\sigma_{n}+\sigma_{t}}{2}\left(\frac{4 s^{4}+4 s^{2}-1}{2 s^{4}} \operatorname{erf}(s)+\ldots\right. \\
& \left.\frac{2 s^{2}+1}{\sqrt{\pi} s^{3}} e^{-s^{2}}\right)+\frac{2 \sigma_{n} \sqrt{\pi}}{3 s} \sqrt{\frac{T_{s}}{T_{\infty}}}
\end{aligned}
$$

where $\sigma_{n}$ and $\sigma_{t}$ are the normal and tangential momentum coefficients, respectively. A comparison of just the WVU RSM and the closed form solution is given with variation in the velocity, atmospheric temperature, and satellite surface temperature. All three plots show values that are relatively close; however, it can be seen that lower temperatures there is a higher difference between the RSM and the closed form solution. These comparisons were created using nominal values of $U=7500 \mathrm{~m} / \mathrm{s}, T_{\infty}=1100 \mathrm{~K}$, and $T_{s}=300 \mathrm{~K}$ with ranges of $U=$ $[5500,9500] \mathrm{m} / \mathrm{s}, T_{\infty}=[500,2000] \mathrm{K}$, and $T_{s}=[100,500] \mathrm{K}$. 


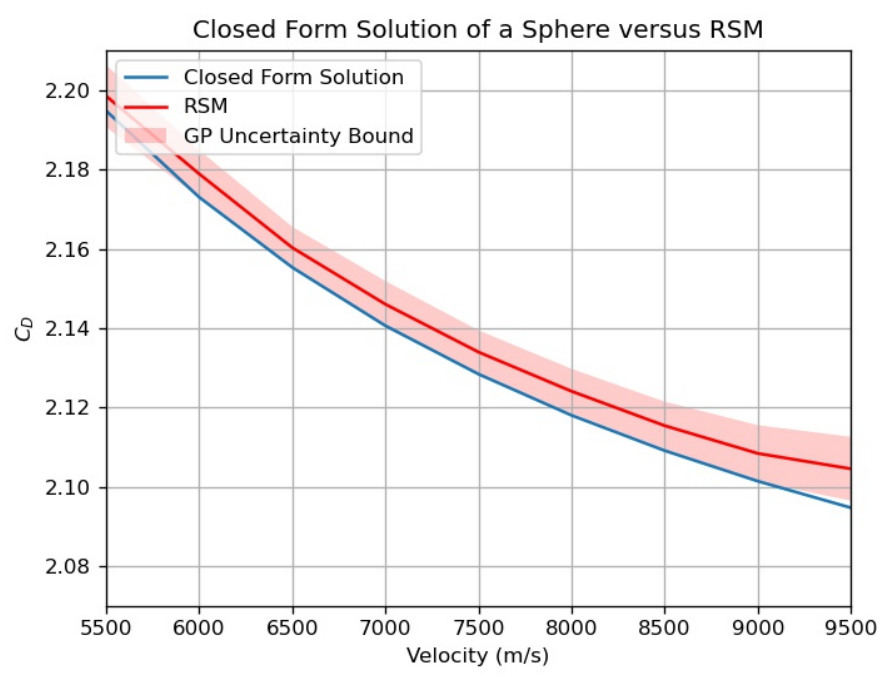

Figure 4.4: Closed Form Solution of a Sphere with Varying Velocity

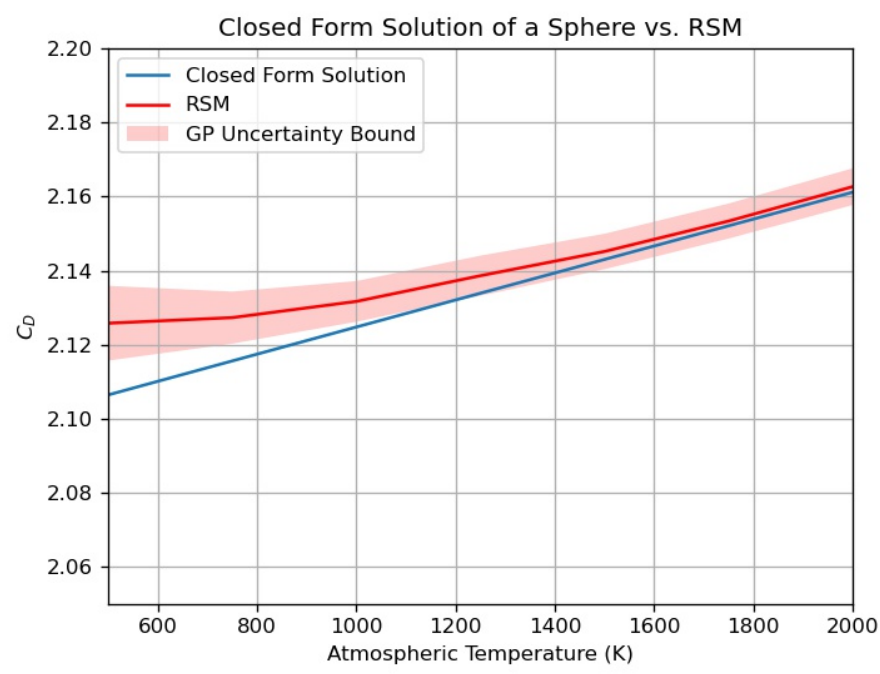

Figure 4.5: Closed Form Solution of a Sphere with Varying Atmospheric Temperature 


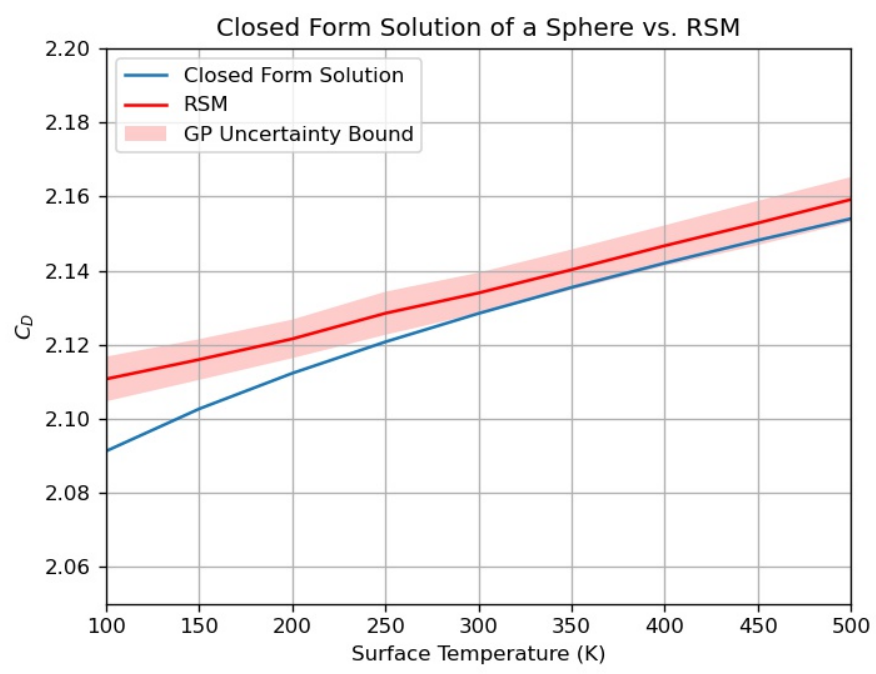

Figure 4.6: Closed Form Solution of a Sphere with Varying Atmospheric Temperature

For one more comparison, the closed form solution of a cylinder with its axis perpendicular to the flow is given by:

$$
\begin{aligned}
& C_{D, \text { cylinder }}=\frac{\sigma_{n} \pi^{\frac{3}{2}}}{4} \sqrt{\frac{T_{s}}{T_{\infty}}}-\frac{\sqrt{\pi}}{6}\left(2 \sigma_{n}-\sigma_{t}-4\right) \frac{e^{\frac{-s^{2}}{2}}}{s} \ldots \\
& {\left[\left(s^{2}+\frac{3}{2}\right) I_{0}\left(\frac{s^{2}}{2}\right)+\left(s^{2}+\frac{1}{2}\right) I_{1}\left(\frac{s^{2}}{2}\right)\right]+\frac{\pi R^{2}}{2 R H} \frac{2 \sigma_{t}}{\sqrt{\pi} s}}
\end{aligned}
$$

where $R$ is the radius of the face of the cylinder, $H$ is the height of the cylinder, and $I_{0}, I_{1}$ are the Modified Bessel Function of the first kind ordered to 0 and 1 respectively. The following figures reflect similar trends as the sphere and cube proving that the RSM is trending correctly with the closed form solutions. 


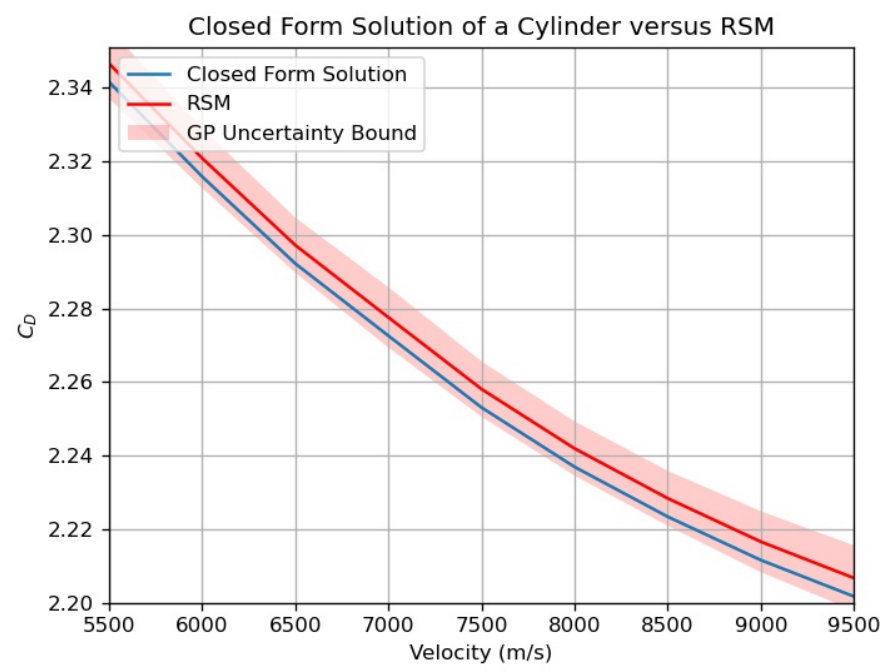

Figure 4.7: Closed Form Solution of a Cylinder with Varying Velocity

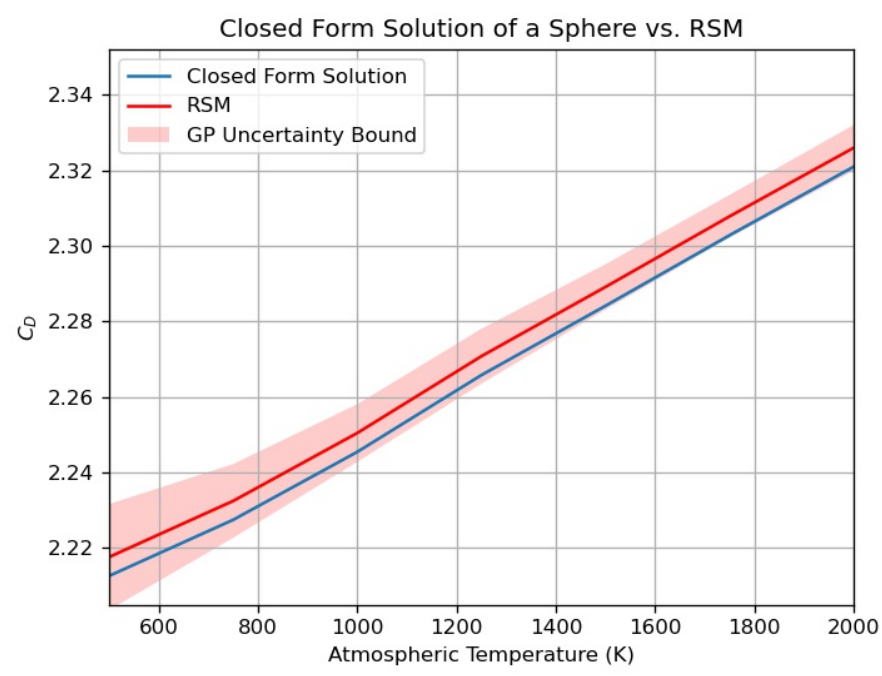

Figure 4.8: Closed Form Solution of a Cylinder with Varying Atmospheric Temperature 


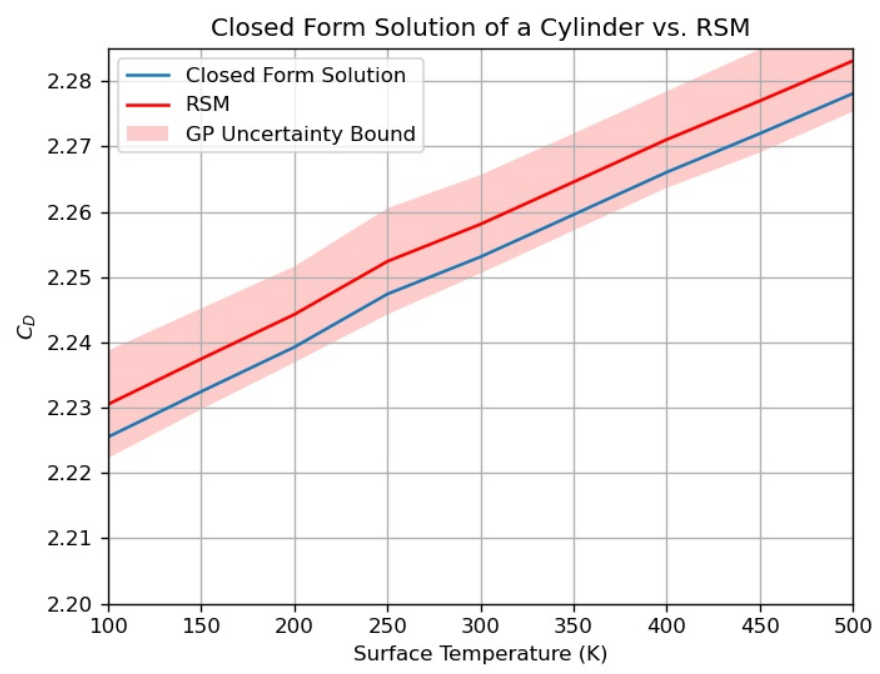

Figure 4.9: Closed Form Solution of a Cylinder with Varying Atmospheric Temperature

\subsection{GaUssian Process RegRession}

\subsubsection{GPR PREDICTION RESULTS}

The GPR's performance is well modeled by the comparison of the test data to prediction values output by the GPR. The plots in Figure 4.10 are representations of the data being compared. The test and prediction data should be near equal and can be seen by the linear trend in the comparison plots. The histograms illustrated in Figure 4.10 represent the residuals of the data being compared. These very low residuals are an indication of excellent GPR performance. Figure 4.10 shows the performance of the GPR for a simple cube with 1,000 ensemble points. 


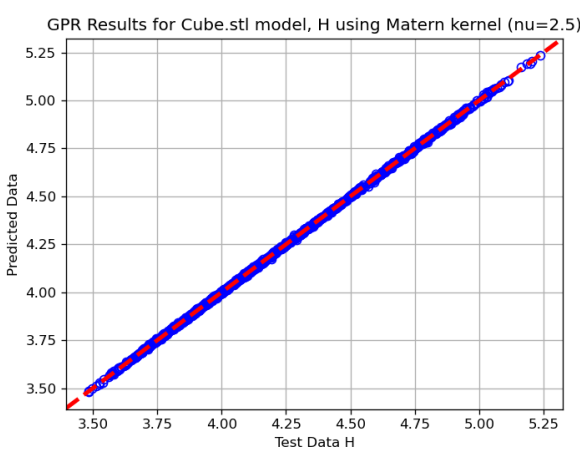

(a) Hydrogen GPR Performance

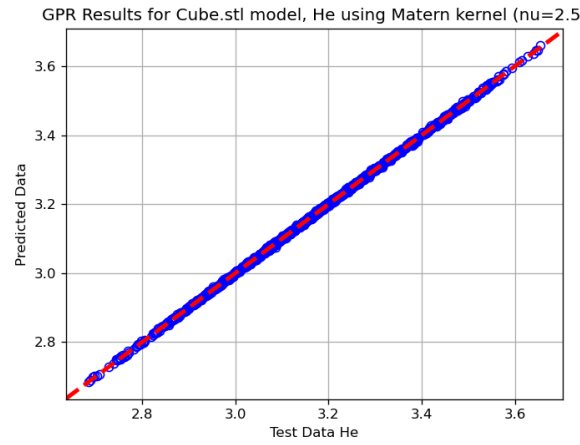

(c) Helium GPR Performance

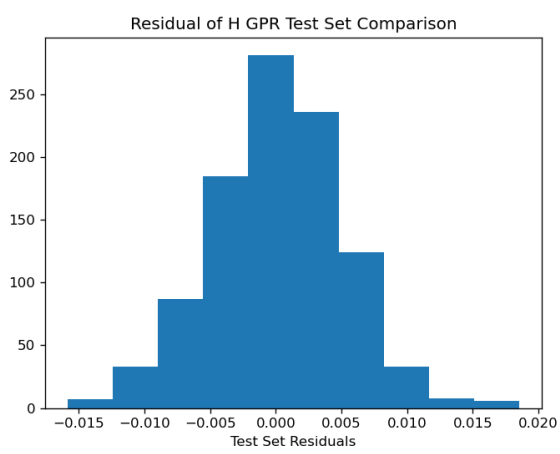

(b) Hydrogen Residuals

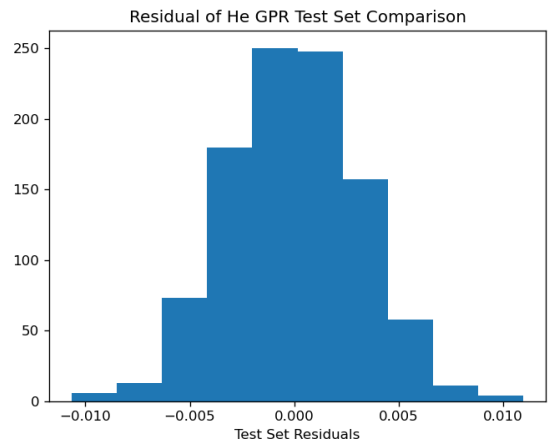

(d) Hydrogen Residuals

Figure 4.10: Performance of GPR with each Species of Gas, Cube 


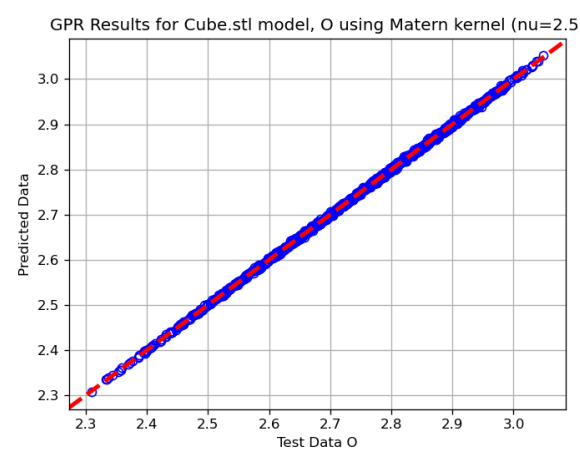

(e) Atomic Oxygen GPR Performance

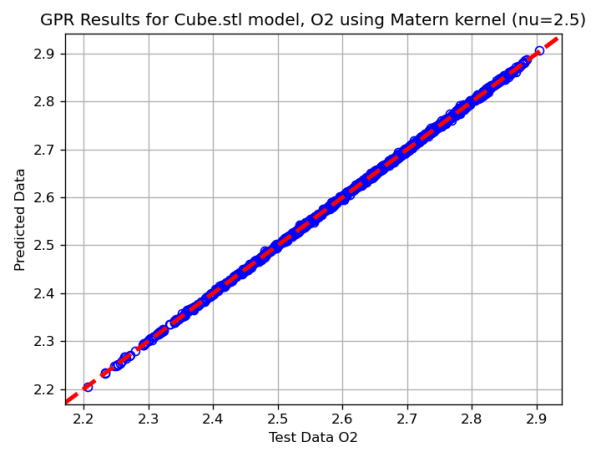

(g) Diatomic Oxygen GPR Performance

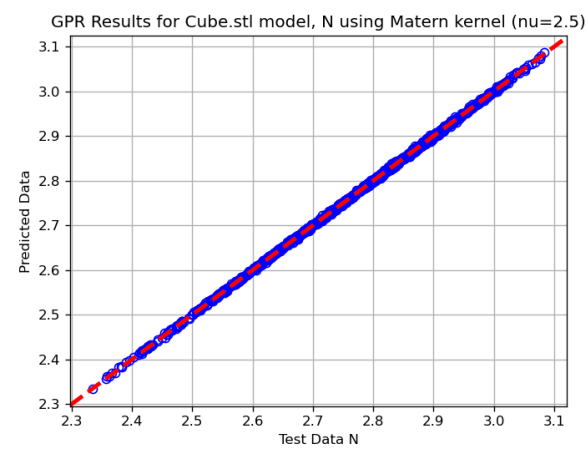

(i) Atomic Nitrogen GPR Performance

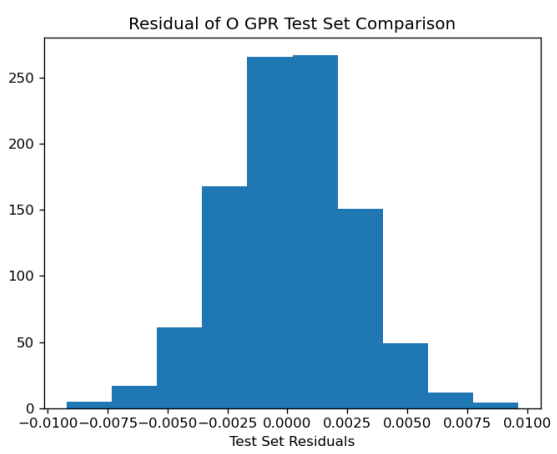

(f) Atomic Oxygen Residuals

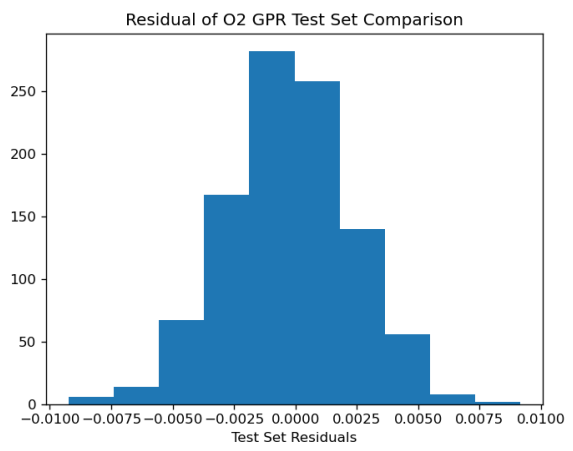

(h) Diatomic Oxygen Residuals

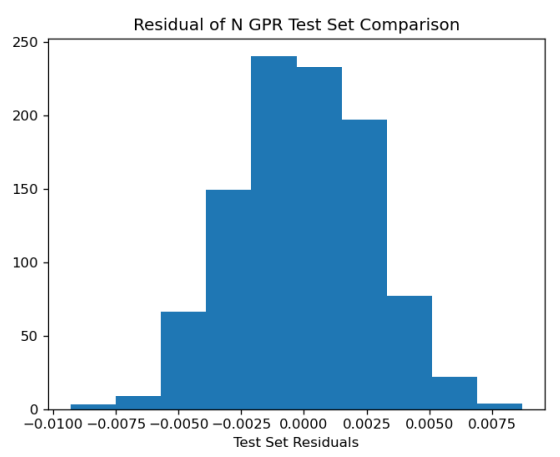

(j) Atomic Nitrogen Residuals

Figure 4.10: Performance of GPR with each Species of Gas, Cube (cont.) 


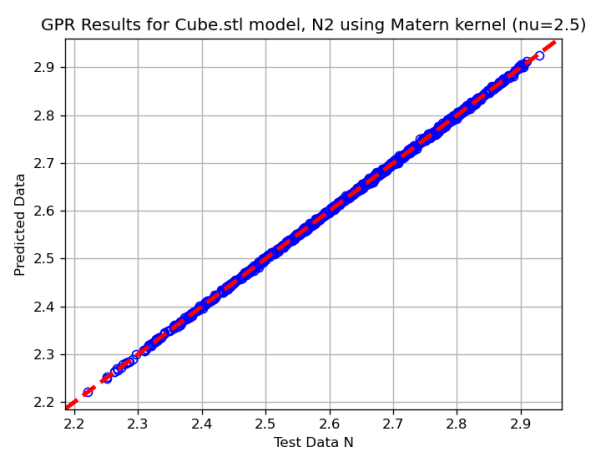

(k) Diatomic Nitrogen GPR Performance

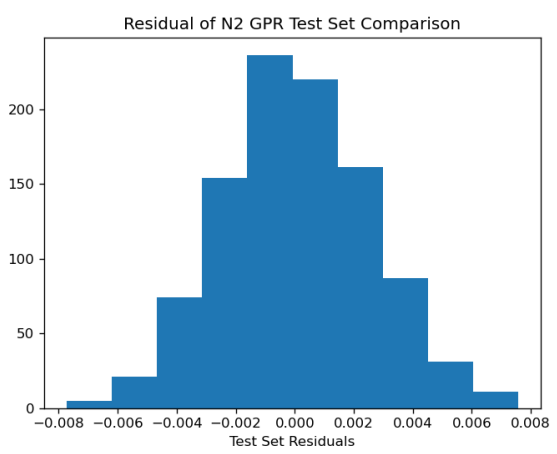

(1) Diatomic Nitrogen Residuals

Figure 4.10: Performance of GPR with each Species of Gas, Cube (cont.)

The evaluation of the cube shows excellent performance. The next evaluation is now of a cube with rotating solar panels. The rotation of each component now becomes an input into the GPR model creation. In this case, the central body of the satellite does not have any rotation other than pitch and yaw. Because of this, the rotation of the central body is set to zero. The code is capable of recognizing this and neglects any input with zero variance. The performance of the GPR with rotation inputs is shown in Figure 4.11. The satellite was evaluated at 1000 ensemble points and also shows excellent performance predicting drag coefficient.

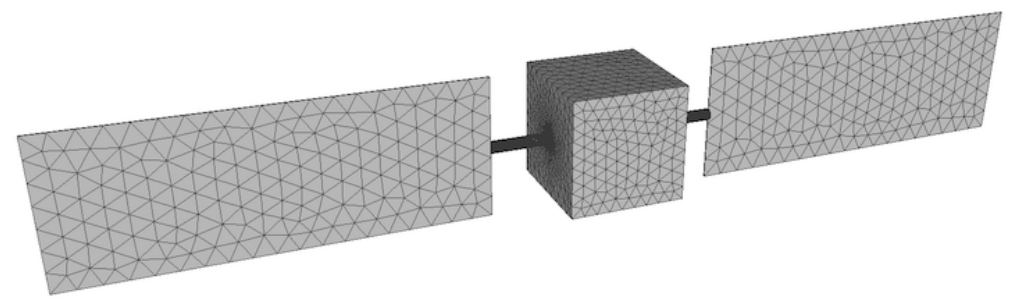

Figure 4.11: Satellite with rotating solar panels 


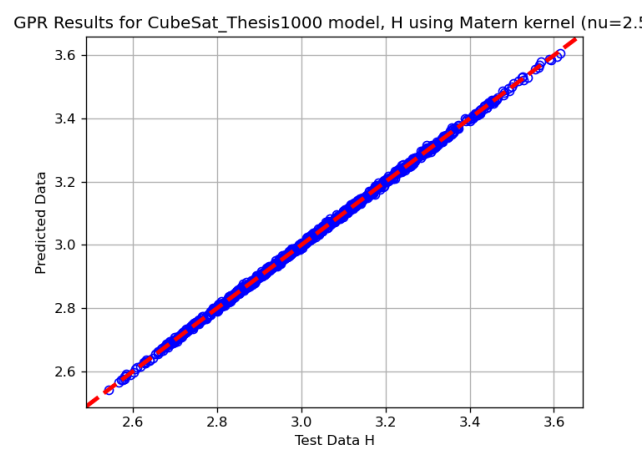

(a) Hydrogen GPR Performance

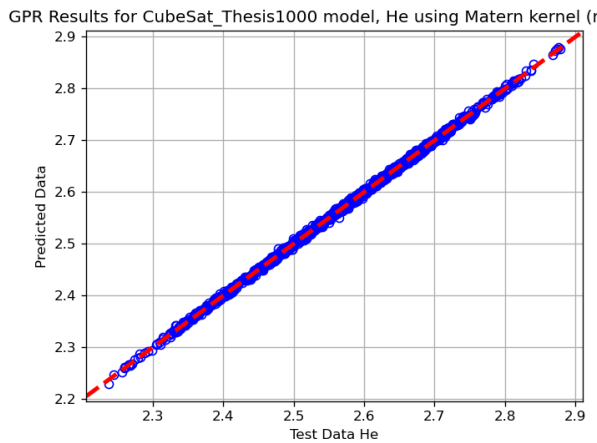

(c) Helium GPR Performance

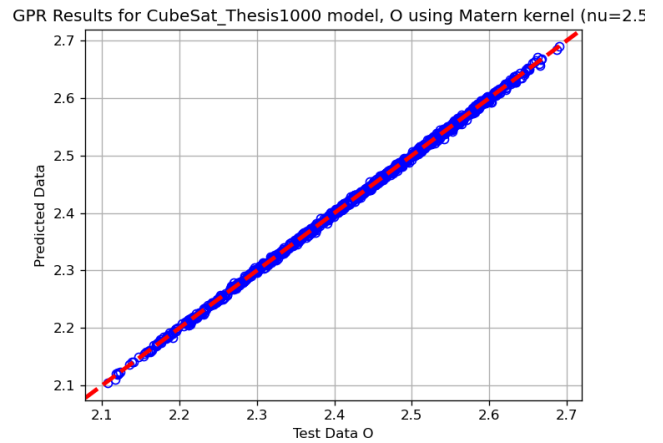

(e) Atomic Oxygen GPR Performance

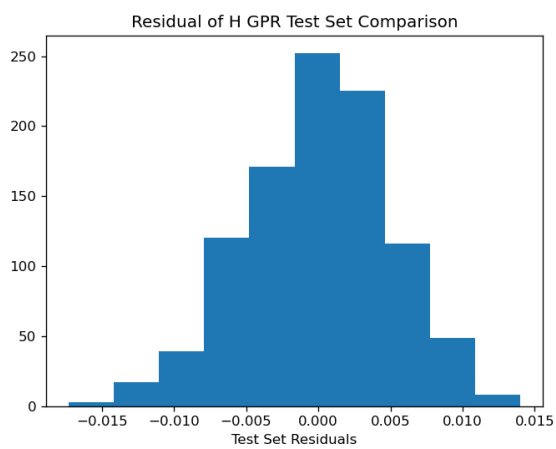

(b) Hydrogen Residuals

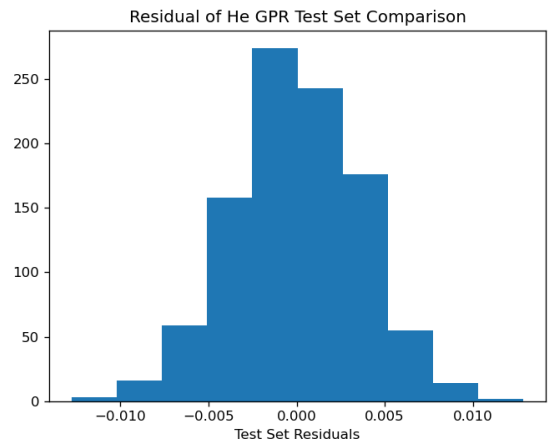

(d) Hydrogen Residuals

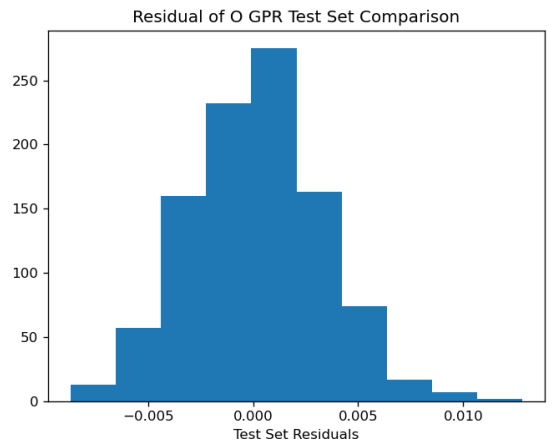

(f) Atomic Oxygen Residuals

Figure 4.12: Performance of GPR with each Species of Gas, CubeSat 


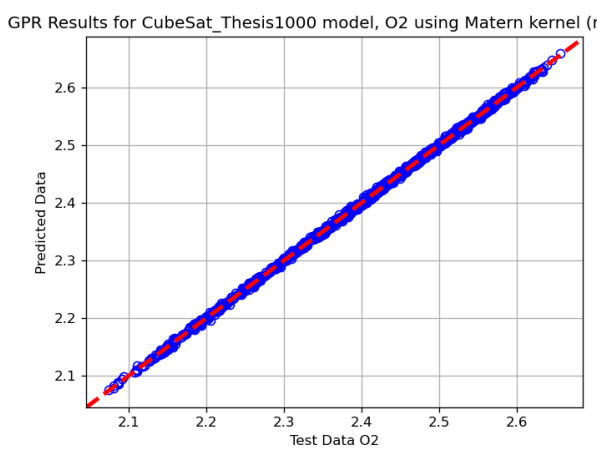

(g) Diatomic Oxygen GPR Performance

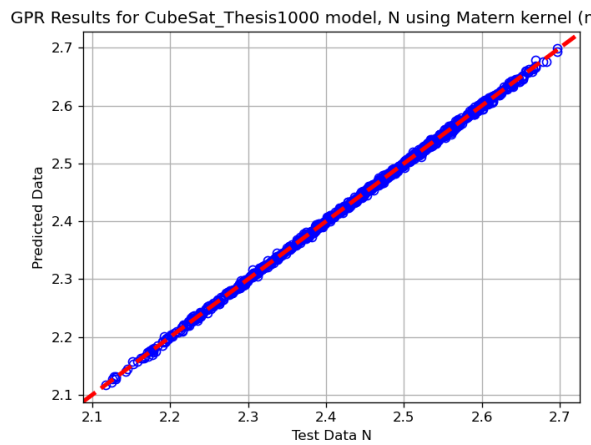

(i) Atomic Nitrogen GPR Performance

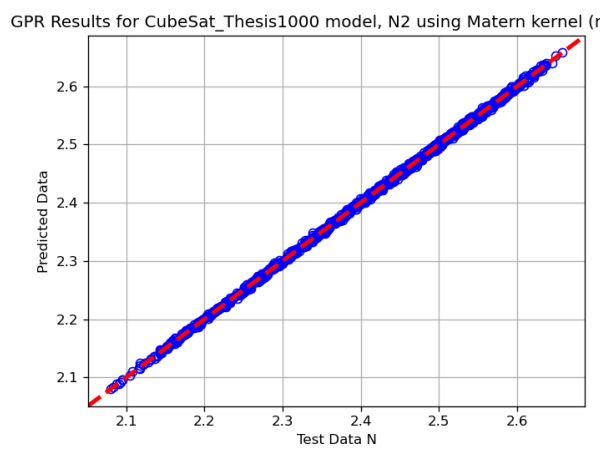

(k) Diatomic Nitrogen GPR Performance

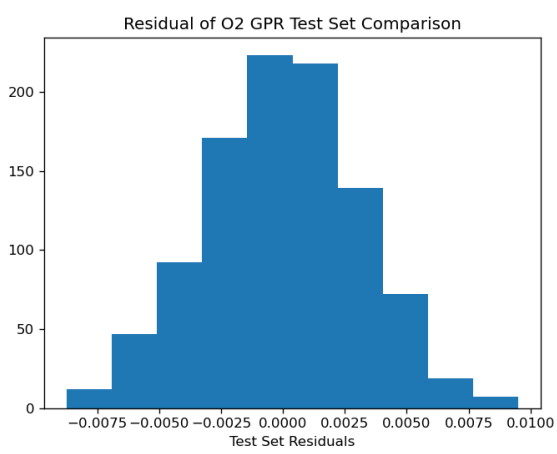

(h) Diatomic Oxygen Residuals

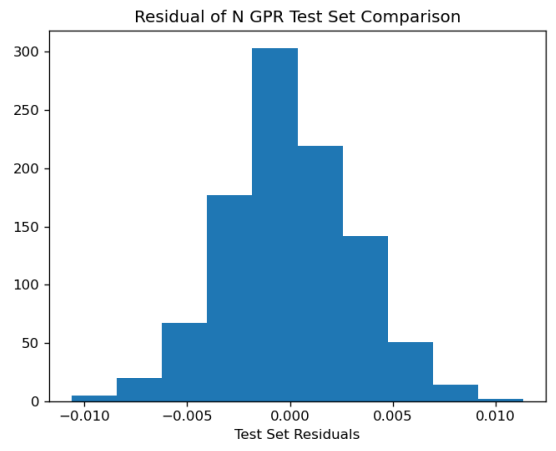

(j) Atomic Nitrogen Residuals

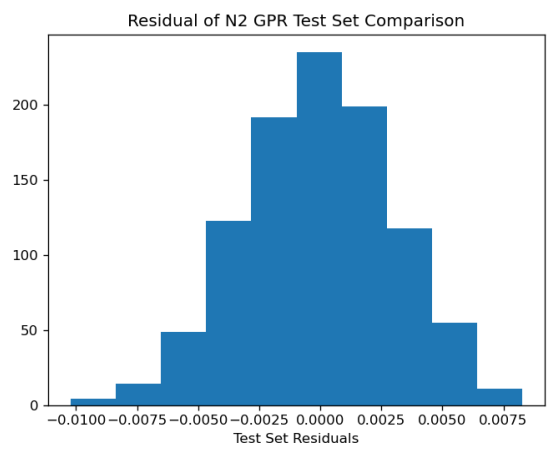

(l) Diatomic Nitrogen Residuals

Figure 4.12: Performance of GPR with each Species of Gas, CubeSat (cont.)

\subsubsection{Model CALibration}

Calibration is the requirement in stochastic modeling that the predicted probabilities give an approximation of the likelihood of true events [6]. A well- 
calibrated model, for example, should have around $68 \%$ true observations within one standard deviation, $95 \%$ true observations within two standard deviations, and $99.7 \%$ true observations within three standard deviations. Uncalibrated models tend to be over-confident or under-confident in their predictions, and one should not trust their inferences. A convenient way to check how well a model is calibrated is by looking at its "consistency curve".

Let the expected confidence interval levels be: $C=[5 \%, 10 \%, 15 \%, \ldots ., 95 \%]$. The corresponding coefficients defining the uncertainty bounds are: $\kappa[j]=$ $\sqrt{2} \operatorname{erf}^{-1}(C[j] / 100)$. Let, $\left(\mathbf{x}_{\mathbf{o}_{\mathbf{j}}}, \mathbf{y}_{\mathbf{o}_{\mathbf{j}}}\right)_{j=1, \ldots, m}$ be the observation dataset and let the corresponding predictions be $\left(\mu_{\mathbf{j}}, \sigma_{\mathbf{j}}\right)_{j=1, \ldots, m}$, where $\mu_{j}$ represents the mean and $\sigma_{j}$ represents the standard deviation. Then, the percentage of observed dataset within the lower and upper uncertainty bounds associated with $C[j]$ is obtained as [10]:

$$
P[j]=\left[\sum_{j=1}^{m} \frac{\mathcal{I}\left(\left(\mu_{j}-\kappa[j] \sigma_{j}\right)<y_{o_{j}}<\left(\mu_{j}+\kappa[j] \sigma_{j}\right)\right)}{m}\right] \times 100
$$

where $\mathcal{I}$ is the indicator function.

The consistency curve mentioned earlier is the plot of $P$ versus $C$. The proximity of the consistency curve to the $y=x$ line (i.e., a straight line with a slope of 450 and passing through the origin) is used to measure calibration in this study. The consistency curve will perfectly overlap the $y=x$ line in a perfectly calibrated system. For the evaluation of consistency, a sphere and the satellite CHAMP are evaluated. CHAMP can be seen in Figure 4.14 and referenced in Section 4.3.3. The consistency plots for sphere and CHAMP drag coefficient predictions using GPR for the test dataset are shown in Figure 4.13, The blue 
dotted line corresponds to the ideal case of perfect calibration, and the green curve corresponds to the GPR predictions. The predictions for the sphere are better calibrated than the predictions for the Champ satellite.

Gaussian Process

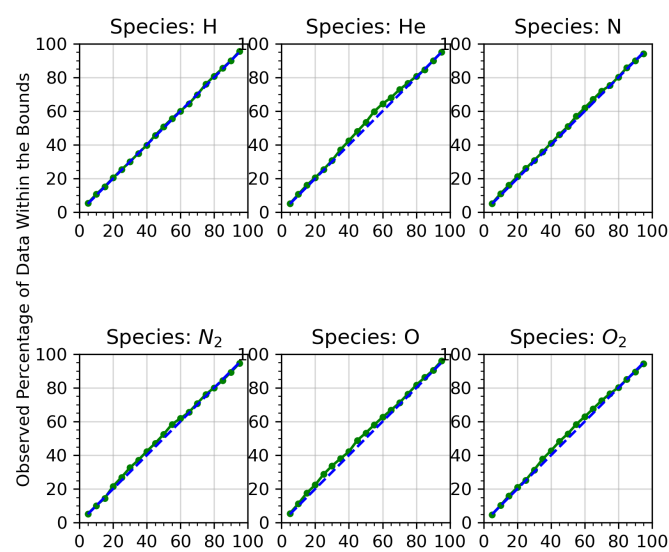

Expected Confidence Level (Percentage)

(m) Sphere Consistency Curve
Gaussian Process

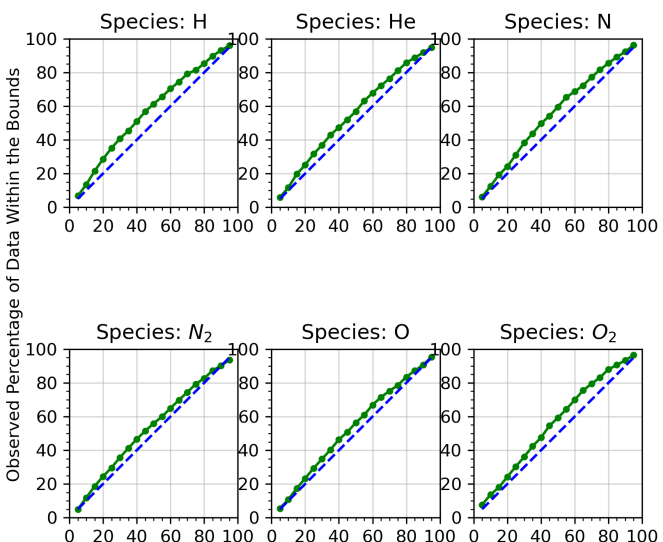

Expected Confidence Level (Percentage)

(n) Champ Consistency Curve

Figure 4.13: Consistency Plots for Sphere and Champ for Drag Coefficient Prediction Using Gaussian Process Regression for the Test Dataset

\subsubsection{COMPUTATIONAL ANALYSIS COMPARISON}

To further test the credibility of the new GPR used in the WVU RSM Toolkit, comparison between several sets of data was performed. Figure 4.15 contains three plots comparing the two codes that use the GPR as well as data provided by Dr. Christian Siemes from the Delft University of Technology [13]. The satellite being evaluated is CHAMP, the "Challenging Minisatellite Payload", a German satellite meant for atmospheric and ionospheric research. The satellite features a relatively complex geometry as seen in Figure 4.14. 


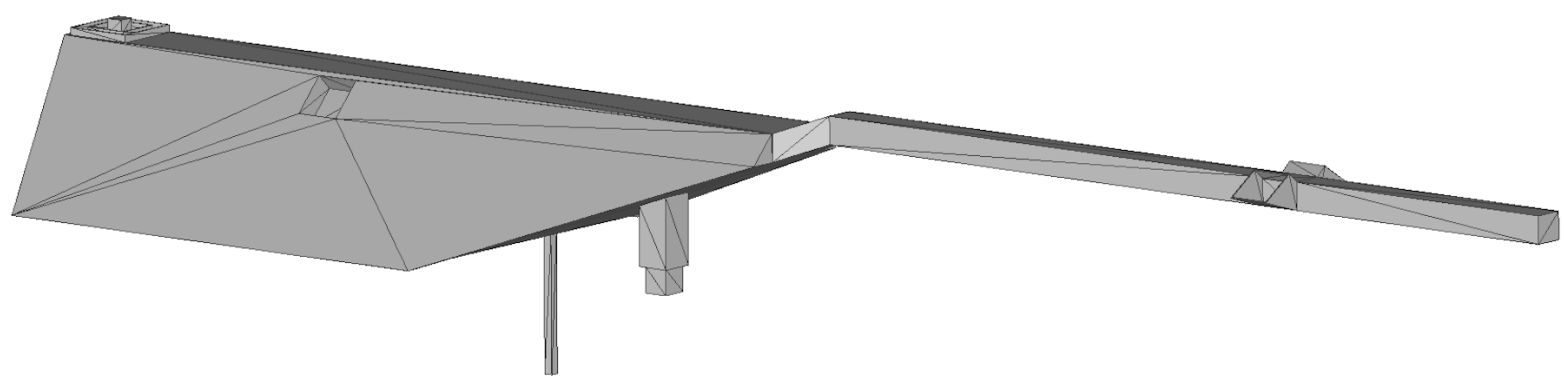

Figure 4.14: CHAMP Satellite 


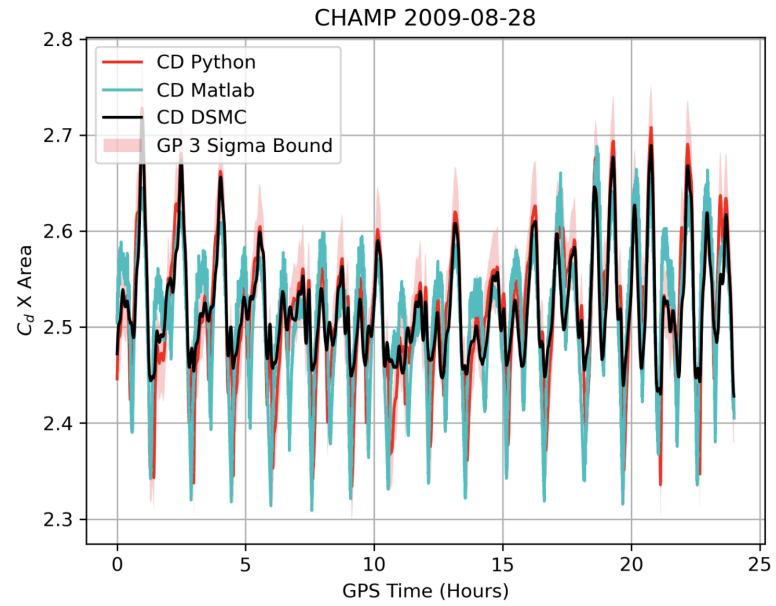

(a) CHAMP 2009 August 28, 24hr $C_{D} \times$ Area

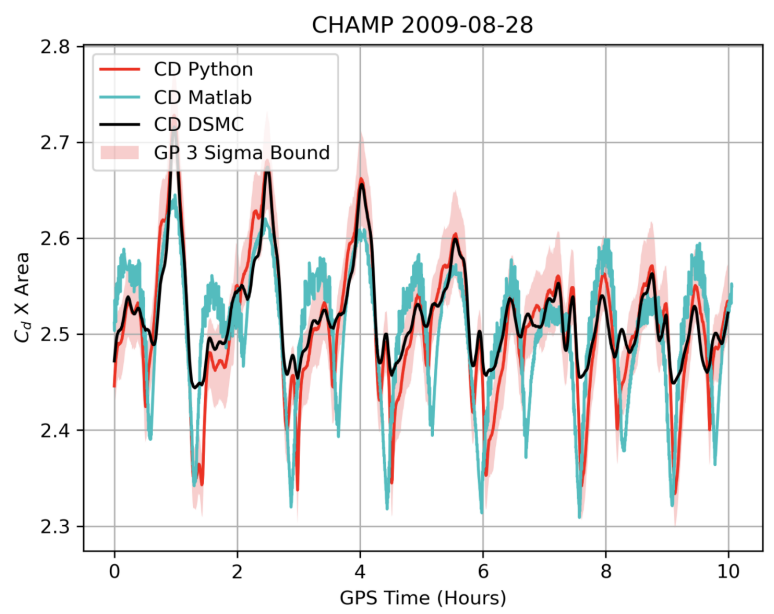

(b) CHAMP 2009 August 28, 10hr $C_{D} \times$ Area

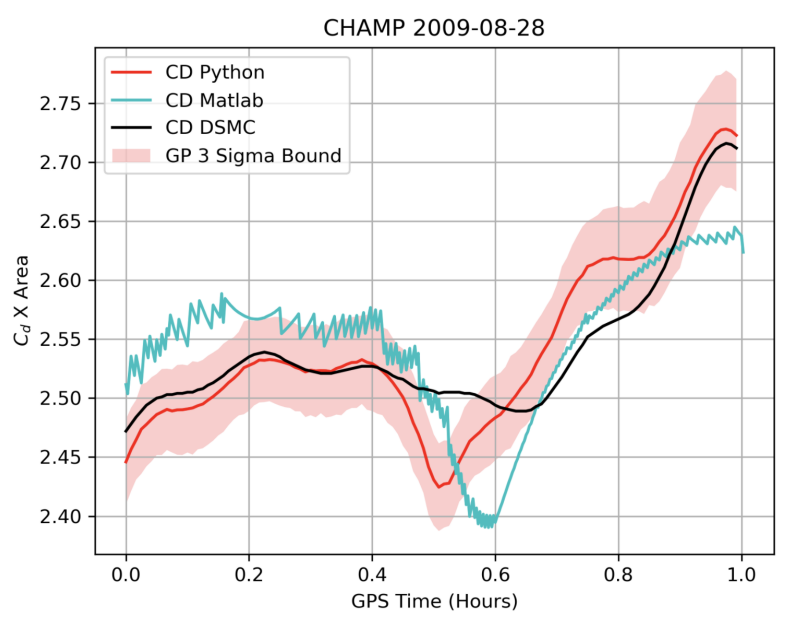

(c) CHAMP 2009 August 28, 1hr $C_{D} \times$ Area

Figure 4.15: Comparison of GPR versus known values 
Each of these plots shows the drag coefficient multiplied by the projected area of the satellite. Figure 4.15 consists of three plots:the first set of results is expanded over a twenty four hour period, while the other two figures are zoomed in versions with a ten hour period and one hour period, respectively. The "CD Python" is calculate using the WVU GPR and the "CD Matlab" uses the LANL GPR. "CD DSMC" is the drag coefficient generated from DSMC lookup tables. The results between the three data sets are very similar. The LANL GPR results do show a significant amount of noise, but for the most part falls within the bounds of uncertainty. The Python GPR results follow the DSMC trend quite closely. This shows that the Python GPR provides reasonable results.

\subsubsection{RUN TIME COMPARISON}

Most of the code that was added to the WVU RSM is comprised of entirely of new scripts or the same code from the LANL RSM. The new script's run times vary greatly across what the user input's into the software; however, the GPR was changed from the LANL's in-house code to Python's Scikit-learn package. This makes it worth noting the run time difference between the two scripts.

Comparison of the two codes is done with a model of a simple $1 \mathrm{~m}$ cube with 38 triangular facets. There are 1,000 simulations per species of gas comprising the test and training data. The Python GPR creation was completed in approximately 2 minutes. The MATLAB® GPR creation was able to do the same in approximately 420 minutes. 


\subsection{Documentation}

To further make the WVU RSM toolkit a more user friendly software, documentation was added for the user's benefit. The documentation is included with the download of the toolkit from GitHub and found underneath the DOCS folder. The documentation includes a full user manual complete with installation information as well as background, operation, troubleshooting notes, and analysis instructions. The author's information can be found within, as well as information for the other developers involved in the making of the toolkit. The toolkit is a GNU Publicly Licensed software with the appropriate information included. The WVU RSM software and documentation can be downloaded at the following GitHub Link: https://github.com/ASSISTLaboratory/WVU_ RSM_Suite/tree/main/WVU_RSM

Any further questions, comments, or concerns about the software can be emailed Dr. Piyush Mehta at piyush.mehta@mail.wvu.edu. The developers Logan Sheridan, Dr. Guillermo Avendano-France, and Dr. Smriti Paul can be emailed at the respective email addresses as well: pls0013@mix.wvu.edu, guavendanofranco@mail.wvu.edu, and smritinandan.paul@mail.wvu.edu

\section{CONClusion/Future WORK}

The main goal addressed was the need for a Response Surface Modeling software that uses Test Particle Monte Carlo to simulate drag on objects in the Free Molecular Flow regime. This software needed to be able to include objects with complex geometries such as a satellite with rotating solar panels. Another goal was to address the need for an open source software with explicit compilation 
and installation instructions that a new user is capable of operating with ease. The WVU RSM software package proved to meet all of these goals and provide accurate results.

\subsection{CONCLUSiON}

The software created has shown to be a diversified tool that allows a user to obtain desired results as well as perform individual tasks like rotating STLs and model evaluation. Furthermore, the software consists of automated checks in order to reassure the user that all the proper modules and packages are installed to operate. The software is also able to log developer information in case there ever is an issue.

Through testing, the WVU RSM was able to provide accurate results. The software showed how an STL could be rotated, combined with others, and checked for water-tightness in an efficient and accurate manner. The software extended this capability to create different configurations of an object and apply them to a Gaussian Process Regression model that could then be evaluated by a user with a desired configuration and freestream conditions. Lastly, the software is also capable of outputting the projected area of the satellite with said conditions with any number of components.

\subsection{FUTURE WORK}

While the WVU RSM has been turned in to a viable research tool, there are still some changes that could be made to increase the accuracy and ease-of-use for the software.

As stated, the WVU RSM uses a GPR modeling. This has the prime potential 
to be changed to a neural network. GPRs typically have a functionality form that is limited to the kernel they use. A neural network could allow for more flexibility in the analysis of data. The neural network also has the capability to handle larger data sets with more efficiency. While the GPR is still efficient, an neural network could extend capabilities to analyze more real world data.

The WVU RSM is still a software that requires a higher level knowledge of command scripting. With the significant increase in efficiency from the Python modeling there shows great promise to shift this type of analysis software to a desktop environment. The WVU RSM could potentially have its own graphical user interface with respective operating system installation. 


\section{Appendices}

\section{A. EXAMPLE OF WVU RSM TOOLKIT OPERATION WITH ROTATION}

This section is an example of a complete walk-through in calculating the drag on a simple Cube-Satellite (CubeSat) with rotating solar panels. This example begins after the installation and compilation of the code. The CubeSat being evaluated is shown in Figure A.1.

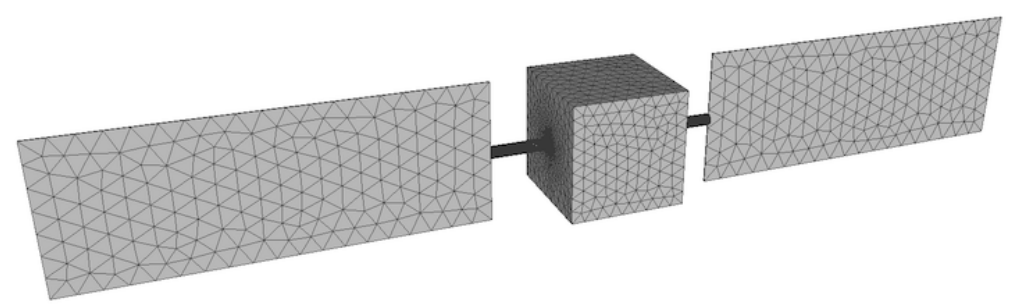

Figure A.1: Structure and Mesh of example CubeSat with Solar Panels

This satellite is made up of three distinct components; the left solar panel, the right solar panel, and the body. The body is compromised of the cube and the cylindrical arms on each side. Because the cylindrical arms are rounded, the mesh has be made much finer in these areas. The entire structure is made up of 10,025 facets in total. 


\section{A.1. Creating an STL File}

In order to create the actual structure of the object a CAD software is necessary. To create this satellite, DS Solidworks ${ }^{\circledR}$ was used. Any CAD software may be used to accomplish this task, but there are some notable steps to making sure the final structure is comprised of each component correctly.

The coordinate frame each component is made in is vital to making sure the final structure is built correctly. For the case of the satellite being built here, the center of the cube is $(0,0,0)$. To reiterate, the orientation of the reference frame is important for simulation. For this example, the satellite is symmetrical so the orientation is only important for the consideration of what direction the solar panels are rotating.

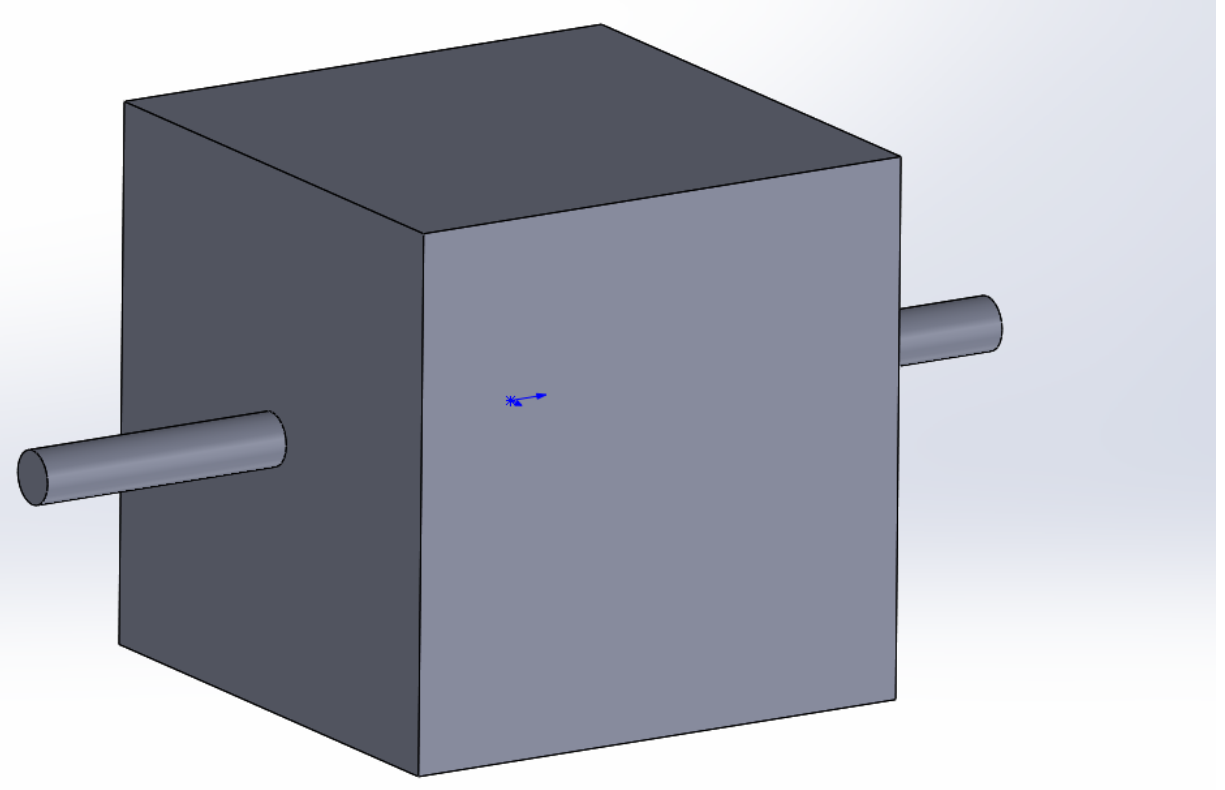

Figure A.2: Body Component of Example CubeSat 


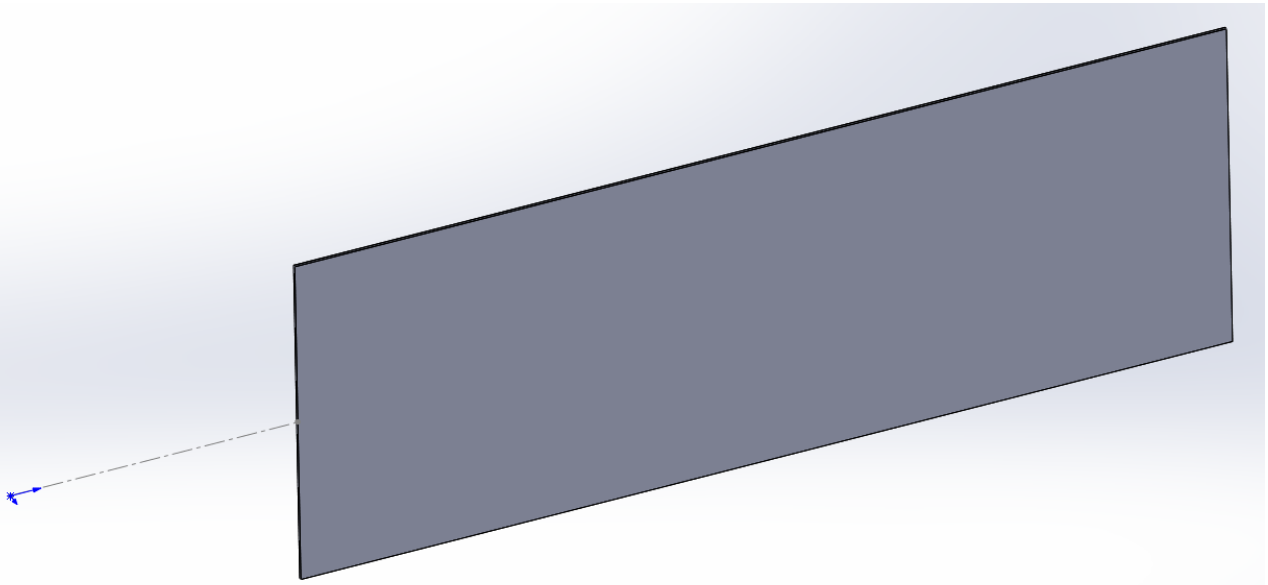

Figure A.3: Left Solar Panel Component of Example CubeSat

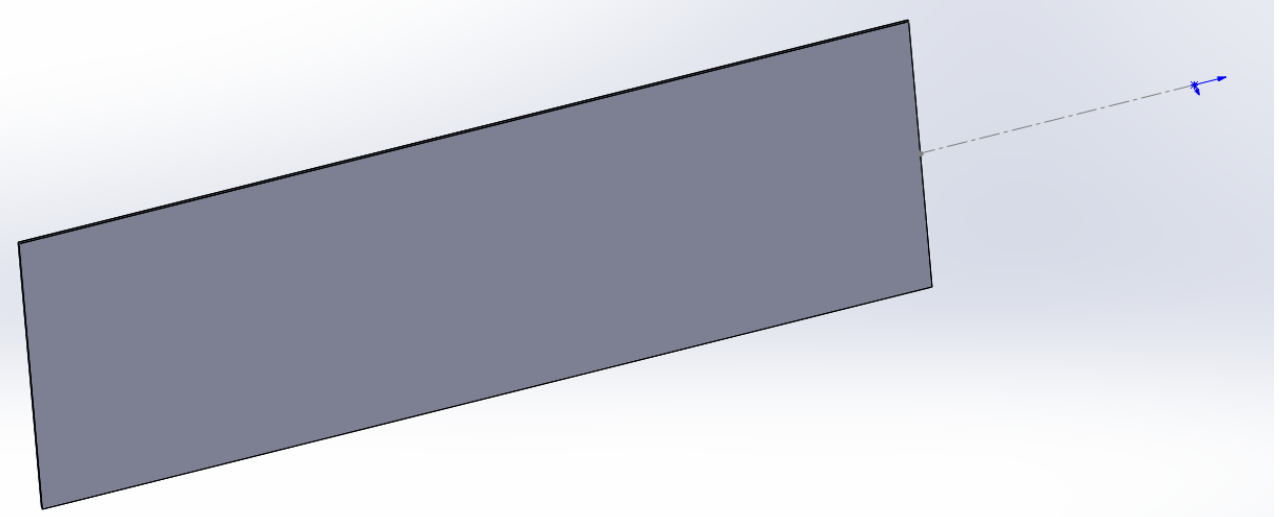

Figure A.4: Right Solar Panel Component of Example CubeSat

Note that each of the solar panels is 1 meter from the center, as shown by the dotted line. Since the body is a cube that is $1 \times 1 \times 1 \mathrm{~m}$ and each arm is $0.5 \mathrm{~m}$ long, the left panel connects at the coordinate points $(0,1000,0)$ and the right panel connects at the coordinates $(0,-1000,0)$. The scale of these coordinate points 
suggests the satellite is defined in millimeters although ASCII STL files do not contain unit information. When exporting components from CAD software, some CADs will translate the component so that it is centered to $(0,0,0)$. It is important a user makes sure that the parts remain in the correct reference frame. Most CADs allow the user to turn off translation.

After the structure has been developed the body must be meshed. Most CAD softwares, such as DS Solidworks ${ }^{\circledR}$, allow the user to export directly to an ASCII STL file. The user should be wary of this as the STLs created from CAD are not necessarily high fidelity meshes. To be sure of an even and controlled mesh, the user should use a meshing software. The open source software GMSH was used in the example. An example of the mesh generated for the body of the satellite is shown below.

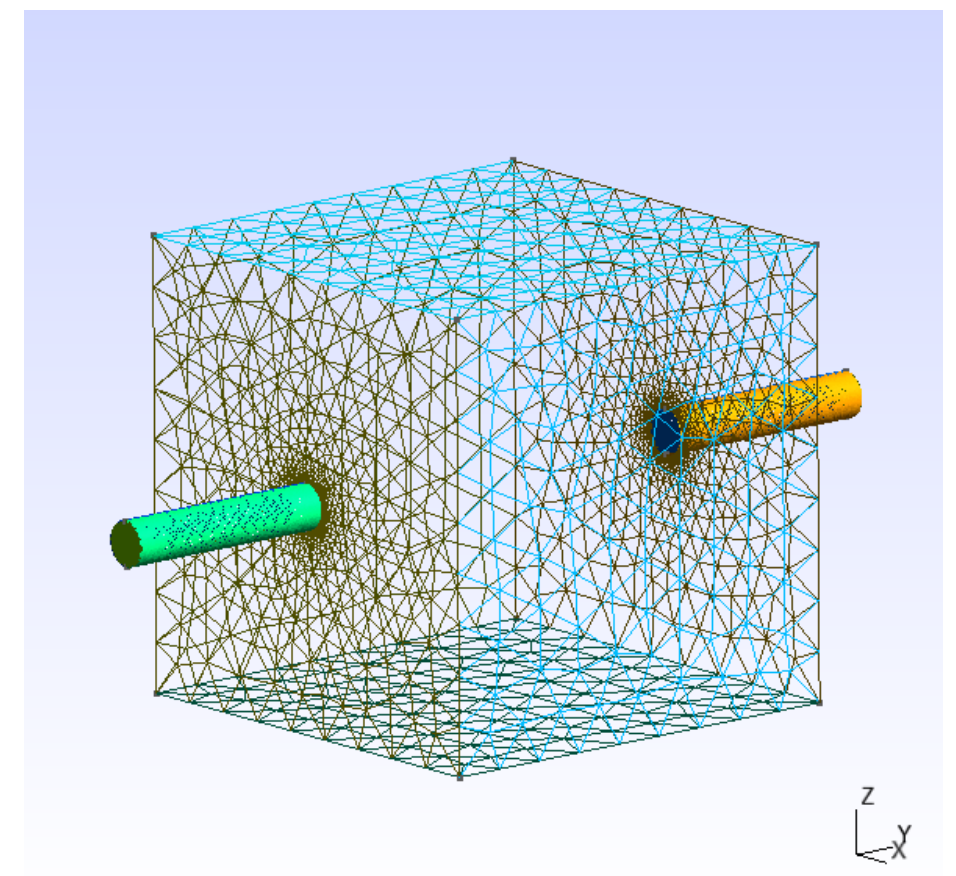

Figure A.5: Mesh for Body Component of Example CubeSat, GMSH 


\section{A.2. EXAMPLE InPUTS}

After creation and meshing of the components, the user can perform the setup for the simulations. First, they must navigate to the Inputs directory and place the components that were created into the STL_Files folder. Secondly, they must set up the Simulation.json file to the desired ranges. For this example, the Simulation.json is shown below. The file shows that 1000 ensemble points will be used with 200 processors. The sampling for yaw and pitch are \pm 10 degrees, the component rotation is 0:0 degrees for the body and 0:20 degrees for each panel.

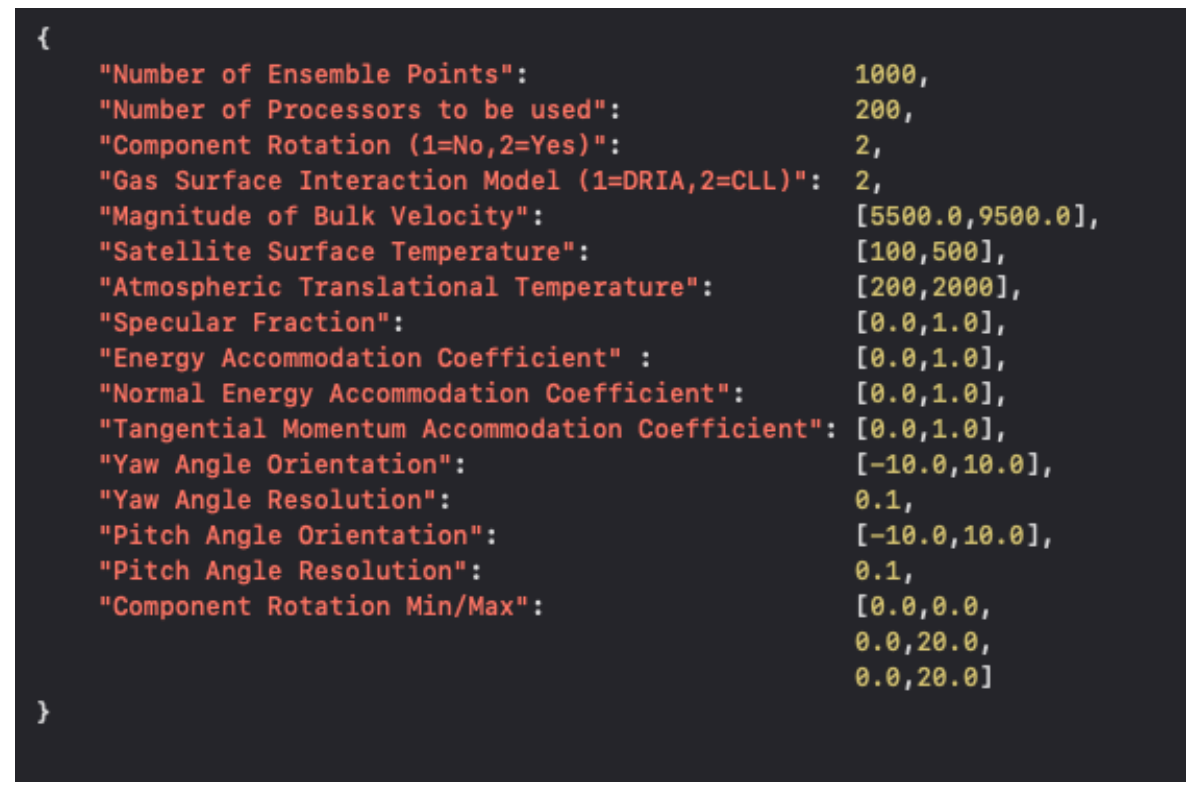

Figure A.6: Simulation Input for Example

Finally, the user needs to define the rotation inputs. Recalling there are three input files for rotation, the files are shown in Figure 4.8. 


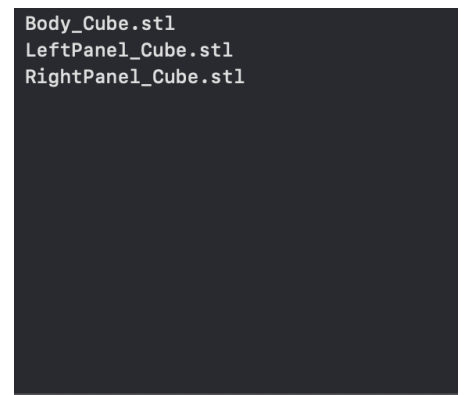

(a) parent.txt

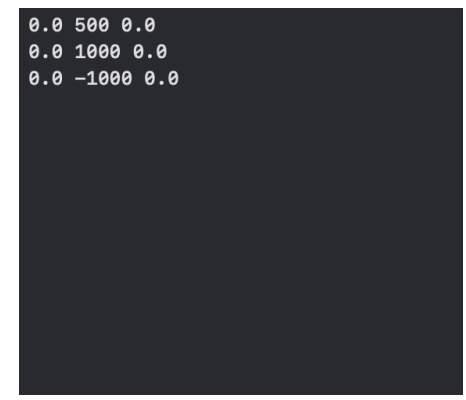

(b) hinge_points 1.txt

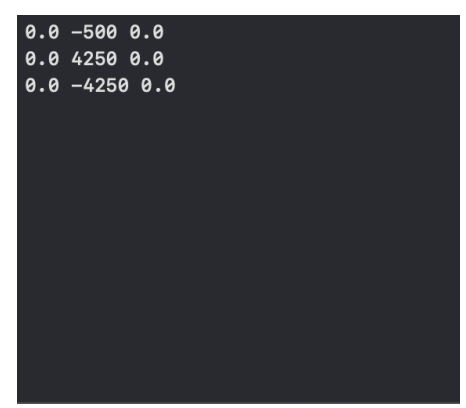

(c) hinge_points $2 . t x t$

Figure A.7: Example Rotation Inputs

The user should recall that the hinge points follow the right hand rule. An illustration of the hinge point setup is shown in Figure 4.9.

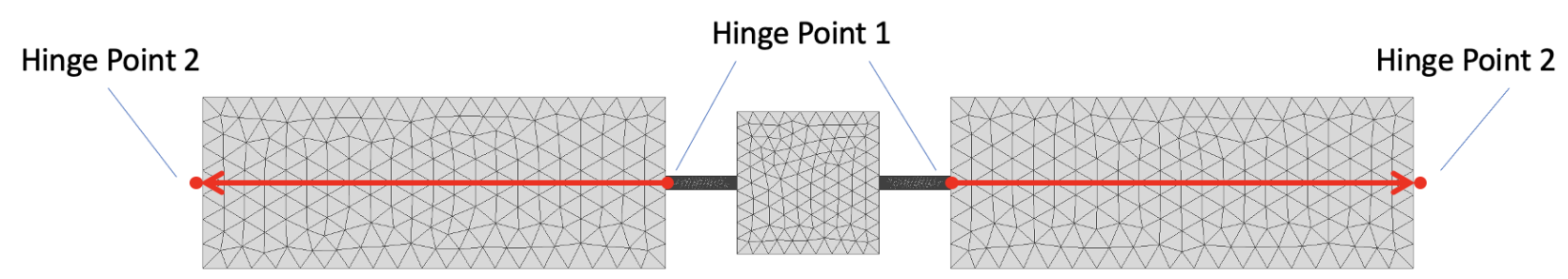

Figure A.8: Simulation Input for Example

For this example, the two inner joints were chosen as the first hinge point of each vector and the outer points are the second. This is to illustrate the right hand rule. In the input file 0:20 was chosen for both, this will result in the 
solar panels rotating in opposite directions. The figure below shows the positive direction of rotation for each panel.

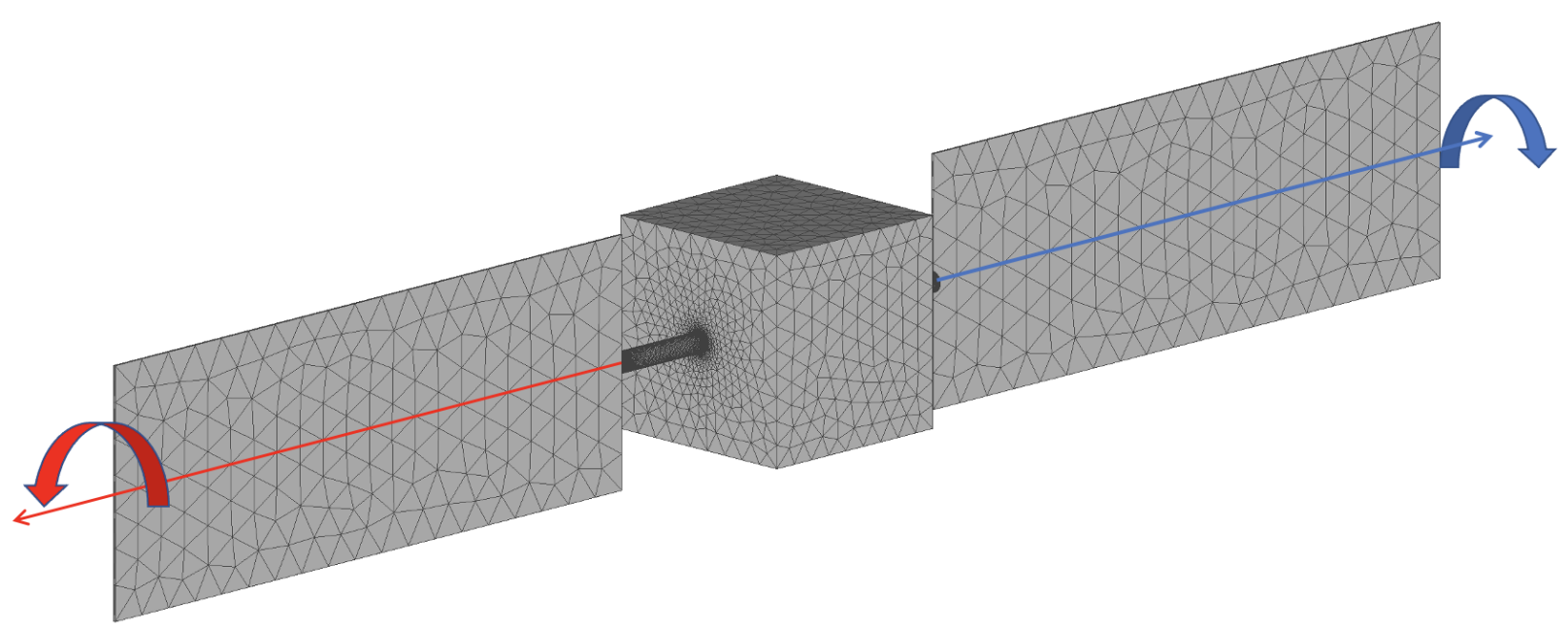

Figure A.9: Rotation Direction for Example

\section{A.3. EXAMPLE EXECUTION}

For this simulation the GCC C compilers were used along with Intel Python modules. The code is executed using the following command:

\$./RSM_TPMC/rsm_run_script.py --tpm I RSM_TPMC/tpm/build/src/tpm

Once executed the the RSM logo and some other miscellaneous print statements will output. An example of the "Watertight" statement can also be seen in the figure below. When the rotation software creates the STL files for the TPMC simulation they are named with an integer; e.g. Satellite0.stl. The function that checks water-tightness instead outputs the angle deflections, in order of each 
component, attached to the name; e.g. Satellite_0.0_-5.0_10.235.stl. This can

be useful if the user is having a certain rotation that is throwing an error.

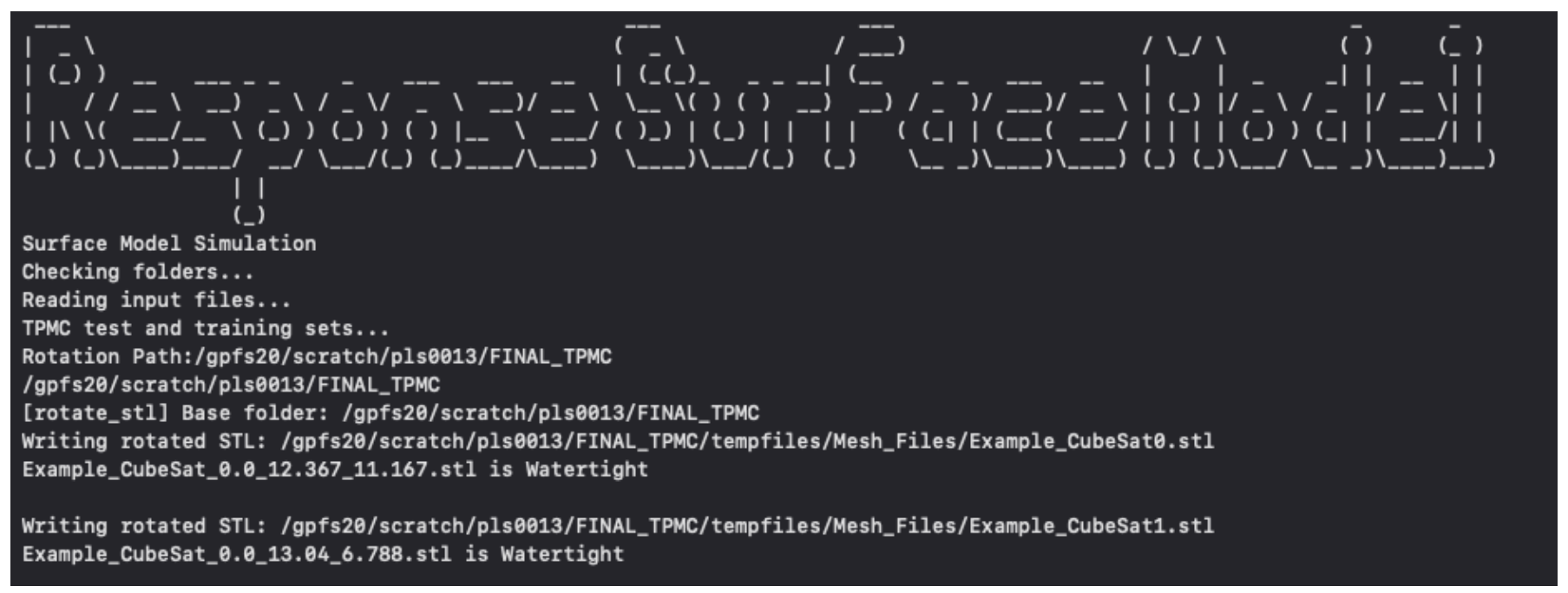

Figure A.10: RSM Logo and Initial Output

\section{A.4. EXAMPLE OUTPUTS}

Once the RSM has finished running, the aforementioned outputs will have automatically been created. The user will need to move the regression models in the Output folder to the Model_Evaluation_Inputs folder.

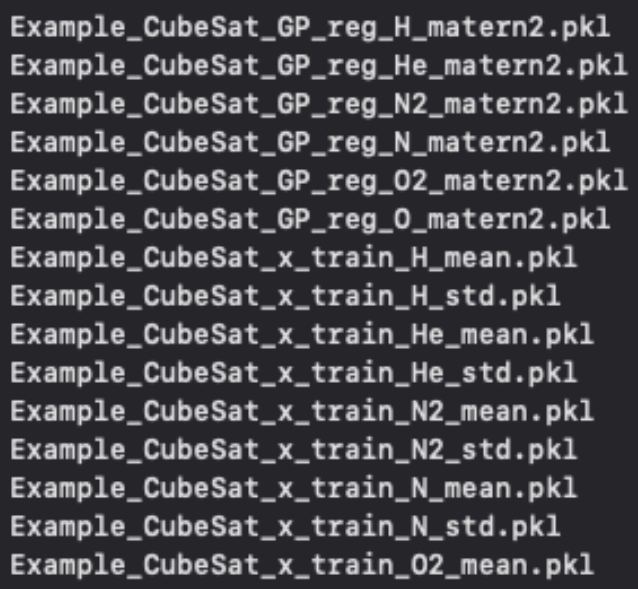

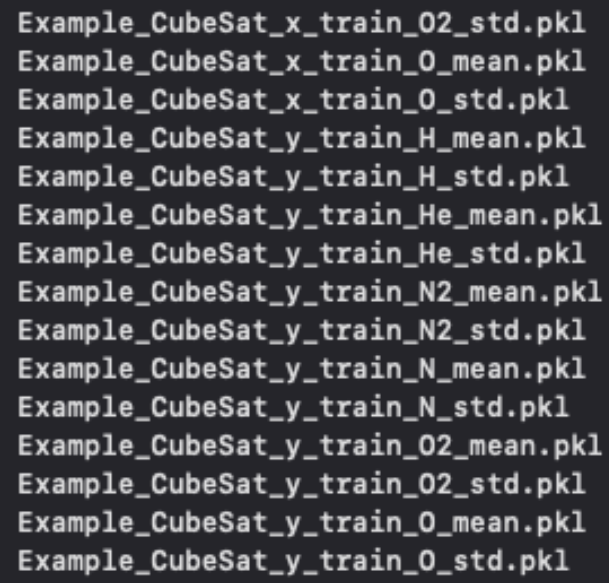

Figure A.11: Thirty Files for the Example Regression Model 


\section{A.5. Example Model Evaluation}

At this point in the execution of the RSM, the model is ready to be evaluated. The CSV file needs to be set up with the desired evaluation points and the JSON file needs to reflect the proper name and values. Headers in the CSV example are stacked for visual purposes only. The headers should be in a single row in the actual file.

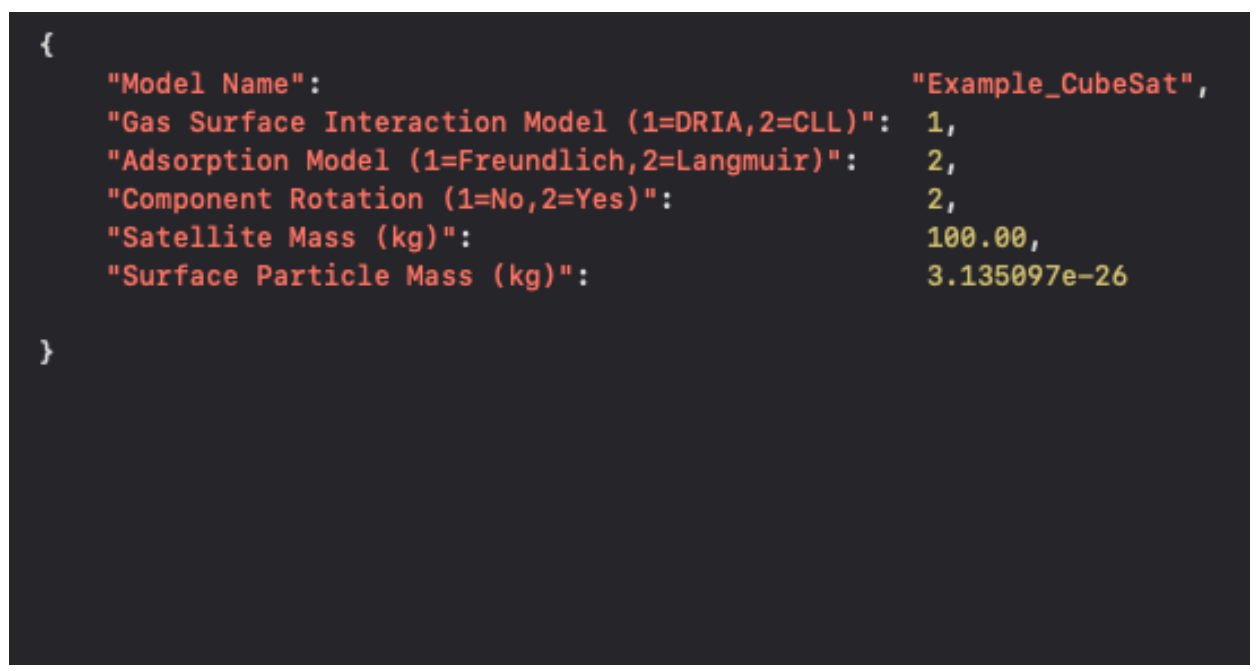

Figure A.12: Example Model_Evaluation.json file

\begin{tabular}{|c|c|c|c|c|c|c|c|c|c|c|c|c|}
\hline Velocity & $\begin{array}{l}\text { Temperature T } \\
\text { (K) }\end{array}$ & $\begin{array}{l}\text { tmospheric } \\
\text { emperature } \\
\text { K) }\end{array}$ & aw & Pitch & $\begin{array}{l}\text { Density } \\
\left(\# / m^{\wedge} 3\right)\end{array}$ & $\begin{array}{l}\text { Mole } \\
\text { Fraction: } \mathrm{H}\end{array}$ & $\begin{array}{l}\text { Mole } \\
\text { Fraction: He }\end{array}$ & $\begin{array}{l}\text { Mole } \\
\text { Fraction: } N\end{array}$ & $\begin{array}{l}\text { Mole } \\
\text { Fraction: N2 }\end{array}$ & $\begin{array}{l}\text { Mole } \\
\text { Fraction: O }\end{array}$ & $\begin{array}{l}\text { Mole } \\
\text { Fraction: } 02\end{array}$ & Component Rotations...: \\
\hline 7665.73083 & 300 & 1196.0596 & 0.049679 & -0.004151 & $3.44 E+14$ & 0.015457 & 0.889884 & 0.072458 & 0.001046 & 0.000151 & 0.021004 & 0 \\
\hline 7665.90411 & 300 & 1195.1422 & 0.050152 & -0.00426 & $3.44 E+14$ & 0.015585 & 0.890482 & 0.071902 & 0.001034 & 0.000152 & 0.020846 & 0 \\
\hline 7666.07134 & 300 & 1163.8846 & 0.050376 & -0.004166 & $3.19 E+14$ & 0.016939 & 0.894891 & 0.067694 & 0.000952 & 0.000183 & 0.01934 & 0 \\
\hline 7666.23274 & 300 & 1163.1406 & 0.050544 & -0.004006 & $3.19 E+14$ & 0.017074 & 0.895354 & 0.06723 & 0.000943 & 0.000184 & 0.019215 & 0 \\
\hline 7666.38841 & 300 & 1162.4384 & 0.050843 & -0.003989 & $3.20 E+14$ & 0.017211 & 0.895796 & 0.066782 & 0.000934 & 0.000185 & 0.019093 & 0 \\
\hline 7666.53832 & 300 & 1161.7753 & 0.051026 & -0.00384 & $3.20 \mathrm{E}+14$ & 0.01735 & 0.896218 & 0.066348 & 0.000925 & 0.000185 & 0.018974 & 0 \\
\hline 7666.68231 & 300 & 1161.1489 & 0.05144 & -0.003941 & $3.20 E+14$ & 0.017491 & 0.896619 & 0.065928 & 0.000917 & 0.000186 & 0.018859 & 0 \\
\hline 7666.82019 & 300 & 1160.5573 & 0.051785 & -0.003992 & $3.20 E+14$ & 0.017634 & 0.897001 & 0.065522 & 0.000909 & 0.000187 & 0.018748 & 0 \\
\hline 7666.95195 & 300 & 1159.9973 & 0.052181 & -0.004078 & $3.20 \mathrm{E}+14$ & 0.01778 & 0.897365 & 0.065127 & 0.000901 & 0.000187 & 0.01864 & 0 \\
\hline 7667.07754 & 300 & 1159.4667 & 0.052597 & -0.00421 & $3.20 E+14$ & 0.017928 & 0.89771 & 0.064744 & 0.000894 & 0.000188 & 0.018535 & 0 \\
\hline 7667.19687 & 300 & 1158.9626 & 0.05296 & -0.004292 & $3.20 E+14$ & 0.018079 & 0.898038 & 0.064373 & 0.000887 & 0.000189 & 0.018434 & 0 \\
\hline
\end{tabular}

Figure A.13: Example CSV file

Eleven points were used to evaluate the model, therefore there should be eleven projected areas output and eleven drag coefficients. Navigating to the 
Outputs folder, there is now a file for each. In the projected area file below, it can be seen that each area is on the order of $10^{6}$. This is because the STL file used values that are in millimeters.

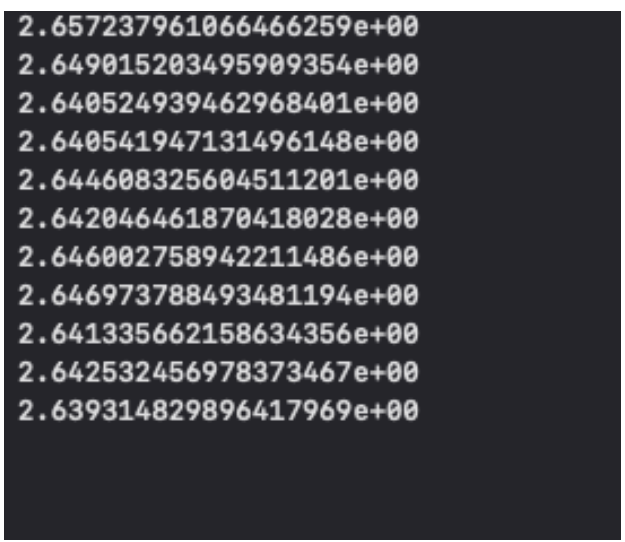

(a) $C_{D}$ Results

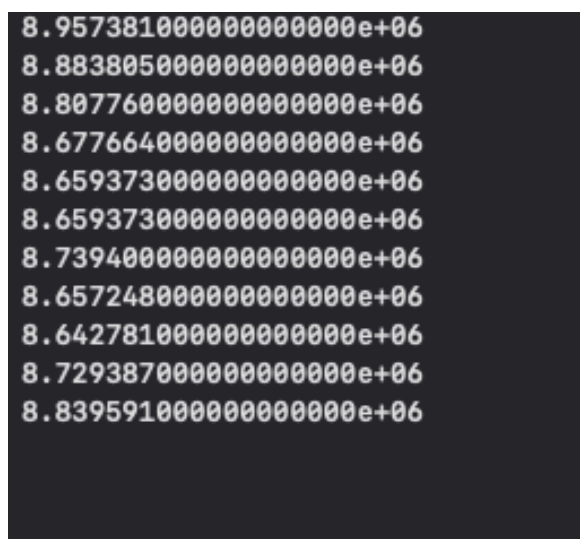

(b) Area Results

Figure A.14: Example Results 


\section{B. Code Resources}

\section{B.1. CODE}

The Los Alamos National Laboratory Response Surface Modeling code can be found at:

https://github.com/AndrewCWalker/rsm_tool_suite

Reference for this code can be found under the following paper:

Mehta, P. M., Walker, A., Lawrence, E., Linares, R., Higdon, D., and Koller, J. (2014). Modeling Satellite Drag Coefficients with Response Surfaces. Advances in Space Research, 54(8), 1590-1607. doi:10.1016/j.asr.2014.06.033

The West Virginia University Response Surface Modeling code can be found at:

https://github.com/ASSISTLaboratory/WVU_RSM_Suite

\section{B.2. DeVELOPERS}

Logan Sheridan, Lead Developer, Author

pls0013@mix.wvu.edu

Dr. Guillermo Avendano-Franco, Cluster Specialist, AutoTools

guavendanofranco@mail.wvu.edu

Dr. Smriti Paul, GPR Creation

smritinandan.paul@mail.wvu.edu

Dr. Piyush M. Mehta, Advisor, WVU Assistant Professor

piyush.mehta@mail.wvu.edu 


\section{REFERENCES}

[1] Bird, G. A.; Approach to Translational Equilibrium in a Rigid Sphere Gas, Physics of Fluids, Vol. 6, 1963, pp. 1518.

[2] Bird, G. A.; Molecular Gas Dynamics and the Direct Simulation of Gas Flows Oxford Science Publication, New York, 1994.

[3] Cercignani, C., and Lampis, M.; Kinetic Models for Gas-Surface Interactions, Transport Theory and Statistical Physics, Vol. 1, No. 2, 1971, pp. $101-114$

[4] CNBC; Why in the next decade companies will launch thousands more satellites than in all of history https://www.cnbc.com/2019/12/14/spacexoneweb-and-amazon-to-launch-thousands-more-satellites-in-2020s.html

[5] Davis, D.H.; Monte Carlo Calculation of Molecular Flow Rates Through a Cylindrical Elbow and Pipes of Other Shapes. Journal of Applied Physics, Vol. 31, 1961, pp. 1169-1176.

[6] Enrico Camporeale and Algo Carè, Estimation of Accurate and Calibrated Uncertainties in Deterministic models 2020

[7] Foo, K. Y., \& Hameed, B. H.; Insights into the modeling of adsorption isotherm systems. Chemical Engineering Journal, 156(1), 2-10. doi:10.1016/j.cej.2009.09.013

[8] Freundlich, H.; Über die Adsorption in Lösungen. Zeitschrift Für Physikalische Chemie, 1907, 57U(1). doi:10.1515/zpch-1907-5723

[9] Gaussian Process https://scikit-learn.org/stable/modules/gaussian_process.html 
[10] Gemma J. Anderson and Jim A. Gaffney and Brian K. Spears and PeerTimo Bremer and Rushil Anirudh and Jayaraman J. Thiagarajan, Meaningful uncertainties from deep neural network surrogates of large-scale numerical simulations, 2020

[11] Goodman, F. O., and Wachmann, H. Y.; Formula for Thermal Accommodation Coefficient Massachusetts Inst. of Technology, Fluid Dynamics Lab. Rept. 66-1, Cambridge, MA, 1966.

[12] Goodman, F. O.; Thermal Accommodation, Progress in Surface Science, Vol. 5, No. 3, 1974, pp. 261-375.

[13] Günther, March , Visser, Pieter N. A. M., Doornbos, Eelco; CHAMP, GRACE, GOCE and Swarm density and wind characterization with improved gas-surface interactions modelling 42nd COSPAR Scientific Assembly. Held 14-22 July 2018, in Pasadena, California, USA, Abstract id. C1.5-20-18.

[14] Hedin, A. E., Hinton, B. B., and Schmitt, G. A.; Role of Gas-Surface Interactions in the Reduction of Ogo 6 Neutral Particle Mass Spectrometer Data, Journal of Geophysical Research, Vol. 78, No. 22, 1973, pp. 4651-4668. doi:10.1029/JA078i022p04651

[15] Hinchen, J. J., and Foley, W. M.; Scattering of Molecular Beams by Metallic Surfaces, Proceedings of the 4th International Symposium on Rarefied Gas Dynamics, Academic Press, New York, 1966, pp. 505- 512.

[16] Hurlbut, F.; On the Molecular Interactions Between Gases and Solids, Univ. of California TR-HE-150-208, Berkeley, CA, 1962. 
[17] Hurlbut, F.; Studies of Molecular Scattering at the Solid Surface, Journal of Applied Physics, Vol. 28, No. 8, 1957, p. 844-850. doi:10.1063/1.1722872

[18] Kleyn, A.; Molecular Beam Scattering at Metal Surfaces, Surface Dynamics Elsevier, New York, 2003, pp. 79-108.

[19] Knudsen, M.; Das Cosinusgesetz in der Kinetischen Gastheorie, Annalen der Physik, Vol. 353, No. 24, 1915, pp. 1113-1121.

[20] Langmuir, I.; THE CONSTITUTION AND FUNDAMENTAL PROPERTIES OF SOLIDS AND LIQUIDS. PART I. SOLIDS. Journal of the American Chemical Society, 38(11), 2221-2295, 1916, doi:10.1021/ja02268a002

[21] Lord, R.; Application of the Cercignani-Lampis Scattering Kernel to Direct Simulation Monte Carlo Calculations, Proceedings of the 17th International Symposium on Rarefied Gas Dynamics, Wiley-VCH Verlag GmbH, Weinheim, Germany, 1990, pp. 1427-1433.

[22] Mehta, P. M., Walker, A., Lawrence, E., Linares, R., Higdon, D., \& Koller, J.; Modeling satellite drag coefficients with response surfaces. Advances in Space Research, 54(8), 1590-1607, 2014, doi:10.1016/j.asr.2014.06.033

[23] Moe, K., and Moe, M. M.; The Effect of Adsorption on Densities Measured by Orbiting Pressure Gauges, Planetary and Space Science, Vol. 15, No. 8, 1967, pp. 1329-1332. doi:10.1016/0032-0633(67)90186-9

[24] Moe, K., Moe, M. M., \& Wallace, S. D.; Improved Satellite Drag Coefficient Calculations from Orbital Measurements of Energy Accommodation. Journal of Spacecraft and Rockets, 35(3), 266-272, 1998, doi:10.2514/2.3350 
[25] Moe, K., Moe, M. M., and Doornbos, E.; Outstanding Issues related to Thermospheric Measurements and Modeling, http://spaceweather.usu.edu/files/ uploads/PDF/Outstanding_Issues.pdf, 2012.

[26] Moe, M. M., Moe, K.; The Roles of Kinetic Theory and Gas-Surface Interactions in Measurements of Upper-Atmospheric Density, Planetary and Space Science, Vol. 17, No. 5, 1969, pp. 917-922

[27] NASA; Space Debris and Human Spacecraft https://www.nasa.gov/mission_pages/station/news/orbital_debris.html

[28] Pilinski, M. D., Argrow, B. M., and Palo, S. E.; Drag Coefficientsof Satellites with Concave Geometries: Comparing Models and Observations, Journal of Spacecraft and Rockets, Vol. 48, No. 2, 2011, pp. 312-325. doi:10.2514/1.50915

[29] Pilinski, M. D., Argrow, B. M., and Palo, S. E.; Semi-Empirical Model for Satellite Energy-Accommodation Coefficients, Journal of Spacecraft and Rockets, Vol. 47, No. 6, 2010, pp. 951-956.

[30] Pilinski, M. D., Argrow, B. M., Palo, S. E., \& Bowman, B. R.; SemiEmpirical Satellite Accommodation Model for Spherical and Randomly Tumbling Objects Journal of Spacecraft and Rockets, 50(3), 556-571, 2013, doi:10.2514/1.a32348

[31] C. E. Rasmussen, K. I. Williams, Gaussian Processes for Machine Learning. Massachusetts Institute of Technology: MIT Press, 2006.

[32] Sacks, J., Welch, W.J., Mitchell, T.J., Wynn, H.P.; Design and analysis of computer experiments. Stat. Sci. 4 (4), 409-435, 1989 
[33] Schaaf, S. A., and Chambre, P. L.; Flow of Rarefied Gases, Princeton University Press, Princeton, NJ, 1961.

[34] L.H. Sentman; Free molecule flow theory and its application to the determination of aerodynamic forces Oct. 1961, Lockheed Missiles \& Space Co., Sunny Vale, California

[35] Space News; SpaceX launches fifth batch of Starlink satellites, misses booster landing https://spacenews.com/spacex-launches-fifth-batch-ofstarlink-satellites-misses-booster-landing/

[36] Tancred, James A.; ROTATESTL: A MATLAB ROTATION ALGORITHM FOR THE ANALYSIS OF COMPUTATIONAL MESHES IN STEREOLITHOGRAPHY FILE FORMAT Air Force Research Laboratory, Aerospace Systems Directorate, Wright-Patterson Air Force Base, Air Force Materiel Command, United States Air Force, 2012.

[37] Walker, A., Mehta, P., \& Koller, J.; Drag Coefficient Model Using the Cercignani-Lampis-Lord Gas-Surface Interaction Model. Journal of Spacecraft and Rockets, 51(5), 1544-1563, 2014, doi:10.2514/1.a32677 doi: $10.2514 / 1.49330$

[38] Walker, A., Mehta, P., \& Koller, J.; Different Implementations of Diffuse Reflection with Incomplete Accommodation for Drag Coefficient Modeling. Journal of Spacecraft and Rockets, 51(5), 1522-1532, 2014, doi:10.2514/1.a32668 